\author{
Aus der Abteilung Unfallchirurgie, \\ Plastische und Wiederherstellungschirurgie \\ (Prof. Dr. med. K. M. Stürmer) \\ im Zentrum Chirurgie \\ der Medizinischen Fakultät der Universität Göttingen
}

\title{
Klinische und radiologische Ergebnisse nach operativ versorgter Patellafraktur
}

\author{
INAUGURAL-DISSERTATION \\ zur Erlangung des Doktorgrades \\ der Medizinischen Fakultät \\ der Georg-August-Universität zu Göttingen \\ vorgelegt von \\ Cyrus Behzadi aus \\ Eschwege/Hessen
}

Göttingen 2013 
Dekan: Prof. Dr. rer. nat. H.K. Kroemer

I. Berichterstatter: Prof. Dr. med. K.-H. Frosch

II. Berichterstatter: Prof. Dr. med. C. Engelke

III. Berichterstatter: Prof. Dr. med. dent. R. Mausberg

Tag der mündlichen Prüfung: 04.11.2013 


\section{Inhaltsverzeichnis}

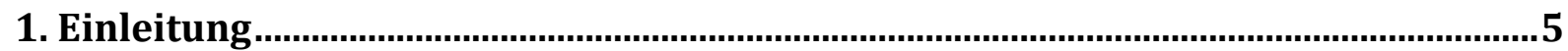

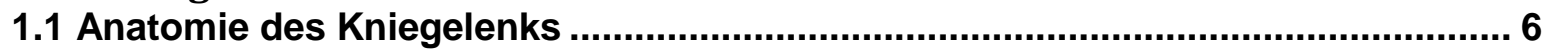

1.2 Anatomie und Funktion der Patella ......................................................................... 7

1.3 Klassifikation der Patellafrakturen ................................................................. 10

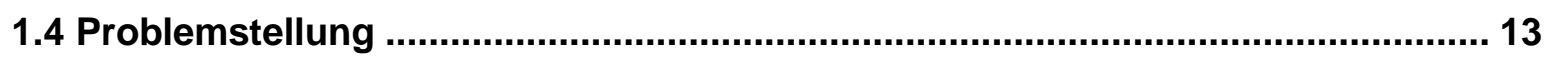

1.5 Zielsetzung der Studie......................................................................................... 19

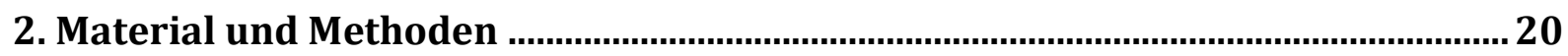

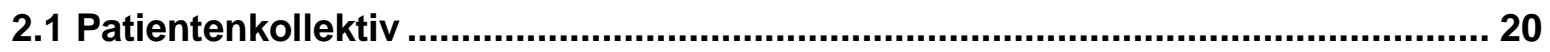

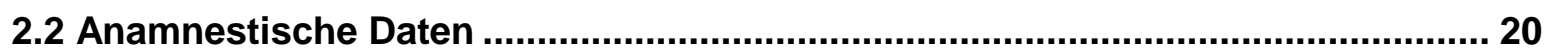

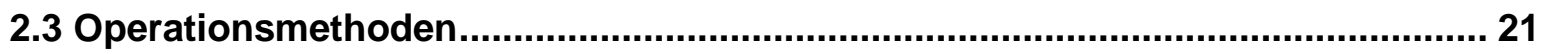

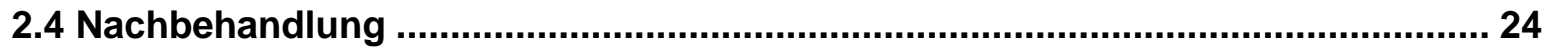

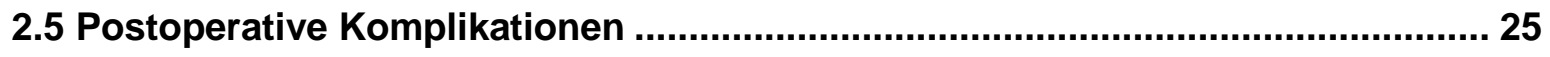

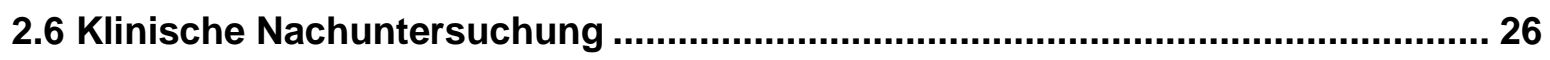

2.6.1 VASS (Visuell numerische Analogskala Schmerz)...................................................................26

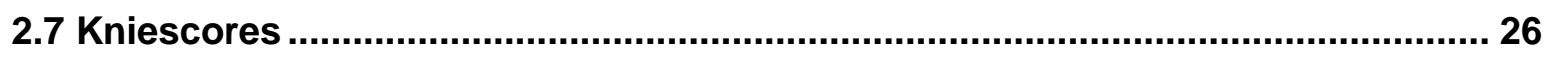

2.7.1 AKSS (American Knee Society Score) ..................................................................................26

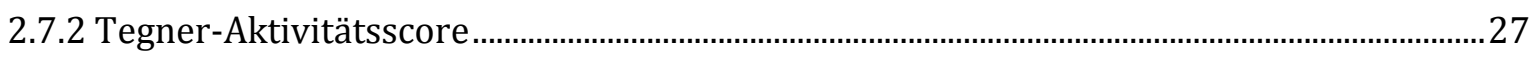

2.7.3 WOMAC-Score (Western Ontario \& McMaster Universities Osteoarthritis Index)............27

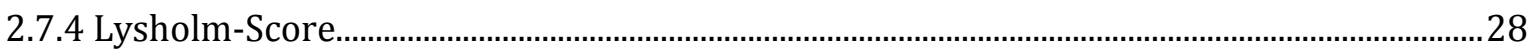

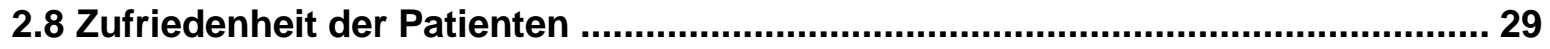

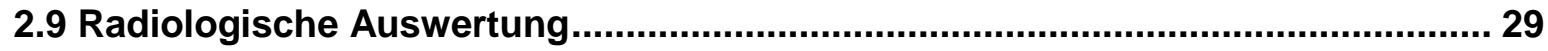

2.10 Histologische Untersuchung der Patella ................................................. 31

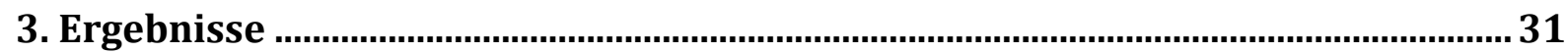

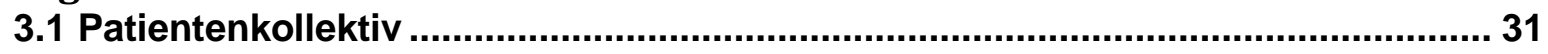

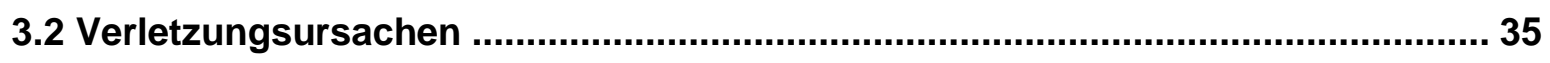

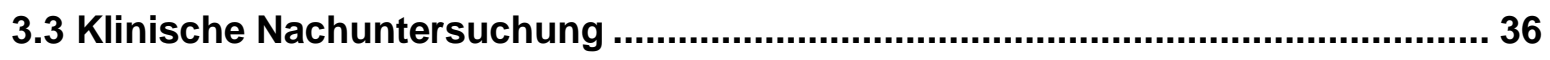

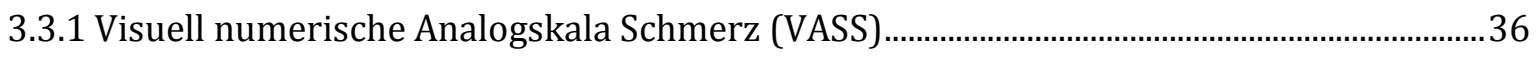

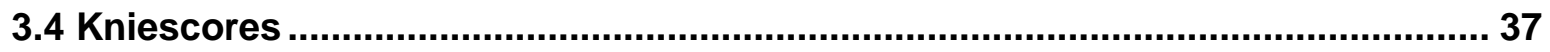

3.4.1 American Knee Society Score (AKSS) ..........................................................................................37 


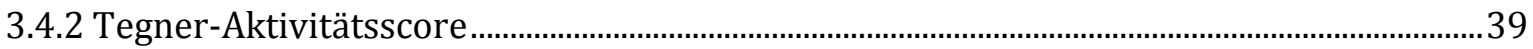

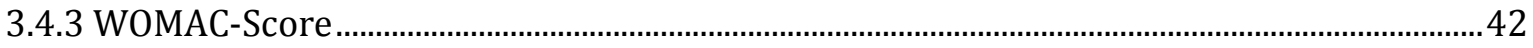

3.4.4 Lysholm-Score

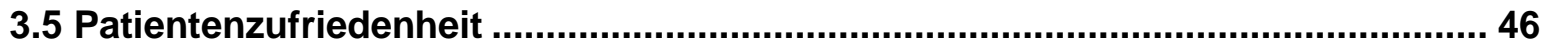

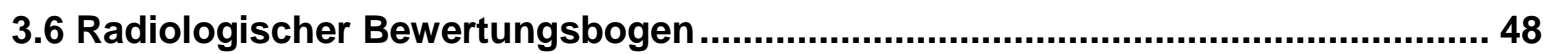

3.6.1 Visuell numerische Analogskala Schmerz .............................................................................. 48

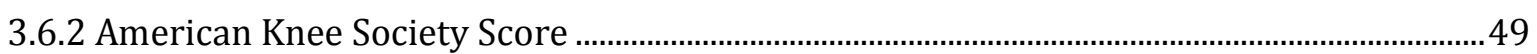

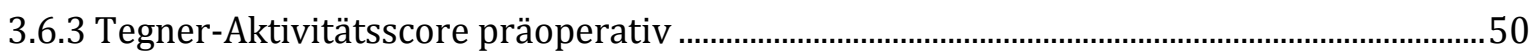

3.6.4 Tegner-Aktivitätsscore postoperativ.........................................................................................51

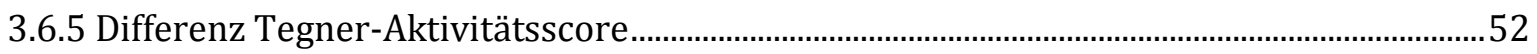

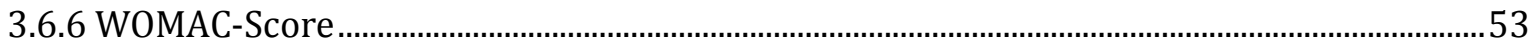

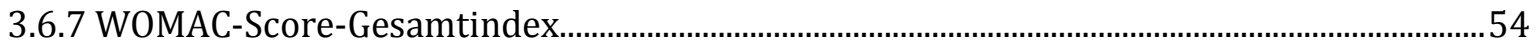

3.6.8 Lysholm-Score

3.6.9 Patientenzufriedenheit................................................................................................... 56

3.7 Vergleich der Ergebnisse von Patienten mit hyperdensen Arealen gegenüber

Patienten ohne Auffälligkeiten .......................................................................... 57

3.7.1 Visuell numerische Analogskala Schmerz (VASS)...................................................................

3.7.2 American Knee Society Score (AKSS) .......................................................................................5

3.7.3 Tegner-Aktivitätsscore (präoperativ)......................................................................................59

3.7.4 Tegner-Aktivitätsscore (postoperativ) ...................................................................................6

3.7.5 Differenz im Tegner-Aktivitätsscore (prä- zu postoperativ)....................................................62

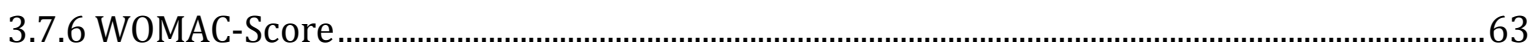

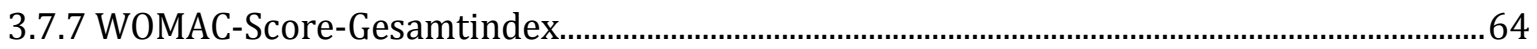

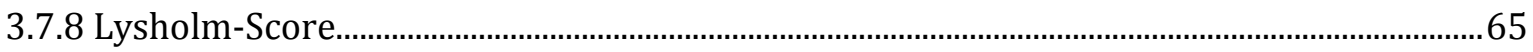

3.7.9 Patientenzufriedenheit..............................................................................................................66

3.8 Histologische Untersuchung der Patella ......................................................................................67

4. Diskussion

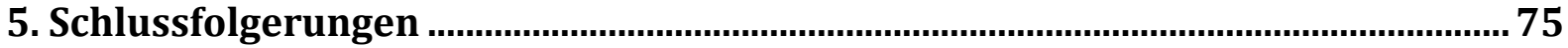

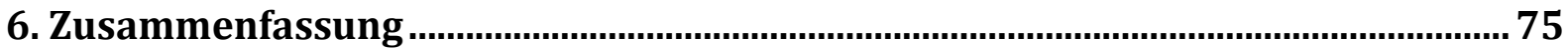

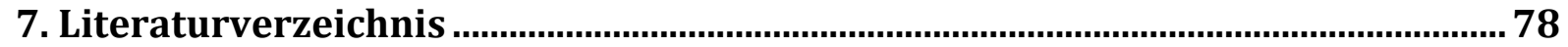

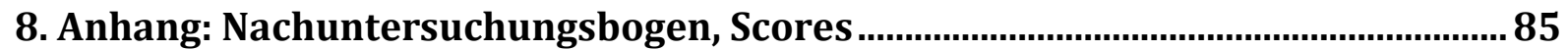

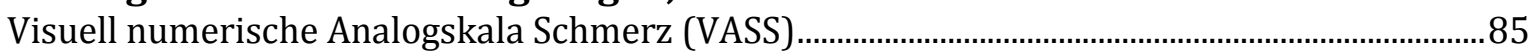

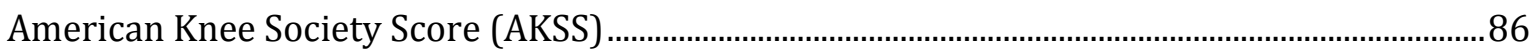

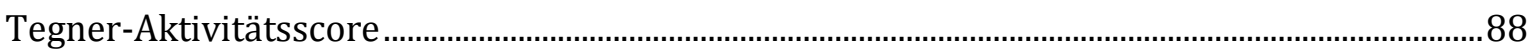

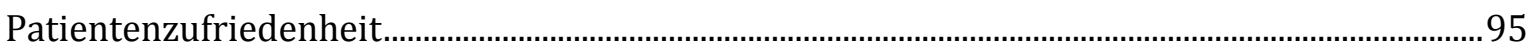




\section{Einleitung}

Patellafrakturen entsprechen ca. 0,5 - 1,5\% aller Frakturen des menschlichen Skeletts (Galla und Lobenhoffer 2005). Patellafrakturen treten vorwiegend als Folge direkter Krafteinwirkungen, zum Beispiel im Rahmen von Verkehrsunfällen („Dashboard-Injury“), oder durch einen Sturz auf das gebeugte Kniegelenk auf. Dies wird durch ihre exponierte Lage direkt unter der Haut erklärt (Böstman et al. 1983).
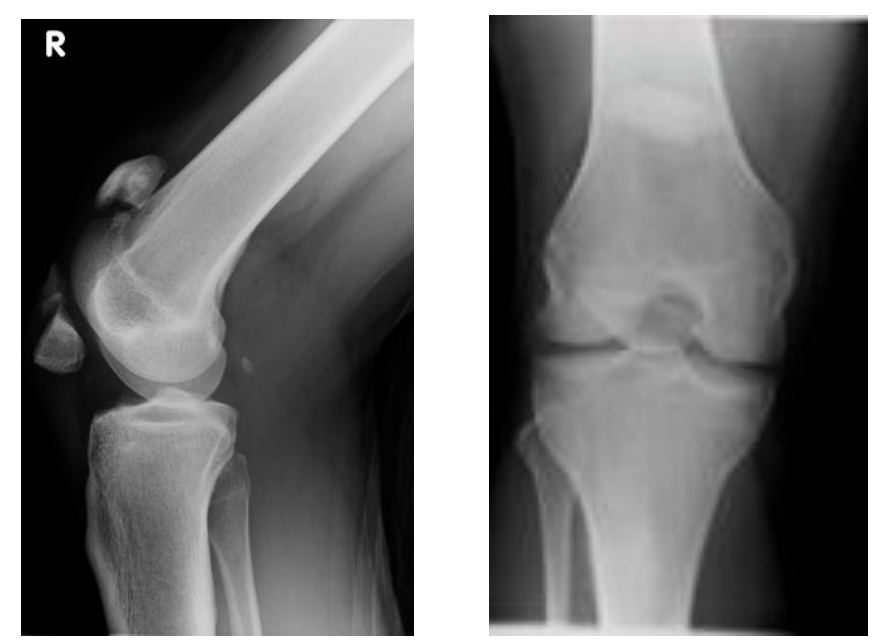

Abbildung 1: Trümmerfraktur der Patella

Die Frakturen betreffen dabei vorwiegend Personen zwischen dem 30. und 60. Lebensjahr (Brill und Hopf 1987), wobei ein sehr geringer Teil von einer beidseitigen Fraktur der Patella betroffen ist (Boström 1972).

Die Patella ist als Teil des Femoropatellargelenks in die Biomechanik des Kniegelenkes integriert. Durch ihre Lage im Streckapparat erhöht sie als größtes Sesambein des menschlichen Skeletts in Form eines Hypomochlions den Hebelarm des Musculus quadriceps femoris und insbesondere beim extendierten Kniegelenk das Drehmoment (Menschik 1974, Maquet 1977, Endler 1980, Hepp 1983) und kompensiert „die bei der Umlenkung der Quadricepssehne entstehenden Druck- und Scherkräfte“" (Debrunner 2005, S. 1042).

Es wurde bereits in verschiedenen Studien (Kaufer 1971, Lennox et al. 1994) festgestellt, dass aus einer Patellektomie eine bis zu 30 - 40\%ige Funktionseinbuße des Musculus quadriceps femoris resultieren kann. Eine vollständige Kompensation der Kraft ist ohne eine operative Wiederherstellung der Patella deshalb nicht wiederherzustellen. 


\subsection{Anatomie des Kniegelenks}

Das Kniegelenk (Articulatio genus) besteht anatomisch aus drei knöchernen Anteilen (Femur, Tibia, Patella), die über Gelenke miteinander verbunden sind. Insgesamt besitzt das Kniegelenk vier Freiheitsgrade, nämlich Flexion/Extension, Ab-/Adduktion, Außen/Innenrotaion und axiale Rotation. Aufgrund von vier anatomischen Drehachsen wird das Knie als Viergelenk bezeichnet (Nägerl et al. 1993, Frosch et al. 2009).

Einerseits ist dies die Articulatio femoropatellaris, welche zwischen den artikulierenden Anteilen des Oberschenkelknochens (Femur) und der Kniescheibe (Patella) besteht. Andererseits setzt sich die Articulatio femorotibialis aus den zwei konvex geformten Femurkondylen und dem Schienbeinkopf (Caput tibiae) zusammen. Insgesamt wird das Kniegelenk durch ligamentäre und muskuläre Anteile stabilisiert.

Durch zwei in das Kniegelenk integrierte Menisken werden im Kniegelenk stattfindende Bewegungen weiterhin unterstützt und geführt (Debrunner 2005). Circa 70 Prozent des tibialen Plateaus werden von den Menisken eingenommen (Fukubayashi und Kurosawa 1980). Diese sowohl bindegewebigen als auch knorpeligen „Puffer“ sind untereinander verbunden und für eine physiologische Gelenkfunktion notwendig.

Die konkaven Menisken kompensieren die im lateralen Tibiaplateau vorzufindende Inkongruenz der Gelenkpartner (Wirth et al. 2005) und verbinden die beidseitig konvexen Femurkondylen mit dem Tibiaplateau, welches medialseitig konkav und lateralseitig konvex geformt ist (Frosch et al. 2009). Außerdem führen die Menisken zu einer vergrößerten Gelenkfläche zwischen dem Femur und der Tibia, wodurch der Spitzendruck im Gelenk gemindert wird. Falls ein Meniskus partiell oder vollständig entfernt worden ist, resultiert dies in einem vergrößerten Druck im Gelenk (Seedhom und Hargreaves 1979).

Bei der schon Anfang des 20. Jahrhunderts beschriebenen Hauptbewegung im Kniegelenk, der Flexions-/Extensionsbewegung, ist zu beachten, dass die beginnende Flexion aus maximaler Extension einen Rollmechanismus darstellt (Zuppinger 1904, Fischer 1907). Ab einer Beugung aus der Standphase von ca. $15-20^{\circ}$ geht dieses Rollen zunehmend in einen Gleitmechanismus über und ab ca. $25^{\circ}$ findet nahezu ausschließlich ein reines Gleiten statt (Wismans et al. 1980, Thomsen et al. 2002, Frosch et al. 2009) 


\subsection{Anatomie und Funktion der Patella}

Die Patella entsteht im Laufe des dritten Lebensjahres aus verschiedenen Ossifikationszentren und verknöchert vollständig in der Adoleszenz. Die endgültige individuelle Form entwickelt sich durch die kontinuierliche Interaktion mit dem femoralen Gleitlager und den vorherrschenden Kräftverhältnissen im Femoropatellargelenk (Schlenzka et al. 1979).

Sie stellt einen bedeutsamen Teil des Femoropatellargelenks dar und ist in den Streckapparat des Beins eingegliedert.

Der apikale Teil wird von der breiten Basis patellae gebildet, nach distal wird die Patella in Richtung des Apex patellae schmaler.

Auf der Vorderseite ist die Patella knöchern und besitzt eine aufgeraute Oberfläche, an der Faseranteile der Quadrizepssehne ansetzen. Im medialen Drittel der Patella befinden sich ca. zehn bis zwölf kleine Öffnungen (Foramina nutricia) longitudinal verlaufender Kanäle für eintretende Blutgefäße, welche hauptsächlich die mittleren und oberen Anteile der Patella versorgen.

Der Apex patellae zeigt an der ventralen und dorsalen Seite Gefäßöffnungen zur Versorgung des distalen Anteils.

Die konkav verlaufende Rückseite ist bis auf die Patellaspitze von einer ca. 5 - $7 \mathrm{~mm}$ breiten hyalinen Knorpelschicht überzogen, welche durch einen medianen Hauptfirst in eine mediale und laterale Gelenkfläche unterteilt wird (Wiberg 1941, Hehne et al. 1981).

Anhand von Defilée-Aufnahmen der Patella können nach Wiberg (1941) aufgrund des Firstes folgende drei Untergruppen von Kniescheibentypen unterschieden werden:

- Typ I: medialer und lateraler Anteil sind gleich groß;

- Typ II: der laterale Anteil ist größer (am häufigsten vorkommender Typ);

- Typ III: der laterale Anteil ist ausgedehnt und konvex, wohingegen der mediale Anteil konkav und deutlich kleiner ist. 
Der hyaline Knorpel ist für die funktionelle Beanspruchung des Kniegelenks von immenser Bedeutung, da nur so der Anpressdruck gegenüber dem femoralen Gleitlager abgefangen werden kann.

Die Patella wird dabei durch muskuläre, sehnige und ligamentäre Stabilisatoren in ihrer Position gehalten, welche für die ordnungsgemäße Funktion des Patellofemoralgelenks von entscheidender Bedeutung sind.

Hier kann man zwischen aktiven (muskulären) und passiven (knöchernen und ligamentären) Stabilisatoren des Kniegelenks unterscheiden (Pförringer und Gorschewsky 2005, Wirth et al. 2005).

Den aktiven Part stellt der Musculus quadriceps femoris mit seinen vier Anteilen dar:

- $\quad$ M. vastus medialis

- M. vastus intermedius

- M. vastus lateralis

- M. rectus femoris (mit Caput longum et breve).

Dabei inseriert der Quadrizeps-Muskel sowohl an der proximal gelegenen Basis der Patella, bildet außerdem das medial und lateral ansetzende Retinaculum patellae und im Bereich des distal gelegenen Apex patellae (Patellaspitze) gehen Faseranteile in die Patellarsehne, welche an der Tuberostias tibiae ansetzt, über.

$\mathrm{Zu}$ den passiven knöchernen und ligamentären Anteilen stellten zuerst Conlan et al. (1993) und später weitere Autoren (Burks et al. 1998, Desio et al. 1998, Hautamaa et al. 1998, Nomura et al. 2000, Smirk und Morris 2003, Steensen et al. 2004) fest, dass bis zu 53 Prozent der medialen Stabilisation durch das Zusammenwirken des medialen femoropatellaren Ligamentums und des $M$. vastus medialis obliquus erreicht werden. Auf der medialen Seite sind drei weitere Bänder zu nennen, die einerseits an der Stabilisation nach medial mitwirken als auch die Luxation nach lateral verhindern sollen:

- das Ligamentum patellofemorale mediale

- das Ligamentum patellotibiale mediale und

- das Retinaculum mediale

Im Gegensatz zu den medialen Anteilen ist auf der lateralen Seite besonders das Retinaculum patellae laterale zu erwähnen, welches den Tractus iliotibialis mit dem lateralen Patellarand verbindet. 
Im Kniegelenk können Flexions-/Extensionsbewegungen von ungefähr 140 - 150 stattfinden (Roaas und Andersson 1982, Wirth et al. 2005), wobei stets verschiedene Anteile der Patella mit der femoralen Gleitfläche artikulieren. Bei kompletter Streckung liegt die Patella im Recessus suprapatellaris und nur ca. 10\% der Patellarückfläche artikulieren mit der Facies patellaris des Femur. Bei Beugung des Kniegelenks ist die Kontaktfläche über eine Distanz von ca. fünf bis sieben Zentimeter (Hehne 1990) in der Rinne zwischen den Femurkondylen (Trochlea femoris) verschiebbar.

Hierbei unterscheiden sich jeweils die artikulierenden Anteile der Patella und des Femurs je nach Neigungswinkel im Kniegelenk und der aufgebrachten Zugkraft des QuadrizepsMuskels. Bei Beugung verschieben sich die Kontaktflächen auf der Patella von distal kontinuierlich in Richtung der apikal gelegenen Basis patellae.

So stehen z. B. bei einem Beugungswinkel von $20^{\circ}$ die distalen, von $90^{\circ}$ die proximalen Anteile der Patella mit der Trochlea femoris und $a b 120^{\circ}$ vor allem die medial und lateral gelegenen Anteile der Patellarückfläche mit den Femurkondylen in Berührung (Goodfellow et al. 1976a, Hehne 1990).

Die laterale Facette der Patella wird bei Flexion mehr belastet als die mediale Facette, die erst bei einer Beugung von ca. $20^{\circ}$ im Kniegelenk mit dem femoralen Gleitlager artikuliert. Bei noch größerer Flexion (bis zur maximalen Beugung) separieren sich die artikulierenden Anteile der Patella in zwei seitliche Teile, die mit den beiden Femurkondylen in Berührung stehen, wohingegen der mediane Anteil der Patella und die Facies articularis patellae sowie die Area inercondylaris femoris voneinander getrennt sind (Hehne 1990, Powers 1998).

Es kommen bestimmte Anteile der medialen Gelenkfläche (Odd-Facette) erst ab einer Flexion von ca. $135^{\circ}$ mit dem medialen Femurkondylus in Kontakt (Goodfellow et al. 1976b). Bei diesem Beugungswinkel gleitet die Patella in der Area intercondylaris und wird durch eine ab ca. $135^{\circ}$ stattfindende axiale Rotation der Patella nach lateral verdreht, wodurch sich die Odd-Facette an den medialen Kondylus anlegt.

Dazu gegensätzlich verlagern sich die Kontaktflächen des femoralen Gleitlagers von proximal (Trochlea femoris) nach distal (Femurkondylen) (Feller at al. 2007). Hierbei wird die Position der Kniescheibe durch die seitlich gelegenen Retinaculae, Quadrizeps-Anteile und das Lig. patellae determiniert.

Bei größerer Beugung übernimmt sukzessiv die Trochlea femoris die Führung der Kniescheibe und die ligamentären Strukturen geraten in den Hintergrund (Feller et al. 2007). 

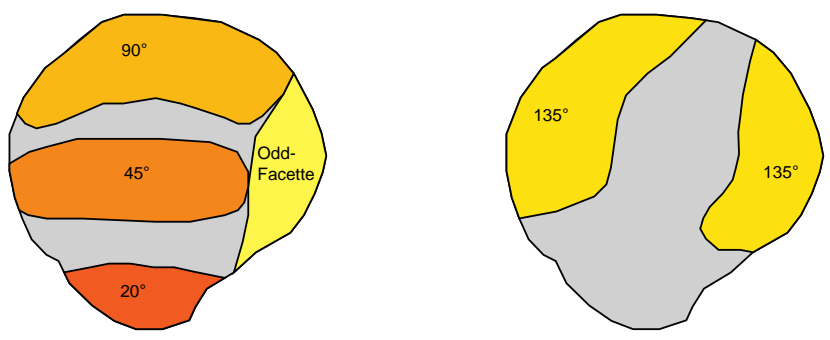

Abbildung 2: Kontaktflächen der Patella mit dem Femur Vgl.: Goodfellow et al. 1976a, S. 288

Mit freundlicher Genehmigung der British Editorial Society of Bone and Joint Surgery

In der beschriebenen Kinematik des Kniegelenks kann der auf die Patellarückfläche ausgeübte Druck (Anpressdruck in der femoralen Trochlea $=$ Facies patellaris femoris) bei festgestelltem Unterschenkel und Flexion im Kniegelenk bis zu 300kp und mehr betragen (Kapandji 2006). In Extensionsstellung des Kniegelenkes überwiegen die Distraktionskräfte des Streckapparates auf das Kniegelenk, wohingegen bei Beugung im Kniegelenk die Dreipunktbiegekräfte in den Vordergrund treten (Rüter et al. 2008).

\subsection{Klassifikation der Patellafrakturen}

Frakturen der Patella treten vor allem im Rahmen von sog. „Dashboard-Injurys“ auf, welche eine direkte Krafteinwirkung auf das gebeugte Kniegelenk bedeuten. Diese treten besonders bei frontalen Autounfällen oder auch bei Stürzen auf das gebeugte Kniegelenk auf.
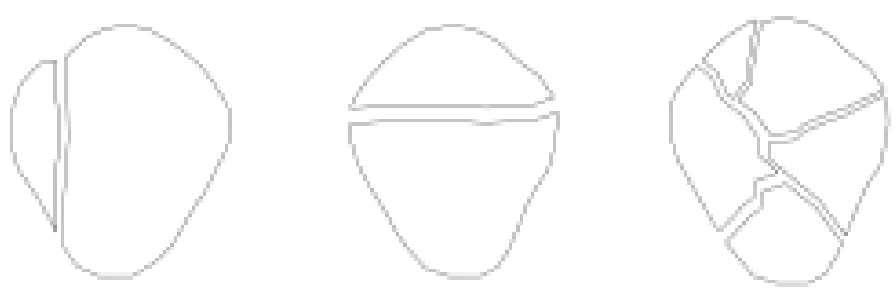

Abb. 3: Längs-, Quer- und Trümmerfraktur der Patella 
Von geringer Häufigkeit sind Frakturen der Patella bei spontaner Beugung des Kniegelenks bei kontrahiertem Musculus quadriceps femoris (3-Punkt-Beugebelastung) oder Frakturen, die nach teilweiser Entfernung der Patellarsehne zur Materialgewinnung als Ersatzplastik für das vordere Kreuzband auftreten (Christensen und Jakob 1992). Bei Patellarückflächenersatz im Rahmen einer Totalendoprothesenimplantation am Kniegelenk stellt die Patellafraktur ebenfalls eine seltene Kompilkation dar (Arnold et al. 1998).

Vereinfacht werden folgende Bruchformen der Patella unterschieden:

- Querfrakturen

- Längsfrakturen

- Sternfrakturen

- Trümmerfrakturen

- $\quad$ Abrissfrakturen (Pol- und Randfrakturen)

- Osteochondrale Frakturen (Flakes).

Querfrakturen nehmen dabei im Durchschnitt einen Anteil von etwa $70-80 \%$ aller Patellafrakturen ein (Boström 1972, Galla und Lobenhoffer 2005), jedoch wird in neuerer Literatur beschrieben, dass aufgrund der sozioökonomischen Entwicklung eine steigende Anzahlen an Mehrfragmentären-/ Trümmerfrakturen zu beobachten ist (Vicenzi et al. 1985, Benger et al. 1986).

Klinisch imponiert eine Patellafraktur meist mit einem Erguss im Kniegelenk, einem Hämarthros und einer deutlich schmerzbedingten Bewegungseinschränkung.

Eine Patellafraktur kann allerdings bei kleinen Fragmenten (Weinberg und Tscherne 2006) oder auch im Rahmen eines Polytraumas (Hou et al. 2002) leicht übersehen werden.

Die Frakturen können nach dem Klassifikationssystem von Speck und Regazzoni (1994) klassifiziert werden, die den Verlauf und die Form der Fraktur beschreiben:

- Typ A: Längsfrakturen

- A1: nicht dislozierte Längsfraktur

- A2: dislozierte Längsfraktur

- A3: Längsfraktur mit Zusatzfragment 
- Typ B: Querfrakturen

- B1: Polabriss ohne Gelenkbeteiligung

- B2: einfache Querfraktur

- B3: Querfraktur mit Zusatzfragment oder doppelte Querfraktur

- Typ C: Mehrfragmentfrakturen

- C1: Mehrfragmentfraktur ohne Dislokation

- C2: Mehrfragmentfraktur (Dislokation kleiner 2mm)

- C3: Mehrfragmentfraktur (Dislokation größer 2mm).

nach: Stürmer Leitlinien Unfallchirurgie, 1999

Außerdem kann man Patellafrakturen nach dem System der AO-Klassifikation (Arbeitsgemeinschaft für Osteosynthesefragen) einteilen (Müller et al. 1979). Dieses Klassifikationssystem legt eine größere Bedeutung auf eine eventuelle Gelenkbeteiligung der Patellafraktur.

Dabei werden drei Gruppen gebildet, die jeweils Untergruppen besitzen:

Die Gruppe A besteht aus gelenkfreien Frakturen (zwei Untergruppen), Gruppe B bezeichnet Frakturen mit partieller Frakturierung des Gelenks (fünf Untergruppen) und die Gruppe C definiert Frakturen, bei denen es zur vollständigen Destruktion der Gelenkfläche kommt (Neun Untergruppen). Außerdem wird der Patellafraktur zur Codierung die Nummer 34 zugeordnet. So entspricht z.B. 34-C3.3 einer vollständig intraartikulär liegenden Trümmerfraktur.

Eine operative Versorgung einer Patellafraktur ist generell indiziert bei folgenden Faktoren / Gegebenheiten:

- Frakturen mit einer Dislokation der Fragmente von 2mm oder mehr

- Stufenbildung in der Gelenkfläche (Facies articularis patellae)

- unverschobene Frakturen bei fehlender Streckfähigkeit oder mangelnder funktioneller Behandelbarkeit

- offene Verletzungen 
- im Rahmen der operativen Versorgung anderer, kniegelenksnaher Verletzungen sinnvoll.

(Böhler 1961, Böstman et al. 1981, Johnson 1991, Gosal et al. 2001)

\subsection{Problemstellung}

Eine Patellafraktur kann theoretisch mittels einer konservativen oder operativen Therapie behandelt werden. Aufgrund der imminent wichtigen Beteiligung der Patella am Femoropatellargelenk ist eine konservative Therapie nur zu rechtfertigen, wenn unverschobene oder nur minimal dislozierte Fragmente bei ausreichender Stabilität im Kniegelenk vorliegen (Wirth et al. 2005), jedoch sind hierbei höhere Re-Frakturraten als mögliche Komplikation zu beachten (Rees und Thompson 1985). Außerdem kann bei einer isolierten Polfraktur ohne Gelenkbeteiligung ein konservatives Verfahren gewählt werden (Sperner und Wanitschek 1989, Dietz et al. 2009).

Ansonsten ist die offene Reposition und interne Fixation (ORIF) als Verfahren der Wahl für die Versorgung einer Patellafraktur anzusehen.

Ziel jeder Operation sollte eine möglichst anatomisch exakte Wiederherstellung der retropatellaren Gleitfläche sein, um somit postoperativ physiologische Gleitvorgänge des Femoropatellargelenkes zu gewährleisten (Dick et al. 1975, Lennox 1994) und um das Risiko einer eventuellen Femoropatellararthrose zu minimieren (Labitzke und Rehn 1973, Rogge et al. 1985).

Nur bei einer sehr großen Destruktion der Patella ist eine Teil- oder vollständige Patellektomie als eine Möglichkeit der Versorgung in Erwägung zu ziehen. Dabei werden nach Entfernung der Patella die Sehne des Quadrizeps-Muskels und das Ligamentum patellae vernäht, wodurch zumindest eine gewisse Restfunktion im Kniegelenk erhalten werden kann.

Es ist stets eine möglichst zeitnahe Versorgung (innerhalb der ersten sechs Stunden nach dem Unfallereignis) anzustreben oder bei einer offenen Fraktur eine notfallmäßige Operation durchzuführen (Wendl et al. 2002).

Bei einer operativen Versorgung einer Patellafraktur kann zwischen drei Osteosyntheseverfahren unterschieden werden: einer Schraubenosteosynthese, einer Zuggurtungsosteosynthese oder einer Kombination aus vorhandenen Osteosynthesematerialien wie K-Drähten, Cerclagen, Schrauben etc.. 
Die erste Möglichkeit besteht in der Versorgung mittels einer Schraubenosteosynthese. Bei diesem Verfahren werden die Fragmente nach manueller Reposition mittels Zugschrauben fixiert.

Hierdurch werden die Fragmente ausreichend komprimiert und eine hohe Belastungsstabilität erreicht (Wendl et. al 2002).

Bei einer Zuggurtung, die vor allem bei Querfrakturen zum Einsatz kommt, wird durch die Operation eine ausreichende primäre Stabilität erreicht und der auf den Fragmenten lastende Zug in Druckkräfte umgewandelt (Wendl et al. 2002). Meist werden zwei vertikal verlaufende Kirschnerdrähte von proximal nach distal parallel zueinander eingebracht und eine vertikal ausgerichtete Cerclage aus einem Edelstahldraht in Form einer Acht hinzugefügt, wodurch eine interfragmentäre Kompression resultiert.

Deshalb kann an eine Zuggurtungsosteosynthese eine frühzeitige physiotherapeutische Behandlung angeschlossen werden (Rüter et al. 2008).

Die dritte Möglichkeit besteht in einer Materialkombination, die besonders bei komplizierten Frakturen wie einer Trümmerfraktur zum Einsatz kommt.

Hier ist das Vorgehen des Operateurs von der individuellen Situation des Patienten abhängig. Der behandelnde Arzt versucht mit einer Auswahl von Kirschnerdrähten, Schauben, Zuggurtungsdrähten oder z.B. einer Cerclage eine möglichst optimale Wiederherstellung der Anatomie anzustreben.

Das Ziel jeder der genannten Operationen sollte eine möglichst anatomisch exakte Wiederherstellung der retropatellaren Gleitfläche sein, um somit postoperativ physiologische Gleitvorgänge des Femoropatellargelenkes zu gewährleisten (Dick et al. 1975, Lennox et al. 1994).

Nach einer operativen Versorgung eines Bruches bestehen Risiken für eventuelle postoperative Komplikationen. Wie bei allen Operationen können insbesondere Materialdislokationen, Heilungsstörungen, Knorpelschädigungen und Pseudarthrosen als Komplikationen nach einer operativ versorgten Patellafraktur auftreten.

Als eine weitere mögliche Komplikation bei einer Patellafraktur kann es zu einem hyperdensen Bereich in den ossären Anteilen der Kniescheibe kommen, die einem osteonekrotischen Umbau entsprechen.

Dies wurde bei der nachfolgenden operativen Versorgung (Scapinelli 1967), nach dem Einsetzen einer Kniegelenksendoprothese (Cameron und Huffer 1985, Holtby und Grosso 1996) oder nach einer Verabreichung von Corticosteroiden (Elkayam et al. 1996) beobachtet. 
So wurde beispielsweise die Entstehung von Osteonekrosen nach einer Patellaquerfraktur in etwa $25 \%$ (41 von 162 Patienten) der mittels einer Zuggurtungsosteosynthese behandelten Verletzungen beschrieben (Scapinelli 1967).

Diese minderversorgten Areale treten bei einer Fraktur im mittleren Drittel der Patella fast immer im oberen (proximalen) Fraktursegment auf, da dieses von der Blutzufuhr abgeschnitten ist, wohingegen das untere Segment noch ausreichenden arteriellen Zufluss erhält.

Diese Folgeschäden eines Bruches der Kniescheibe resultieren aus der zugrunde liegenden Blutversorgung der Patella, welche über ein extra- und ein intraossäres Gefäßsystem sichergestellt wird.

Die extraossär liegenden Arterien bestehen aus einem vernetzten Blutgefäßsystem, welches ringförmig um die Patella angelegt ist. Aus mehreren arteriellen Zuflüssen bildet sich ein konvergent auf die Mitte der Patella zulaufendes Gefäßnetz (Rete patellae).

Das extraossäre Blutgefäßsystem wird unter anderem durch einen absteigenden Zustrom aus der Arteria femoralis (A. descendens genicularis) und einen aufsteigendem Zufluss aus der Arteria tibialis anterior (A. recurrens tibialis anterior) gespeist.

Der Hauptzustrom des Gefäßnetzes erfolgt über mehrere Zuflüsse aus der A. poplitea (A. superior medialis genus, $A$. superior lateralis genus, $A$. inferior medialis genus und $A$. inferior lateralis genus).

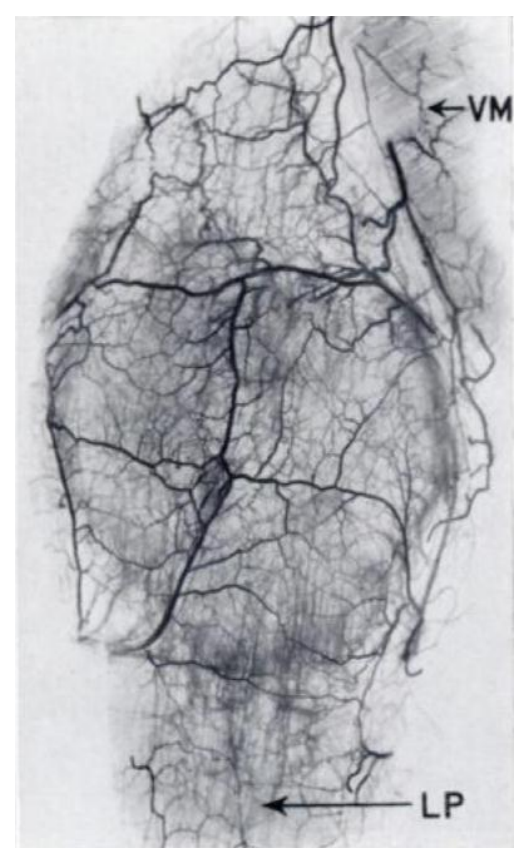

Abbildung 4: Gefäßstruktur der Patella aus: Scapinelli 1967, S. 563 Mit freundlicher Genehmigung der British Editorial Society of Bone and Joint Surgery 
Die zwei superioren Äste der A. poplitea verlaufen horizontal vor der Quadrizepssehne am oberen Patellapol und anastomosieren mit Anteilen der A. descendens genicularis. Am unteren Patellapol treffen die beiden inferioren Anteile der A. poplitea hinter dem Ligamentum patellae zusammen und geben Äste zur Versorgung des unteren Drittels der Patella ab.

Gemeinsam verbinden sich die Anteile der superioren und inferioren Zuflüsse zu einem vor der Patella liegenden Gefäßnetz, aus dem die Anteile des inneren Gefäßssystems gespeist werden.

Das intraossär gelegene Blutgefäßsystem besteht aus zwei verschiedenen Zuflüssen:

Einerseits treten im Bereich des mittleren Drittes der Patella die zuführenden Blutgefäße über auf der Vorderseite liegende Foramina nutricia in die Patella ein und verlaufen intraossär schräg nach apikal bis zur Knochen-Knorpel-Grenze. Die hier eintretenden Blutgefäße verzweigen sich innerhalb der Patella und versorgen die oberen zwei Drittel der Patella (Scapinelli 1967).

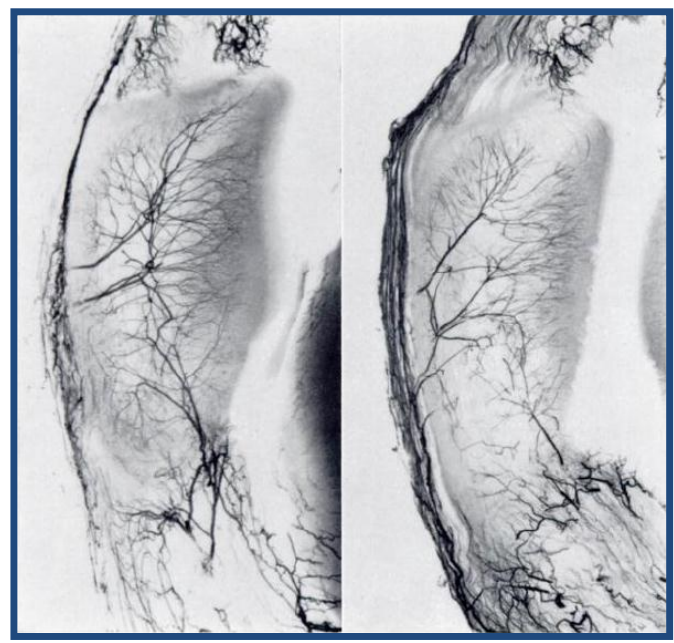

Abbildung 5: Versorgung der Patella aus: Scapinelli 1967, S. 565

Mit freundlicher Genehmigung der British Editorial Society of Bone and Joint Surgery

Andererseits gibt es außerdem einen intraossären Zustrom aus dem Bereich des distalen Pols: Hinter dem Ligamentum patellae verbinden sich die Aa. geniculares inferiores medialis und lateralis und treten zwischen dem Ligamentum patellae und der Gelenkfläche in die Patella ein. Über diesen Zustrom wird primär der distale Pol versorgt.

Über Anastomosen innerhalb der Patella wird eine Verbindung zwischen den beiden intraossär verlaufenden Gefäßsystemen gebildet.

Über die Ränder der Patella werden keine weiteren Zuflüsse hergestellt, da diese nur als Ansatzpunkte für Bänder und Sehnen dienen. 
Bei einer Quer- oder Trümmerfraktur kann es aufgrund der Blutversorgung der Patella zu Heilungsstörungen kommen, denn bei einer Querfraktur wird die Patella in einen proximalen und distalen Anteil separiert.

Der proximale Anteil wird durch den Bruch von jeglicher Blutversorgung abgeschnitten, wohingegen die Versorgung des distalen Pols auch weiterhin über einen der beiden Zuströme sichergestellt ist (Scapinelli 1967, Theodorou et al. 2001).

Diese nun minderperfundierten Areale können zu einem nekrotischen Umbau des Knochens führen, der sich radiologisch als röntgendicht bzw. hyperdens darstellt.

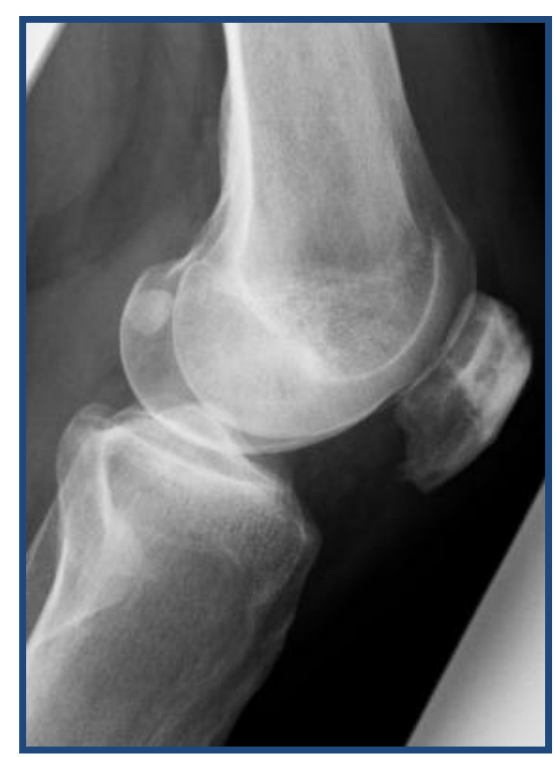

Abbildung 6: Hyperdenses Areal des proximalen Pols

Erste Anzeichen für entstehende hyperdense Areale können in der Regel radiologisch zwischen dem ersten und zweiten Monat nach dem Trauma bemerkt werden, wobei das Maximum der radiologisch sichtbaren Verdichtung nach zwei bis drei Monaten erreicht wird (Bohr und Larsen 1965).

Neben den aufgrund der Fraktur entstehenden minderperfundierten Arealen, konnte gezeigt werden, dass auch durch die bei einer Querfraktur standardmäßig verwendeten Kirschnerdrähte nekrotische Areale resultieren können (Franssen et al. 2008) .

Denn aufgrund der mittels eines Bohrers eingebrachten Drähte kann sich die Temperatur im Bereich um den Kirschnerdraht auf über $50^{\circ}$ Celsius erhöhen, wodurch es zu der Entstehung von Hitzenekrosen kommen kann. Demgegenüber sei die nicht übliche Einbringung des 
Kirschnerdrahtes mittels eines Hammers der Variante mit einem Bohrer überlegen, da keine nekrotischen Areale resultierten und die Kirschnerdrähte außerdem fester im Knochen fixiert seien.

Des Weiteren ist nicht gesichert, inwieweit eine retropatellare Stufenbildung nach der Operation das klinische Ergebnis und die Zufriedenheit der Patienten beeinflusst.

So führt beispielsweise schon eine ein Millimeter kleine Stufe im retropatellaren Gleitlager zu einer erhöhten Reibung zwischen Patella und korrespondierenden, femoralen Gelenkanteilen, so dass eine nicht exakt anatomisch rekonstruierte Gelenkfläche durch eine ständige Mikrotraumatisierung in einer posttraumatischen Arthrosebildung resultieren kann (Reynaud et al. 1996, Freuler und Brunner 1975).

Denn bei einer größer als zwei Millimeter verbliebenen Gelenkstufe sollte eine Revisionsoperation in Erwägung gezogen werden, um einer potentiell frühzeitig entstehenden Arthrose im Femoropatellargelenk entgegenzuwirken (Dietz et al. 2009). Hier sei darauf verwiesen, dass die Arthrose die häufigste Gelenkerkrankung der Welt darstellt und ungefähr mehr als die Hälfte aller chronischen Krankheiten bei Patienten über 60 Jahren darstellt (Petersson 1996). Davon werden ca. 80\% als primäre (idiopathische) Arthrosen definiert, deren Ursache nicht zu benennen ist, und nur ca. $20 \%$ können sekundären Arthrosen (z.B. posttraumatisch) zugeordnet werden (Buckwalter et al. 2004).

Dabei verwendeten wir die von Kellgren und Lawrence (1957) entwickelten Kriterien zur Beurteilung einer Arthrose, die vier Stufen der chronisch-progredienten arthrotischen Entwicklung beinhalten.

Um ein möglichst zufriedenstellendes Ergebnis zu erzielen, sollte eine standardmäßige Nachbehandlung angeschlossen werden, welche idealerweise aus einer frühfunktionellen Nachbehandlung mit Teilbelastung besteht.

Nach stattgefundener Wundheilung und bei unkompliziertem postoperativen Verlauf kann nach 6 Wochen das Bewegungsausmaß kontinuierlich bis zur vollständigen Aufhebung gesteigert werden. Die allgemeinen Heilungsfortschritte sollen mittels radiologischer Aufnahmen in regelmäßigen Abständen bis zur definitiven knöchernen Heilung kontrolliert werden. 


\subsection{Zielsetzung der Studie}

Ziel der durchgeführten retrospektiven klinischen Studie ist die Evaluation der klinischen und radiologischen Ergebnisse von Patienten mit Patellafrakturen, die im Zeitraum von 1998 2008 in der Klinik für Unfallchirurgie, Plastische und Wiederherstellungschirurgie der Universitätsmedizin Göttingen operativ behandelt wurden.

Dabei sollen die verschiedenen Operationsverfahren verglichen und eventuelle Beschwerden der Patienten dokumentiert werden.

Außerdem sollen die postoperativ angefertigten radiologischen Aufnahmen auf hyperdense Areale untersucht werden sowie daraufhin, ob diese Auswirkungen auf das klinische Ergebnis der Patienten haben.

Insbesondere die Ausbildung hyperdenser Areale nach Patellafraktur fand bisher in der Literatur kaum Beachtung. In Medline gelistete Publikationen finden sich dazu bisher nicht.

Bereits vorliegende Röntgenaufnahmen aus Routinekontrolluntersuchungen der Patienten werden auf mögliche postoperative Auffälligkeiten der Patella untersucht (Stufen in der retropatellaren Gelenkfläche, hyperdense Areale, verbliebene knöcherne Defekte, Arthrosezeichen) und es wird überprüft, ob Zusammenhänge zwischen den radiologischen Gegebenheiten und der Patientenzufriedenheit sowie international anerkannten klinischen Scores (siehe unten) bestehen. Im Rahmen der Studie werden keine neuen Röntgenbilder angefertigt. 


\section{Material und Methoden}

\subsection{Patientenkollektiv}

Es wurde eine retrospektive Untersuchung aller Patienten durchgeführt, die zwischen 1998 und 2008 in der Abteilung Unfall-, Plastische und Wiederherstellungschirurgie der Universitätsmedizin Göttingen aufgrund einer Patellafraktur operativ versorgt wurden.

Im oben genannten Zeitraum wurden insgesamt 110 Patienten wegen einer Patellafraktur operativ behandelt und nach Genehmigung durch die Ethikkommission (Antragsnummer 13/2/09) wurden alle Patienten schriftlich über die Studie informiert und anschließend telefonisch zu einer einmaligen Nachuntersuchung in die Universitätsmedizin Göttingen eingeladen.

Ausschlusskriterien stellen die konservative Therapie oder die Operation ohne Einbringen von Osteosynthesematerialien dar. Außerdem wurden Patienten mit einer beidseitigen Patellafraktur ausgeschlossen, da eine Verfälschung der Ergebnisse zu erwarten war.

Eine frühere auf der Gegenseite erlittene Fraktur, die zum Zeitpunkt der aktuellen Fraktur vollständig ausgeheilt war, stellte kein Ausschlusskriterium dar.

\subsection{Anamnestische Daten}

Vor der klinischen Nachuntersuchung der Patienten wurden die vorliegenden Akten und radiologischen Aufnahmen der Patienten ausgewertet.

Dabei wurden folgende Daten aus den Krankenakten notiert:

- Geschlecht

- Alter beim Unfall

- Unfallursache

- Untersuchungsbefund bei der Primärversorgung (Erguss, Schmerzen, Schwellung, etc.)

- Zeit zwischen Unfall und operativer Versorgung in Stunden

- Art der osteosynthetischen Versorgung

- Nachbehandlungsschema. 
Die Unfallursachen wurden dabei in Verkehrsunfälle, Stürze, Anpralltraumata, Absturztraumata und in Knieverdrehtraumata unterteilt.

Aus den radiologischen Aufnahmen wurde die Fraktur nach dem Schema von Speck und Regazzoni (1994) klassifiziert und die operative Versorgung begutachtet.

Dabei wurden die bereits durchgeführten Aufnahmen auf eventuelle arthrotische Anzeichen im Kniegelenk, postoperativ verbliebene Stufen sowie Defekte oder hyperdense Areale in der Patella untersucht.

Um eventuelle Folgen von hyperdensen Arealen in der Patella auf das klinische Ergebnis der Patienten nachzuweisen, wurden die Nachuntersuchungsbögen der Studienteilnehmer in Gruppen aufgeteilt. Dabei wurde zwischen dem Vorliegen eines hyperdensen Areals und keiner pathologischen Auffälligkeit unterschieden. Bei der klinischen Nachuntersuchung wurden die weiter unten aufgeführten Scores abgefragt.

\subsection{Operationsmethoden}

Die operative Versorgung wurde stets von einem unfallchirurgischen Facharzt und einem inn unterstützenden weiteren Facharzt oder Assistenten durchgeführt.

Standardmäßig wurde der Patient in einer Vollnarkose behandelt, auf dem Rücken gelagert, eine Blutsperre am Oberschenkel des operierten Beins mit einem maximalen Druck von bis zu $350 \mathrm{mmHg}$ angelegt und das operierte Areal großflächig nach etwaiger Hautrasur chirurgisch desinfiziert.

Der Zugangsweg zur Eröffnung des Kniegelenks wurde jeweils den Gegebenheiten des Patienten angepasst. Dabei wurden sowohl die Fraktur des Patienten als auch die Weichteilsituation berücksichtigt, wie z.B. bei einer offenen Fraktur oder ausgeprägten Kontusionszonen.

Als Möglichkeiten stehen dem Operateur der mediale parapatellare, der laterale parapatellare Längsschnitt und der mediane Querschnitt zur Auswahl. Als Operationsmethoden, die standardmäßig über eine offene Reposition und interne Stabilisierung durchgeführt wird (ORIF = Open Reduction and Internal Fixation), stehen unterschiedliche Osteosyntheseverfahren zur Verfügung.

Alle Osteosyntheseverfahren beginnen nach Eröffnung des Kniegelenkes mit einer Spülung des Kniebinnenraumes und einer Freilegung und Inspizierung der Frakturflächen.

Es wird prinzipiell zwischen drei Osteosyntheseverfahren unterschieden: einer Schraubenosteosynthese, einer Zuggurtungsosteosynthese oder einer Kombination aus vorhandenen Osteosynthesematerialien wie K-Drähten, Cerclagen, Schrauben etc.. 
Die Schraubenosteosynthese, die vor allem bei Längsfrakturen aber auch bei Querfrakturen zum Einsatz kommt, beginnt mit einer manuellen Reposition, wonach die Fragmente durch eine spitze Repositionszange fixiert werden.

Anschließend werden zwei parallel verlaufende, horizontale Zugschrauben eingebracht (bei einer Querfraktur vertikal verlaufend). Dabei ist darauf zu achten, dass der Verlauf der Schrauben die hyaline Patellarückflache ausspart und nur im knöchernen Anteil der Patella verläuft. Außerdem müssen der Kopf der Schraube und das Gewinde in unterschiedlichen Fragmenten positioniert werden, da nur so eine angemessene Kompression der Fragmente und ein positives Operationsergebnis erzielt werden kann (Durst und Arnold 1994, Debrunner 2005).
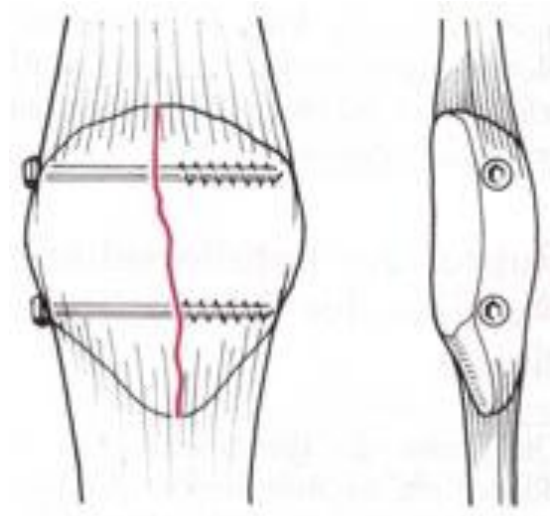

Abbildung 7: Schraubenosteosynthese bei einer Längsfraktur mit Aussparung der hyalinen Rückfläche. Vgl.: Durst 1997, S. 127 Mit freundlicher Genehmigung des Schattauer Verlags Stuttgart

Die Enden der Schrauben sollten sich möglichst noch in der Kortikalis der Patella befinden, da so eine Weichteilirritation verhindert werden kann (Galla und Lobenhoffer 2005).

Als zweites Verfahren hat sich die Zuggurtungsosteosynthese etabliert, die vielfach modifiziert werden kann. Hier beginnt der Operateur ebenfalls mit einer möglichst exakten manuellen Reposition der Frakturfragmente. Anschließend werden die zwei parallel und längsgerichteten Kirschner-Drähte (1,6-2,0 mm Dicke) eingebracht um die eine Zuggurtungsschlinge (1,0-1,2 mm Dicke) gelegt wird, welche das Ligamentum patellae und die Sehne des Musculus quadriceps femoris penetriert. Die Zuggurtung kommt auf der Vorderfläche der Patella zu liegen, wird hinter den Enden der umgebogenen Kirschnerdrähte fixiert und gespannt, wodurch die Fraktur und die Zuggurtung in Position gehalten werden. 


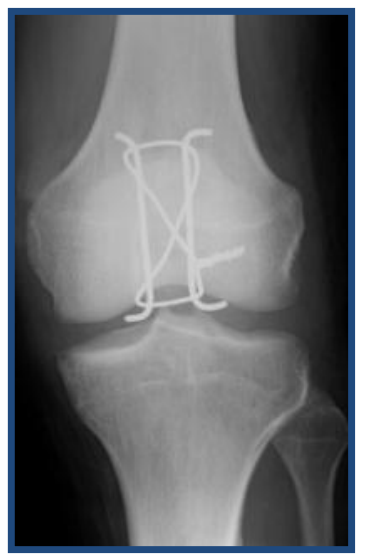

Abbildung 8: Studienteilnehmer mit einer Zuggurtungsosteosynthese

Durch ein geeignetes Osteosyntheseverfahren wie die Zuggurtung wird eine ausreichende Primärstabilität erreicht und der auf den Fragmenten lastende Zug in Druckkräfte umgewandelt werden (Wendl et al. 2002).

Deshalb kann an eine Zuggurtungsosteosynthese eine frühzeitige physiotherapeutische Behandlung angeschlossen werden (Rüter, Trentz, Wagner 2008).

Als Variation können z.B. statt Kirschner-Drähten Schrauben benutzt werden, um die eine Drahtcerclage in Form einer Acht gelegt wird oder man verwendet vertikal verlaufende kanülierte Schrauben, durch welche die Drahtcerclage verläuft.

Die dritte Möglichkeit besteht in einer Materialkombination, die besonders bei komplizierten Frakturen wie einer Trümmerfraktur zum Einsatz kommen.

Hier ist das Vorgehen des Operateurs von der individuellen Situation des Patienten abhängig. Der behandelnde Arzt versucht mit einer Auswahl von Kirschnerdrähten, Schrauben, Zuggurtungsdrähten oder z.B. einer Cerclage eine möglichst optimale Wiederherstellung der Frakturelemente anzustreben. Falls es zu einer komplizierten Trümmerfraktur gekommen ist und die einzelnen Fragmente nicht mehr stabil mittels einer Osteosynthese fixiert werden können, müssen diese Fragmente oder im Zweifelsfall die gesamte Patella entfernt werden.

Nach Beendigung der Frakturversorgung wird in das eröffnete Kniegelenk eine RedonDrainage eingelegt, um eventuelle Nachblutungen zu drainieren. Wenn nötig wird die Gelenkkapsel vernäht, eine Subkutannaht vorgenommen, die Hautinzision durch eine Hautnaht verschlossen und der Operationsbereich durch einen sterilen Verband abgedeckt. 
Das Ziel jeder der genannten Operationen sollte eine möglichst anatomisch exakte Wiederherstellung der retropatellaren Gleitfläche sein, um postoperativ physiologische Gleitvorgänge des Femoropatellargelenkes zu gewährleisten (Dick et al.1975, Lennox et al. 1994) und postoperative Komplikationen zu minimieren.

Denn bei einer größer als zwei Millimeter verbliebenen Gelenkstufe sollte eine Revisionsoperation in Erwägung gezogen werden, um einer potentiell frühzeitig entstehenden Arthrose im Femoropatellargelenk entgegenzuwirken (Dietz et al. 2009).

\subsection{Nachbehandlung}

Die Nachbehandlung besteht aus einer multidisziplinären Nachsorge des Patienten, um ein optimales postoperatives Ergebnis zu erreichen und enthält folgende Aspekte:

$\begin{array}{ll}\text { - } & \text { Frühfunktionelle Bewegung } \\ \text { - } & \text { Physiotherapie } \\ \text { - } & \text { Schmerzbehandlung } \\ \text { - } & \text { SPM (passive Bewegungsschiene) } \\ \text { - } & \text { Stützverband bei wenig kooperativen Patienten } \\ \text { - } & \text { Thromboseprophylaxe individuell fortführen. }\end{array}$

aus: Stürmer Leitlinien Unfallchirurgie 1999

Die in der Abteilung für Unfallchirurgie, Plastische- und Wiederherstellungschirurgie durchgeführte Nachbehandlung besteht aus einer 6-wöchigen postoperativen Sohlenkontaktbelastung, die durch die Anlage einer ROM-Schiene (Range of motion) mit $0 / 0 / 60^{\circ}$ für vier Wochen und anschließend mit 0/0/90 für zwei Wochen unterstützt wird. Der Patient versucht unter Unterstützung einer physiotherapeutischen Abteilung vorsichtig die Kontaktbelastung zu erhöhen. In der Regel kann, unter Berücksichtigung des Patienten, des Frakturtyps und der osteosynthetischen Versorgung, eine Vollbelastung sechs Wochen nach der Operation erreicht werden. Eine kontinuierliche Steigerung des Bewegungssaumaßes sollte durchgeführt werden. Es sollte nach sechs Wochen mindestens ein Bewegungsausmaß von $90^{\circ}$ Flexion erreicht werden, da nur so längerfristige Bewegungseinschränkungen verhindert werden können (Galla und Lobenhoffer 2010). Eine Entfernung des Osteosynthesematerials ist nicht zwingend erforderlich, sondern nur nach Übereinstimmung mit der Leitlinie Implantatentfernung der Deutschen Gesellschaft für Unfallchirurgie e.V. (DGU) zu empfehlen. 


\subsection{Postoperative Komplikationen}

Die in der folgenden Liste aufgeführten Probleme im postoperativen Ablauf der operativen Versorgungen wurde als eine Komplikation gewertet.

Neben den allgemeinen Problemen wie Wundheilungsstörungen, Infektionen oder Sensibilitätsstörungen stehen bei einer Patellafraktur folgende Komplikationen im Vordergrund:

- Unfallbedingter traumatischer Knorpelschaden (Chondropathia patellae)

- $\quad$ Lockerung und Wanderung des Osteosynthesematerials

- Materialbruch

- Metallallergie (Chrom-Nickel-Stahl)

- $\quad$ Frakturdislokation und Osteonekrosen

- Gelenkinfektion

- $\quad$ Bursitis präpatellaris (Schleimbeutelentzündung)

- Bewegungseinschränkung des Kniegelnks

- Irritation durch Osteosynthesematerialien

- $\quad$ Retropatellare Stufenbildung im Gelenk, Defekte

- $\quad$ CPRS (Morbus Sudeck)

- $\quad$ Patella baja (Tiefstand der Patella)

- $\quad$ Fixierte Patella

aus: Stürmer Leitlinien Unfallchirurgie 1999

Außerdem stellen nach einer nicht adäquaten operativen Versorgung der Patellafraktur Pseudarthrosen eine mögliche Komplikation dar (Freuler at el. 1975). 


\subsection{Klinische Nachuntersuchung}

Alle Patienten, die sich bereit erklärt hatten an der Studie teilzunehmen, wurden gebeten den aus folgenden Anteilen bestehenden Nachuntersuchungsbogen im Rahmen einer klinischen Untersuchung auszufüllen.

\subsubsection{VASS (Visuell numerische Analogskala Schmerz)}

Hierbei sollen die Patienten einen vertikalen Strich über eine horizontale Linie ziehen, die ihre Schmerzen in den letzten Tagen vor der Nachuntersuchung beschreibt. Diese Linie entspricht zehn Zentimetern und reicht von „0“ (keine Schmerzen) bis „10“ (der maximal vorstellbare Schmerzen) Punkten.

\subsection{Kniescores}

Zur Feststellung der postoperativen Situation des Patienten und seines Kniegelenks wurden international verwendete Scores benutzt. Es wurden der AKSS (Insall et al. 1989), der Tegner-Aktivitätsscore (Tegner und Lysholm 1985), der WOMAC-Score (Bellamy und Buchanan 1982) und der Lysholm-Score (Lysholm und Gillquist 1982) verwendet.

\subsubsection{AKSS (American Knee Society Score)}

Der 1989 von der American Knee Society vorgestellte Score (Insall et al. 1989) hat sich international als ein Standard-Score zur Verwendung bei Patienten mit Knieendoprothesen und Beschwerden im Bereich des Kniegelenkes etabliert.

Dieser Score ist in zwei Teile unterteilt:

Der erste Teil erfasst etwaige Schmerzen des Patienten sowie das Bewegungsausmaß im Kniegelenk (Stabilität, Beugekontraktur, Streckdefizit), wohingegen der zweite Teil einen Funktionsscore darstellt, der darüber Auskunft gibt inwieweit der Patient gehen oder Treppen steigen kann und ob er dabei Hilfsmittel benutzen muss.

In beiden Abschnitten können jeweils 100 Punkte erreicht werden, was dem maximalen Ergebnis von 200 entspricht, welches eine völlig beschwerdefreie Symptomatik des 
Patienten bedeutet. Der minimal erreichbare Score für den Patienten beträgt -70 Punkte, da z.B. negative Punktzahlen für ein Streckdefizit und Beugekontrakturen vergeben werden.

\subsubsection{Tegner-Aktivitätsscore}

Bei diesem Score werden über eine numerische Einschätzung maximal zehn Punkte für die sportliche Aktivität und berufliche Situation des Patienten vergeben.

Dabei wird jeweils ein Wert vor und nach der Operation ermittelt, um so eine Differenz zwischen diesen beiden Werten zu ermitteln und vergleichen zu können inwieweit sich Veränderungen in der sportlichen bzw. alltäglichen Situation des Patienten aufgrund der Fraktur und anschließenden Operation ergeben haben.

Das Aktivitätsniveau des Patienten ist in elf Unterpunkte unterteilt. Das Maximum von zehn Punkten erfüllt der Patient wenn er Fußball auf nationalem und/oder internationalem Niveau ausübt. Der Durchschnitt von fünf Punkten ist z.B. definiert als die berufliche Tätigkeit eines Bauarbeiters und das andere Ende der Skala von null Punkten entspricht der Bettlägerigkeit oder Rente wegen Knieproblemen.

Der postoperative Wert des Patienten wurde immer während der Nachuntersuchung erhoben, wohingegen der präoperative Status des Patienten retrospektiv durch den Patienten selbst eingeschätzt wurde.

\subsubsection{WOMAC-Score (Western Ontario \& McMaster Universities Osteoarthritis Index)}

Der WOMAC-Score dient der Registrierung von Problemen der Patienten in alltäglichen Situationen und stellt darüber hinaus physische Funktionseinschränkungen fest (Stucki et al. 1996).

Er wird von Bellamy und Buchanan (1986) als ein valider Score beschrieben, der bei Patienten mit Hüft- und Gonarthrose zuverlässig die klinische Situation des Patienten widerspiegelt.

Der WOMAC-Score umfasst drei Anteile:

Der erste Bereich umfasst fünf Fragen nach Schmerzen, der zweite Teil besteht aus zwei Fragen nach einer eventuellen Steifigkeit im Kniegelenk und der dritte Abschnitt enthält 17 Fragen zu Schwierigkeiten in alltäglichen Situationen des Patienten.

Hierbei waren die Patienten jeweils dazu angehalten in der jeweiligen numerischen Graduierungsskala ein Kreuz in eines der elf Kästchen zu setzen von null (z.B. keine 
Schmerzen) bis zehn (z. B. maximal vorstellbarer Schmerz). Somit ergeben sich als maximal erreichbare Punktzahl in Teil A 50, in Teil B 20 und in Teil C 170 Punkte, wodurch sich eine maximale Gesamtpunktzahl von 240 ergibt. Das beste Ergebnis und somit völlige Beschwerdefreiheit entsprechen null Punkte, die maximale Punktzahl von 240 dem negativsten Ergebnis.

Zur statistischen Auswertung wurde weiterhin ein WOMAC-Score-Gesamtindex gebildet. Dabei wurden die einzelnen Bereiche durch die Anzahl der jeweils entsprechenden Fragen dividiert, wodurch sich für jede der drei Skalen ein Wertebereich von null bis maximal zehn Punkten ergab. Anschließend wurden die drei Ergebnisse addiert und durch drei dividiert, woraufhin sich ein WOMAC-Score zwischen null und zehn Punkten ergab. Somit kann allen drei Teilen des WOMAC-Scores eine gleiche Bedeutung beigemessen werden sollte.

Wir verwendeten den für den deutschen Sprachraum von Stucki et al. 1996 evaluierten Fragebogen, der sich aufgrund seiner klaren Strukturierung als sehr verständlich für den Patienten erwiesen hat.

\subsubsection{Lysholm-Score}

Der Lysholm-Score dient der subjektiven Evaluation von Funktionalität und Stabilität des Kniegelenkes im klinischen Alltag.

Bei diesem Score kann man zwischen einem funktionellen und einem symptom-orientierten Anteil unterscheiden, die zusammen einer Maximalpunktzahl von 100 entsprechen.

Bewertung der erreichten Punktzahl:

\begin{tabular}{|c|c|}
\hline $100-91$ Punkte & Sehr gut \\
\hline $77-90$ Punkte & Gut \\
\hline $68-76$ Punkte & Befriedigend \\
\hline$<68$ Punkte & Unbefriedigend \\
\hline
\end{tabular}

Tabelle 1: Bewertung des erreichten Ergebnisses im Lysholm-Score

Zu dem funktionellen Teil, der ein Viertel der Gesamtpunktzahl ausmacht, zählen Fragen ob der Patient hinkt, er das Knie voll belasten kann, wie er Treppen steigt und wie er in die Hocke gehen kann. Der symptom-orientierte Anteil, der drei Viertel der Gesamtpunktzahl ausmacht, summiert sich aus Fragen zu einer Blockierung im Kniegelenk, ob eine Instabilität vorliegt, ob das Knie anschwillt oder ob Schmerzen bei dem Patienten bestehen. 
Die verschiedenen Parameter des Lysholm-Scores werden folgendermaßen gewichtet:

\begin{tabular}{|c|c|c|}
\hline Bereich: & Parameter: & Maximale Punktzahl: \\
\hline & & 5 \\
\hline Funktionell & Hinken & 5 \\
\hline & Belastung & 10 \\
\hline & Treppensteigen & 5 \\
\hline & Hocken & 15 \\
\hline Symptomatisch & Blockierung & 25 \\
\hline & Instabilität & 25 \\
\hline & Schmerzen & 10 \\
\hline & Schwellung & Max. 100 Punkte \\
\hline & &
\end{tabular}

Tabelle 2: Verteilung des funktionellen und symptomatischen Teils

\subsection{Zufriedenheit der Patienten}

Abschließend wurde nach der subjektiven Zufriedenheit der Patienten gefragt. Dabei sollten die Patienten ihr Operationsergebnis bewerten von null (absolut unzufrieden) bis zehn Punkten (vollkommen zufrieden). Diese Skala entspricht einer visuellen Analogskala und die Patienten sollten einen vertikalen Strich über einen horizontale Linie ziehen, welcher später einem Wert zugeordnet wurde.

\subsection{Radiologische Auswertung}

Zur Beurteilung der unabhängig von der Studie vorher durchgeführten Röntgenaufnahmen wurde ein eigener Röntgen-Score entwickelt. Dieser umfasst die Feststellung von Stufen, Defekten, Nekrosen und Arthrosezeichen.

Auf das Problem einer Stufe in der Gelenkfläche wurde bereits früher eingegangen. Wegen der großen Bedeutung der Wiederherstellung der Kongruenz wurde der Parameter Stufe in vier Untergruppen unterteilt.

Das beste Ergebnis stellt keine Stufe im postoperativen Röntgenbild dar, welches mit drei Punkten gewertet wurde. Zwei Punkte gab es für eine Stufe von bis zu einem Millimeter. Bei 
einer Stufe von mehr als einem bis zu drei Millimetern wurde ein Punkt vergeben und abschließend wurde für eine Stufe von mehr als drei Millimetern kein Punkt mehr vergeben.

Als nächstes wurde ein eventuelles osteonekrotisches Areal begutachtet. Das ideale Ergebnis entsprach keinen Anzeichen für eine Nekrose, was mit der maximalen Punktzahl von vier Punkten gewertet wurde.

Wenn die nekrotischen Areale bis zu einen Zentimeter der Patella ausfüllten, wurden drei Punkte vergeben. Entsprach die Nekrose mehr als einem bis zu drei Zentimetern wurden dafür zwei Punkte vergeben. Wenn die Patella zu fünfzig Prozent nekrotische Areale besaß wurde nur ein Punkt vergeben. Bei einer völlig nekrotischen Patella als schlechtestes Ergebnis wurde kein Punkt mehr angerechnet.

Der dritte Punkt stellte die Beurteilung eines postoperativ verbliebenen Defektes in der Patella dar. War idealerweise kein Defekt zu erkennen, konnte dafür ein Punkt erlangt werden. Falls jedoch ein Defekt im postoperativen Röntgenbild persisierte wurde dafür kein Punkt vergeben.

Abschließend wurden die radiologischen Bilder der Patienten auf arthrotische Anzeichen untersucht. Anhand der Einteilung von Kellgren und Lawrence wurden die Studienteilnehmer aufgrund ihrer radiologischen Bilder in vier Gruppen eingeteilt.

\begin{tabular}{|l|l|l|}
\hline Arthrosezeichen & Stadium 1 & $\begin{array}{l}\text { Geringe subchondrale Sklerosierung } \\
\text { Keine Osteophyten } \\
\text { Keine Gelenkspaltverschmälerung }\end{array}$ \\
\cline { 3 - 4 } & Stadium 2 & $\begin{array}{l}\text { Geringe Gelenkspaltverschmälerung. } \\
\text { Beginnende Osteophytenbildung. } \\
\text { Angedeutete Unregelmäßigkeit der Gelenkfläche }\end{array}$ \\
\cline { 2 - 3 } & Stadium 3 & $\begin{array}{l}\text { Ausgeprägte Osteophytenbildung } \\
\text { Gelenkspaltverschmälerung } \\
\text { Deutliche Unregelmäßigkeit der Gelenkfläche }\end{array}$ \\
\cline { 2 - 3 } & Stadium 4 & $\begin{array}{l}\text { Ausgeprägte Gelenkspaltverschmälerung bis zur } \\
\text { vollständigen Destruktion } \\
\text { Deformierung/Nekrose der Gelenkpartner }\end{array}$ \\
\hline
\end{tabular}

Tabelle 3: Stadien der arthrotischen Entwicklung nach: Kellgren \& Lawrence, 1957, S. 494 


\subsection{Histologische Untersuchung der Patella}

Nachdem bei einem Patienten radiologisch ein hyperdenses Areal in der Patella entdeckt wurde, sind anschließend bei diesem Patienten im Rahmen einer weiteren operativen Versorgung Proben aus der Patella entnommen worden. Es wurden dabei Proben aus dem proximalen (hyperdensen Bereich) und aus dem distalen Anteil der Patella gewonnen und nach Fixierung anschließend mit einer Hämatoxylin-Eosin-Färbung gefärbt.

\section{Ergebnisse}

\subsection{Patientenkollektiv}

Im Zeitraum von 1998 bis 2008 wurden in der Abteilung Unfallchirurgie, Plastische und Wiederherstellungschirurgie der Universitätsmedizin Göttingen 110 Patienten aufgrund von Frakturen der Patella behandelt.

Von diesen 110 Patienten erklärten sich 62 Patienten (56\%) dazu bereit an der Studie teilzunehmen und wurden deshalb in einem einmaligen Follow-Up untersucht und gebeten einen Nachuntersuchungsbogen auszufüllen. Zwei Patienten wurden im Nachhinein von der Studie ausgeschlossen. Ein Patient hatte sich zeitgleich beide Kniescheiben gebrochen und eine Patientin wurde zwar operiert, jedoch wurde die Patellafraktur nicht über eine Osteosynthese versorgt, da die Fraktur nicht die Knorpel-Knochen-Grenze tangierte. Deshalb bestand das Patientenkollektiv abschließend aus 60 Patienten (55\%).

Von den 48 (44\%) Patienten, die nicht an der Studie teilgenommen haben, wurden sieben Patienten nicht kontaktiert, da fünf der Patienten nicht operativ versorgt wurden und zwei Patienten sich während eines Unfalls beide Kniescheiben gebrochen haben.

Somit wurde noch bei 41 (37\%) Personen versucht, sie zu kontaktieren, wovon jedoch 21 Patienten weder per Post noch telefonisch erreicht werden konnten. Weitere sechs Patienten erklärten, dass sie nicht bereit waren an der Studie teilzunehmen und verweigerten ihre Zustimmung.

Fünf Patienten waren zwischen der Operation und der Kontaktaufnahme bereits verstorben. Zwei weitere Patienten waren aufgrund einer Erkrankung an Morbus Alzheimer nicht in der Lage an der Studie teilzunehmen. 
Zwei weitere Patienten waren mittlerweile im Ausland wohnhaft (Dänemark und Japan), weshalb eine Teilnahme an der Studie nicht möglich war. Aufgrund von beruflichen Gründen und daraus resultierenden terminlichen Schwierigkeiten konnten weitere zwei Patienten nicht an der Studie partizipieren.

Ein Patient wollte an der Studie teilnehmen, doch aufgrund einer Herz-Kreislauf-Erkrankung war es inm nicht möglich die Anreise zur Untersuchung auf sich zu nehmen. Ein anderer Patient musste sich aufgrund einer peripheren arteriellen Verschlusskrankheit ( $p A V K)$ sein operiertes Bein amputieren lassen, weshalb die Evaluation nicht möglich war. Eine weitere Patientin befand sich wegen einer psychiatrischen Erkrankung in einer geschlossenen Anstalt zur Rehabilitation wodurch eine Untersuchung ihrer postoperativen Ergebnisse folglich ebenfalls nicht möglich war.

Somit konnten die Ergebnisse von 60 Patienten untersucht werden, die über eine der drei operativen Methoden versorgt wurden.

Die Patienten wurden in drei Gruppen entsprechend ihrem Frakturtypus unterteilt:

Es fanden sich unter den 60 Patienten sieben mit einer Längsfraktur (Frakturtyp A), 28 mit einer Querfraktur (Frakturtyp B) und 25 mit einer Trümmerfraktur (Frakturtyp C), wobei sich unter den 60 Patienten insgesamt 43 Männer und 17 Frauen befanden .

Unter den Patienten mit einer Längsfraktur befanden sich sechs Männer und eine Frau. In der Gruppe B nahmen elf Frauen und 17 Männer an der Studie teil und in der dritten Gruppe (Frakturtyp C) nahmen fünf Frauen und 20 Männer teil.

\begin{tabular}{|c|c|c|c|c|c|}
\hline Fraktur A & 7 & Fraktur B & 28 & Fraktur C & 25 \\
\hline A1 & 3 & B1 & 4 & C1 & 1 \\
\hline A2 & 2 & B2 & 18 & C2 & 7 \\
\hline A3 & 2 & B3 & 6 & C3 & 17 \\
\hline
\end{tabular}

Tabelle 4: Übersicht über die Frakturformern der Studienteilnehmer

Im Mittel betrug das Alter der Patienten 45,48 (+/- 18,51) Jahre. Zwischen den einzelnen Gruppen konnte in Bezug auf den Altersdurchschnitt kein signifikanter Unterschied festgestellt werden. 
Das durchschnittliche Alter der Patienten, die eine Längsfraktur erlitten, betrug 53,86 (+/17,25) Jahre. Die Patienten mit einer Querfraktur wiesen ein durchschnittliches Alter von 46,64 (+/- 18,1) Jahren auf und die Personen mit einer Trümmerfraktur hatten ein durchschnittliches Alter von 41,84 (+/- 19,07) Jahren.

In Bezug auf den Body-Mass-Index (BMI), der bei allen 60 Patienten erhoben wurde, konnte kein signifikanter Unterschied zwischen den drei Gruppen festgestellt werden.

In der Gruppe A lag der durchschnittliche BMI bei $25,45(+/-3,88) \mathrm{kg} / \mathrm{m}^{2}$, in Gruppe B bei $25,48(+/-4,84) \mathrm{kg} / \mathrm{m}^{2}$ und in der dritten Gruppe bei $23,63(+/-3,19) \mathrm{kg} / \mathrm{m}^{2}$.

Die Zeitspanne zwischen der Fraktur und der operativen Versorgung der Patienten wurde ebenfalls erhoben. Dabei konnte ein signifikanter Unterschied zwischen der Frakturgruppe $C$ gegenüber den Frakturgruppen $A(p=0,011)$ und $B(p=0,021)$ festgestellt werden.

In der Gruppe A betrug die durchschnittliche Zeit zwischen der Fraktur und der Operation im Durchschnitt 144 (+/- 134,8) Stunden, in der Gruppe B 121,8 (+/- 135,6) Stunden und in der Gruppe C 51,78 (+/- 63,5) Stunden.

Abschließend wurde das Patientenkollektiv noch auf die Zeit zwischen der Fraktur und der klinischen Nachuntersuchung (Follow-Up) hin untersucht.

Insgesamt fand die Nachuntersuchung im Mittel 60,6 (+/- 33,88) Monate nach der Operation des Patienten/ der Patientin statt.

Die erste Gruppe (Frakturtyp A) wurde im Mittel 66,71 (+/- 34,77) Monate nach der Operation untersucht.

Die zweite Gruppe wurde durchschnittlich 71,19 (+/- 33,05) Monate später untersucht und die Gruppe des Frakturtyps C im Durchschnitt 51,78 (+/- 35,2) Monate danach. 


\begin{tabular}{|c|c|c|c|c|c|}
\hline & $\begin{array}{c}\text { Frakturtyp } \\
\text { A }\end{array}$ & $\begin{array}{c}\text { Frakturtyp } \\
\text { B }\end{array}$ & $\begin{array}{c}\text { Frakturtyp } \\
\text { C }\end{array}$ & $\begin{array}{c}\text { Keine } \\
\text { Nekrose }\end{array}$ & Nekrosen \\
\hline Anzahl & 7 & 28 & 25 & 54 & 6 \\
\hline Weiblich & 1 & 11 & 5 & 16 & 1 \\
\hline Männlich & 6 & 17 & 20 & 38 & 5 \\
\hline $\begin{array}{l}\text { Alter } \\
\text { (Jahre) }\end{array}$ & $\begin{array}{c}53,86 \\
(+/-17,25 ; 22- \\
76)\end{array}$ & $\begin{array}{c}46,64 \\
(+/-18,1 ; 14- \\
76)\end{array}$ & $\begin{array}{c}41,84 \\
(+/-19,07 ; 11- \\
84)\end{array}$ & $\begin{array}{c}45,83 \\
(+/-18,73 ; 11- \\
84)\end{array}$ & $\begin{array}{c}42,33 \\
(+/-17.7 ; 19- \\
64)\end{array}$ \\
\hline $\begin{array}{l}\text { BMI } \\
\left(\mathrm{kg} / \mathrm{m}^{2}\right)\end{array}$ & $\begin{array}{c}25,45(+/- \\
3,88 ; 19,47- \\
31,74)\end{array}$ & $\begin{array}{c}25,48(+/- \\
4,84 ; 16,97- \\
40,09)\end{array}$ & $\begin{array}{c}23,63(+/- \\
3,19 ; 17,01- \\
29,68)\end{array}$ & $\begin{array}{c}24,64(+/- \\
4,32 ; 16,97- \\
40,09)\end{array}$ & $\begin{array}{c}25,27(+/- \\
2,30 ; 22,27- \\
28,35)\end{array}$ \\
\hline $\begin{array}{l}\text { OP- } \\
\text { Zeitpunkt }\end{array}$ & $\begin{array}{c}144,7(+/- \\
134,8 ; 46- \\
412,5)\end{array}$ & $\begin{array}{c}124(+/- \\
135,6 ; 1-525)\end{array}$ & $\begin{array}{c}51,8(+/- \\
63,5 ; 3-235)\end{array}$ & $\begin{array}{c}92,1(+/- \\
104,4 ; 1-504)\end{array}$ & $\begin{array}{c}124(+/- \\
201 ; 3-525)\end{array}$ \\
\hline $\begin{array}{l}\text { Follow- } \\
\text { Up } \\
\text { (Monate) }\end{array}$ & $\begin{array}{c}66,71(+/- \\
34,77 ; 30-115)\end{array}$ & $\begin{array}{c}71,19(+/- \\
33,05 ; 23- \\
123)\end{array}$ & $\begin{array}{c}61,44(+/- \\
35,2 ; 19-136)\end{array}$ & $\begin{array}{c}63,45(+/- \\
33,16 ; 19- \\
136)\end{array}$ & $\begin{array}{c}95(+/- \\
28,71 ; 46- \\
133)\end{array}$ \\
\hline
\end{tabular}

Tabelle 5: Übersicht über die Zusammensetzung der 3 Fraktur-Gruppen und der 2 Patientenkollektive (Keine Nekrose, Nekrose)

(Für Alter, BMI, OP-Zeitpunkt und Follow-Up wurden jeweils Mittelwert, Standardabweichung und der jeweilige minimale und maximale Wert notiert.)

Die 60 an der Studie teilnehmenden Patienten wurden osteosynthetisch mit einem der nachfolgend angeführten Verfahren versorgt:

\begin{tabular}{|l|c|c|c|}
\hline & Frakturtyp A & Frakturtyp B & Frakturtyp C \\
\hline Zuggurtung & 1 & 20 & 13 \\
\hline Schraubenosteosynthese & 6 & 3 & 4 \\
\hline Kombination & - & 3 & 6 \\
\hline Andere Versorgungen & - & 1 Cerclage, 1 K-Draht & 2x McLoughlin \\
\hline Insgesamt 60 & 7 & 28 & 25 \\
\hline
\end{tabular}




\subsection{Verletzungsursachen}

Es fanden sich unter den 60 analysierten Patienten insgesamt 21 (35\%) die sich durch Verkehrsunfälle ihre Frakturen zuzogen. Zehn Patienten waren in einen Verkehrsunfall mit ihrem Auto involviert, sieben Patienten stürzten mit ihrem Motorrad und vier Patienten sind mit einem Fahrrad in einen Unfall verwickelt worden.

Insgesamt 30 (50\%) Patienten sind bei einfachen frontalen Stürzen auf ihre Knie gefallen und haben sich dabei ihre Kniescheibe frakturiert (Sturz auf das flektierte Knie mit direktem Anprall der exponiert liegenden Patella).

Fünf (8\%) Patienten zogen sich ihre Verletzung durch ein direktes Anpralltrauma zu (stumpfe Gewalt). Bei vier der Patienten passierte dieses während eines Fußballspiels durch einen Tritt des Gegners gegen die Kniescheibe. Bei dem fünften Patienten kam es im Rahmen einer tätlichen Auseinandersetzung zu mehrfachen Schlägen mit einer Eisenstange gegen seine Kniescheibe, wodurch folglich eine Trümmerfraktur bei dem Patienten resultierte.

Drei $(5 \%)$ der Patienten waren in ein Absturztrauma verwickelt und stürzten z.B. aus einer Höhe von acht Metern auf den Boden, wodurch sie sich ihre Fraktur zuzogen.

Ein Patient zog sich seine Fraktur beim Tischtennisspielen zu. Er berichtete darüber, dass es im Rahmen eines Knieverdrehtraumas zu einer Luxation der Patella kam und diese dabei frakturierte.

\begin{tabular}{|c|c|c|}
\hline Verkehrsunfälle & $\begin{array}{c}21 \\
\text { Autounfall 10 } \\
\text { Motorradunfall 7 } \\
\text { Fahrradunfall 4 }\end{array}$ & $35 \%$ \\
\hline Sturz & 30 & $50 \%$ \\
\hline Anpralltrauma & 5 & $8 \%$ \\
\hline Absturztrauma & 3 & $5 \%$ \\
\hline Knieverdrehtrauma & 1 & $2 \%$ \\
\hline
\end{tabular}

Tabelle 7: Übersicht über die Verletzungsursachen der Studienteilnehmer 
Unter den 60 Studienteilnehmern fanden sich insgesamt jeweils vier Patienten, die im Rahmen eines Polytraumas oder mit einer offenen Fraktur in das Universitätsklinikum eingeliefert wurden.

\subsection{Klinische Nachuntersuchung}

Im Rahmen der klinischen Nachtunersuchung wurden die Patienten in der Universitätsmedizin Göttingen untersucht. Dabei wurde zu Beginn die visuell numerische Analogskala Schmerz abgefragt und anschließend die klinischen Scores erhoben.

\subsubsection{Visuell numerische Analogskala Schmerz (VASS)}

Bei der visuell numerischen Analogskala Schmerz bewerteten in der Gruppe A vier der sieben Patienten (57\%) ihre Beschwerden mit Null, was vollständiger Schmerzfreiheit entspricht. Zwei Patienten (29\%) gaben ihre Schmerzen mit Eins an und ein Patient (14\%) ordnete seine Schmerzen bei Sechs an. Der Mittelwert lag für Gruppe A bei 1,14 (+/- 2,19).

In Gruppe B gaben zwölf der 28 Patienten (43\%) ihre Schmerzen auf der Skala mit null Punkten an. Zwei Patienten (7\%) ordneten sich bei Eins ein, vier Patienten (14\%) bei zwei Punkten, jeweils zwei Patienten (7\%) bei drei und vier Punkten. Vier Patienten (14\%) gaben sich fünf Punkte, ein Patient (4\%) gab sich sechs Punkte und ein Patient ordnete sich bei sieben Punkten ein. Der Mittelwert lag für die Gruppe B bei 2,03 (+/-2,25).

In der Gruppe C konnten bei zehn der 25 Patienten (40\%) null Punkte auf der numerischen Skala notiert werden. Drei Patienten (12\%) gaben sich einen, sechs Patienten (24\%) zwei, vier Patienten (16\%) gaben sich drei und jeweils ein Patient (4\%) gab sich vier und sieben Punkte auf der numerischen Skala. Der Mittelwert lag für die Gruppe C bei 1, $52(+/-1,71)$.

\begin{tabular}{|c|c|c|c|}
\hline Frakturtyp & A & B & C \\
\hline Mittelwert & 1,14 & 2,03 & 1,52 \\
\hline Standardabweichung & 2,19 & 2,25 & 1,71 \\
\hline
\end{tabular}

Tabelle 8: Überblick über die Ergebnisse in der visuell numerischen Analogskala Schmerz 


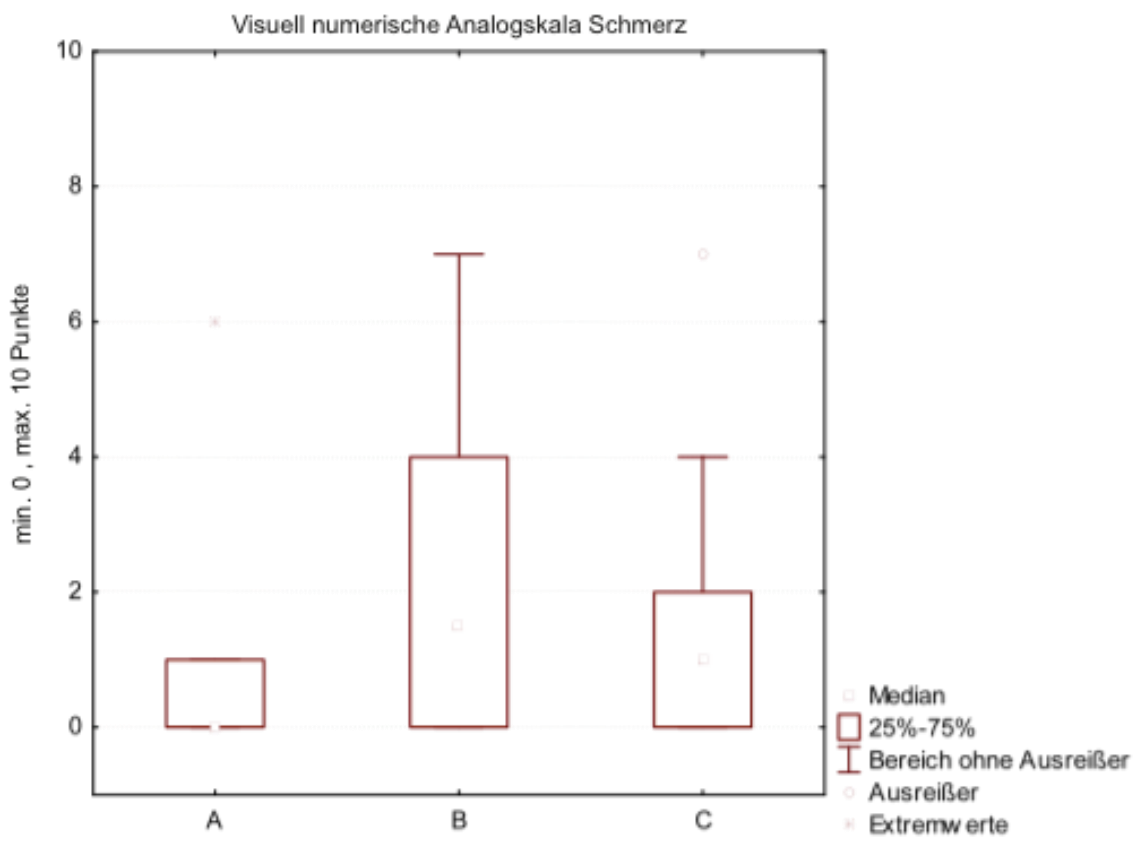

Abbildung 9: Darstellung der Ergebnisse der visuell numerischen Analogskala Schmerz

Zwischen den drei Frakturtypen kann hinsichtlich der subjektiven Einschätzung der Schmerzen zum Zeitpunkt der jeweiligen Nachuntersuchung kein signifikanter Unterschied ( $p$ $=0,530)$ festgestellt werden.

\subsection{Kniescores}

\subsubsection{American Knee Society Score (AKSS)}

In dem American Knee Society Score erreichten die Patienten der Gruppe A Ergebnisse zwischen minimal 149 und maximal 195 Punkten.

Zwei der sieben Patienten erreichten ein exzellentes Ergebnis mit minimal 194 Punkten, vier Patienten erzielten ein gutes Ergebnis mit 170 bis 180 Punkten und ein Patient erreichte ein zufriedenstellendes Ergebnis mit 149 Punkten. Der Mittelwert der Patienten in Gruppe A lag bei 177 Punkten (+/- 15,55).

Die Patienten der Gruppe B erreichten Werte zwischen minimal 82 und der maximalen Anzahl von 200 Punkten. Insgesamt erzielten acht Patienten ein exzellentes Ergebnis, wobei zwei Patienten sogar die Maximalpunktzahl von 200 erreichen konnten. 
Gute Ergebnisse konnten von sieben Patienten erreicht werden. Dabei wurden Werte zwischen minimal 169 und maximal 188 Punkten erzielt.

Acht Patienten erreichten zufriedenstellende Ergebnisse im American Knee Sociey Score. Dabei umfassten die erreichten Punktzahlen Werte zwischen minimal 130 und maximal 160. Fünf Patienten konnten nur ein unbefriedigendes Ergebnis erzielen, wobei minimal 82 und maximal 129 Punkte in diesem Wertebereich erzielt wurden.

Der durchschnittliche Wert der Patienten in Gruppe B lag bei 160 Punkten (+/-35,94).

Die Gruppe C erreicht im American Knee Society Score Werte zwischen 102 und maximal 200 Punkten. Dabei konnten insgesamt sieben exzellente Ergebnisse erzielt werden, worunter drei Mal die Maximalen 200 Punkte erzielt wurden.

In der Gruppe konnten zehn Patienten versammelt werden, die ein gutes Ergebnis erzielten und Werte zwischen 170 und 187 Punkten erreichten.

Ein zufriedenstellendes Ergebnis wurde von sechs Patienten erreicht. Die Werte der Patienten lagen zwischen minimal 143 und maximal 165 Punkten.

Bei zwei Patienten wurde ein unbefriedigendes Ergebnis notiert. Ihre Werte lagen bei 102 und 103 Punkten. Im Mittel erreichten die Patienten der Gruppe C 171,9 Punkte (+/- 25,91).

\begin{tabular}{|c|c|c|c|}
\hline & Gruppe A & Gruppe B & Gruppe C \\
\hline $\begin{array}{c}\text { Exzellent } \\
\mathbf{1 9 0}-\mathbf{2 0 0} \text { Punkte }\end{array}$ & $2(29 \%)$ & $8(29 \%)$ & $7(28 \%)$ \\
\hline $\begin{array}{c}\text { Gut } \\
\mathbf{1 6 8}-\mathbf{1 8 9} \text { Punkte }\end{array}$ & $4(57 \%)$ & $7(22 \%)$ & $10(40 \%)$ \\
\hline $\begin{array}{c}\text { Zufriedenstellend } \\
\mathbf{1 3 0}-\mathbf{1 6 7} \text { Punkte }\end{array}$ & $1(14 \%)$ & $8(29 \%)$ & $6(21 \%)$ \\
\hline $\begin{array}{c}\text { Unbefriedigend } \\
\mathbf{0}-\mathbf{1 2 9} \text { Punkte }\end{array}$ & $0(0 \%)$ & $5(18 \%)$ & $2(8 \%)$ \\
\hline
\end{tabular}

Tabelle 9: Überblick über die Verteilung im American Knee Society Score (AKSS) 


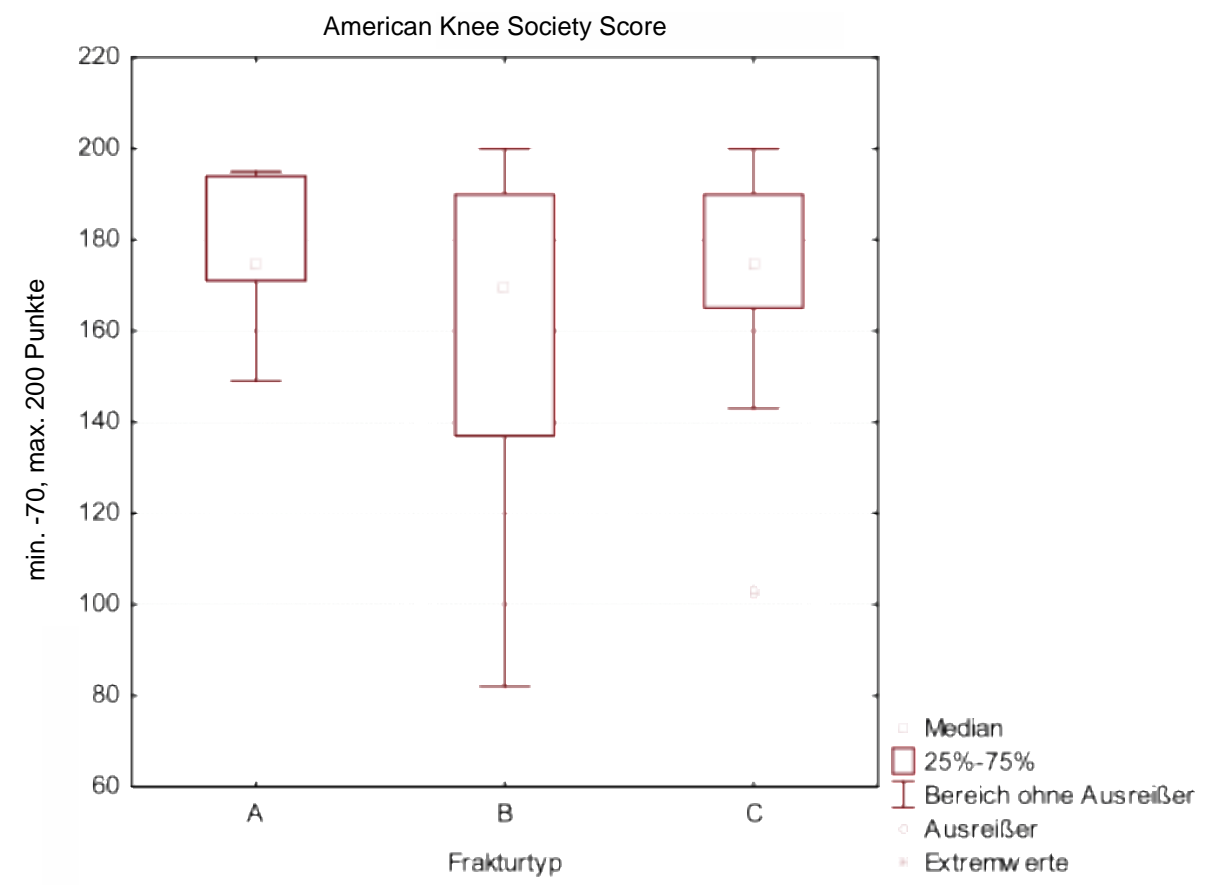

Abbildung 10: Darstellung der Ergebnisse im American Knee Society Score (AKSS)

\begin{tabular}{|c|c|c|c|}
\hline Frakturtyp & A & B & C \\
\hline Mittelwert & 177 & 160 & 171,88 \\
\hline Standardabweichung & 15,55 & 35,94 & 25,91 \\
\hline
\end{tabular}

Tabelle 10: Überblick über die Ergebnisse im American Knee Society Score

Zwischen den drei Frakturtypen kann nach Untersuchung der Ergebnisse des American Knee Society Scores zum Zeitpunkt der jeweiligen Nachuntersuchung kein signifikanter Unterschied $(p=0,446)$ festgestellt werden.

\subsubsection{Tegner-Aktivitätsscore}

Die Patienten der Gruppe A erreichten vor ihrer Patellafraktur minimal drei bis maximal neun Punkte im Tegner-Aktivitätsscore. Im Mittel lag die Gruppe A bei 4,57 Punkten (+/- 1,99) präoperativ.

Nach der Operation gaben die Patienten Werte von minimal drei bis maximal neun Punkten an, wodurch der durchschnittliche Wert bei 4,43 (+/- 2,07) Punkten lag.

Somit erreichten sechs von sieben Patienten (86\%) aus der Gruppe ihren präoperativen Wert und nur ein Patient verschlechterte sich um einen Punkt auf drei. 
In der Gruppe B umfasste der Score präoperative Werte von minimal zwei bis maximal neun Punkte. Dabei lag der durchschnittliche Wert präoperativ bei 4,93 (+/-1,84) Punkten.

Postoperativ konnte bei den Patienten wieder ein Wertebereich von minimal zwei bis maximal neun Punkten erreicht werden. Allerdings konnte auch in dieser Gruppe ein leicht verminderter Wert bei der postoperativen Bestimmung des Tegner-Scores festgestellt werden. Der postoperative Wert lag im Mittel bei 4,07 (+/- 2,12) Punkten und entsprach Werten von minimal null bis maximal neun Punkten.

Insgesamt konnten in der Gruppe B 15 der 28 Patienten ihren präoperativen Wert wieder erreichen, was $54 \%$ entspricht.

In der dritten Gruppe konnte im Mittel präoperativ ein Wert von 5,28 (+/- 1,65) Punkten erzielt werden. Dieser Mittelwert ergab sich aus Werten, die minimal drei und maximal neun Punkten entsprachen.

Nach der Operation konnten die Patienten im Mittel einen Wert von 4,52 (+/-1,81) Punkten erreichen. Dabei konnten 14 der 25 Patienten (=56\%) dieser Gruppe mindestens ihren präoperativen Wert erreichen.

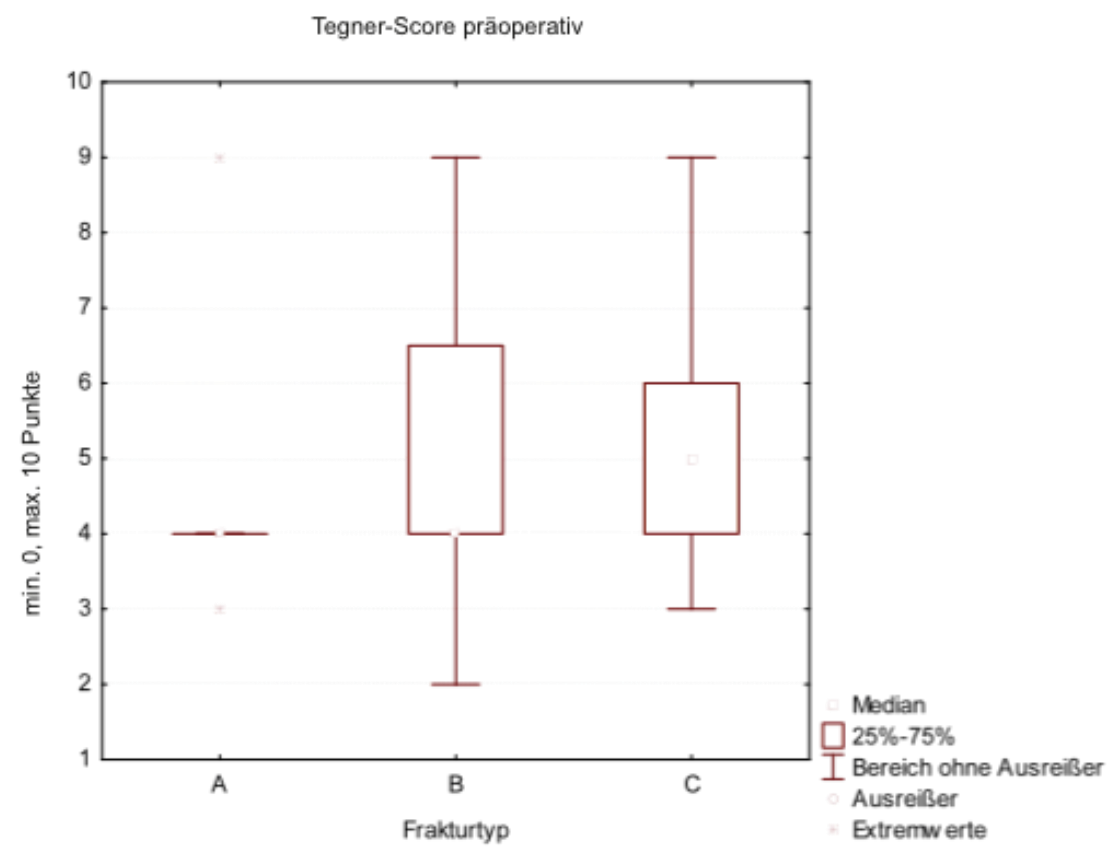

Abbildung 11: Darstellung der Ergebnisse im Tegner-Aktivitätsscore vor der Fraktur

\begin{tabular}{|c|c|c|c|}
\hline Frakturtyp & A & B & C \\
\hline Mittelwert & 4,57 & 4,93 & 5,28 \\
\hline Standardabweichung & 1,99 & 1,84 & 1,65 \\
\hline
\end{tabular}




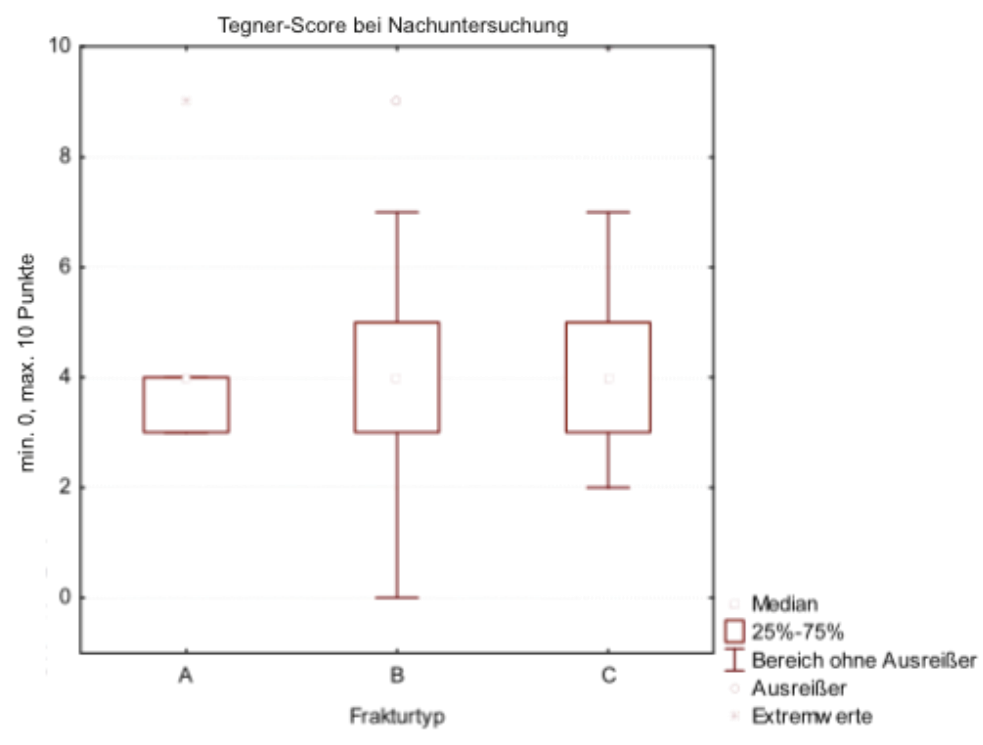

Abbildung 12: Darstellung der Ergebnisse im Tegner-Aktivitätsscore nach der Operation

\begin{tabular}{|c|c|c|c|}
\hline Frakturtyp & A & B & C \\
\hline Mittelwert & 4,43 & 4,07 & 4,52 \\
\hline Standardabweichung & 2,07 & 2,12 & 1,81 \\
\hline
\end{tabular}

Tabelle 12: Überblick über die Ergebnisse im Tegner-Aktivitätsscore nach der Operation

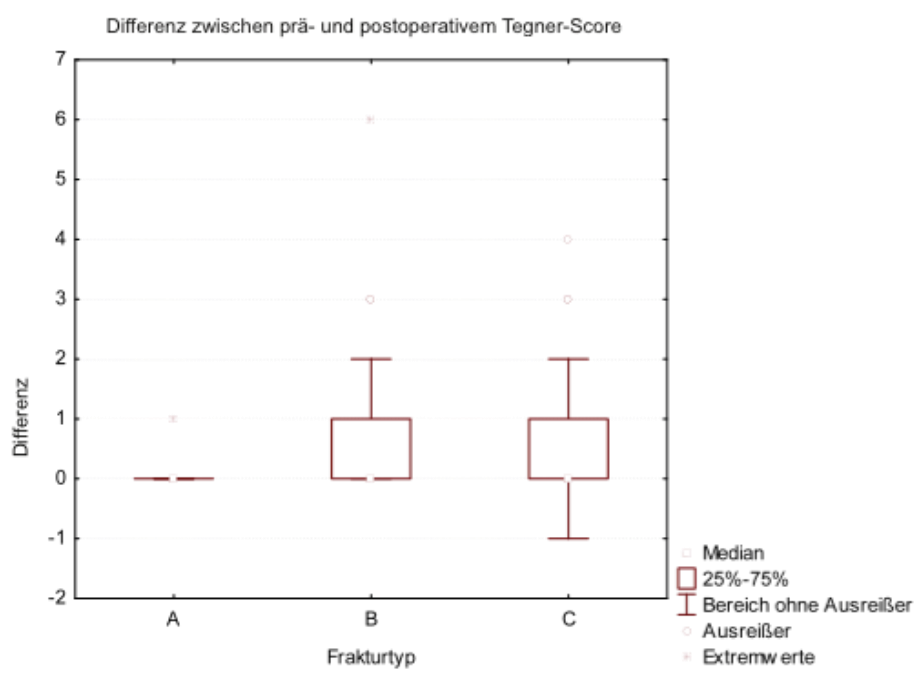

Abbildung 13: Darstellung der Differenz im Tegner-Aktivitätsscore prä- zu postoperativ 


\begin{tabular}{|c|c|c|c|}
\hline Frakturtyp & A & B & C \\
\hline Mittelwert & 0,14 & 0,86 & 0,76 \\
\hline Standardabweichung & 0.38 & 1,35 & 1,23 \\
\hline
\end{tabular}

Tabelle 13: Überblick über die Differenz im Tegner-Aktivitätsscore zwischen den präoperativen Angaben und der klinischen Nachuntersuchung

Signifikante Unterschiede im Tegner-Aktivitätsscore konnten weder bei den präoperativen Angaben $(p=0,306)$, bei den postoperativen Werten $(p=0,751)$ oder aus den Differenzen der vorher genannten $(p=0,317)$ ermittelt werden.

\subsubsection{WOMAC-Score}

Im WOMAC-Score erreichten die Patienten der Gruppe A minimal 0 und maximal 21 Punkte. Dabei betrug der durchschnittliche Wert der Patienten insgesamt 18,43 (+/- 23,55) Punkte und im WOMAC-Score-Gesamtindex wurde für die Gruppe A ein Wert von 0,73 $(+/-1,15)$ Punkten ermittelt.

In der Gruppe B wurden durchschnittlich 41,86 (+/- 50,99) Punkte erzielt. Dabei variierten die Werte der Patienten von vier Mal null (16\%) bis maximal 172 Punkten.

Bezogen auf den WOMAC-Score-Gesamtindex konnten in der Gruppe B 1,64 (+/- 1,96) Punkte festgestellt werden.

Die Patienten der Gruppe C erreichten Werte die minimal null und maximal 135 Punkte betrugen. Dabei wurden im Mittel 26,96 (+/- 31,02) Punkte notiert und für den WOMACScore-Gesamtindex konnten 1,09 (+/- 1,17) Punkte errechnet werden. 


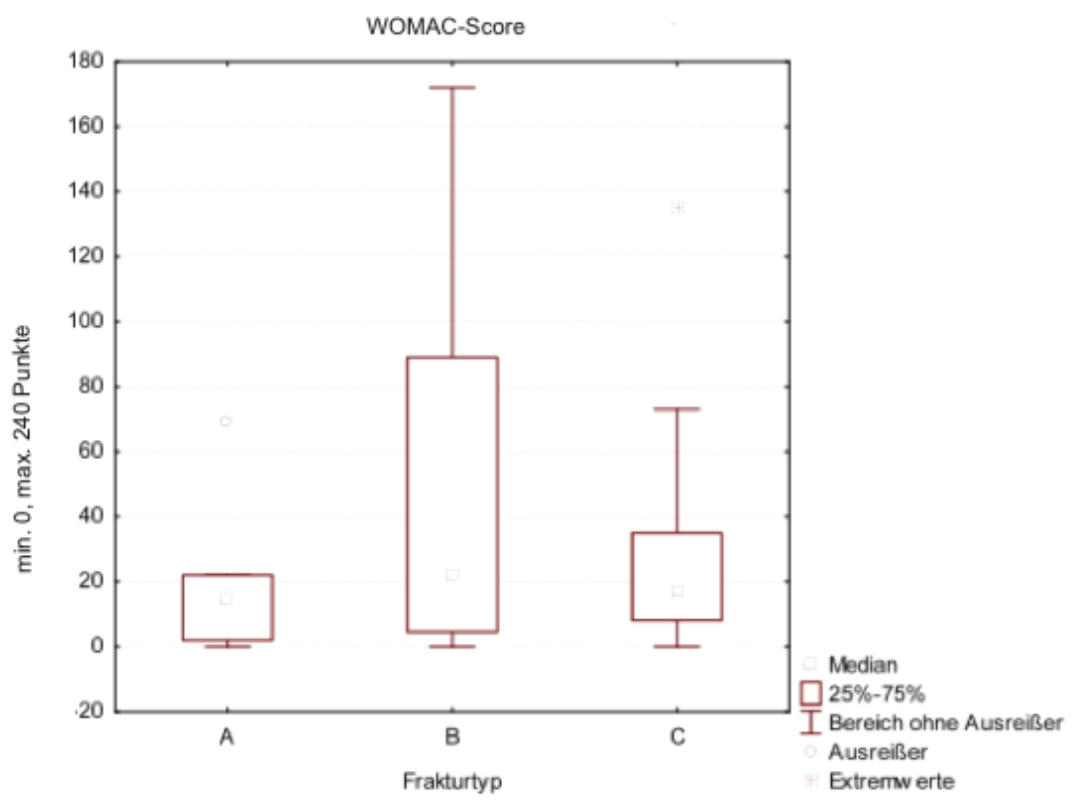

Abbildung 14: Darstellung der Ergebnisse im WOMAC-Score

\begin{tabular}{|c|c|c|c|}
\hline Frakturtyp & A & B & C \\
\hline Mittelwert & 18,43 & 41,86 & 26,96 \\
\hline Standardabweichung & 23,55 & 50,99 & 31,02 \\
\hline
\end{tabular}

Tabelle 14: : Überblick über die Ergebnisse im WOMC-Score

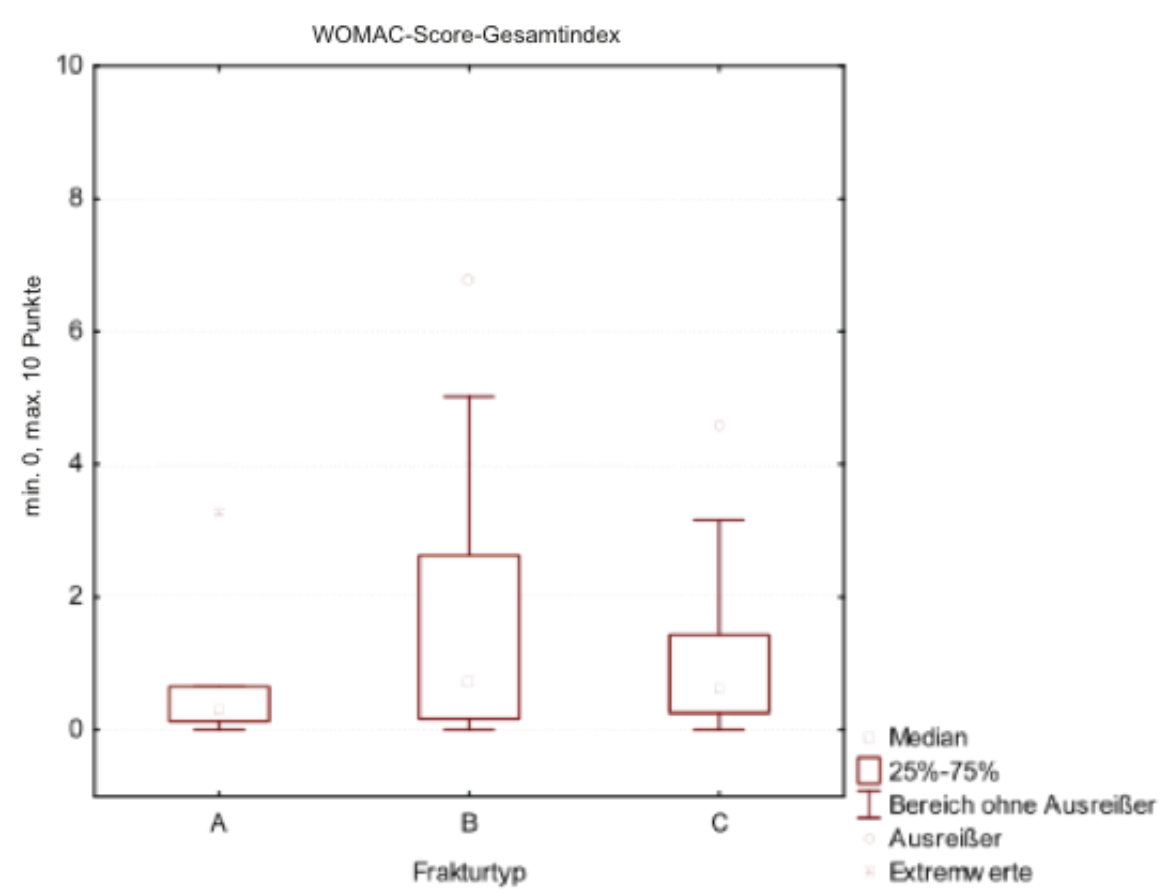

Abbildung 15: Darstellung der Ergebnisse des WOMAC-Score-Gesamtindexes 


\begin{tabular}{|c|c|c|c|}
\hline Frakturtyp & A & B & C \\
\hline Mittelwert & 0,73 & 1,64 & 1,09 \\
\hline Standardabweichung & 1,15 & 1,96 & 1,17 \\
\hline
\end{tabular}

Tabelle 15: Überblick über die Ergebnisse im WOMC-Score-Gesamtindex

Die Irrtumswahrscheinlichkeit des WOMAC-Scores liegt bei 0,693 und auch die Resultate des WOMAC-Score-Gesamtindexes ergaben eine Irrtumswahrscheinlichkeit von 0,511, weshalb beide Ergebnisse als nicht signifikant gewertet wurden.

\begin{tabular}{|c|c|c|c|}
\hline & Gruppe A & Gruppe B & Gruppe C \\
\hline $\begin{array}{c}\text { Exzellent } \\
\text { 0 - 12 Punkte }\end{array}$ & $4(57 \%)$ & $14(50 \%)$ & $8(32 \%)$ \\
\hline $\begin{array}{c}\text { Gut } \\
\mathbf{1 3}-\mathbf{3 8} \text { Punkte }\end{array}$ & $3(43 \%)$ & $4(14 \%)$ & $11(44 \%)$ \\
\hline $\begin{array}{c}\text { Zufriedenstellend } \\
\mathbf{3 9}-\mathbf{8 4} \text { Punkte }\end{array}$ & $0(0 \%)$ & $4(14 \%)$ & $4(16 \%)$ \\
\hline $\begin{array}{c}\text { Unbefriedigend } \\
\mathbf{8 5}-\mathbf{2 4 0} \text { Punkte }\end{array}$ & $0(0 \%)$ & $6(22 \%)$ & $2(8 \%)$ \\
\hline
\end{tabular}

Tabelle 16: Überblick über die Verteilung im WOMAC-Score

\subsubsection{Lysholm-Score}

Die Patienten der Gruppe A erreichten im Lysholm-Score minimal 64 und maximal 96 Punkte.

Ein Patient konnte ein exzellentes Ergebnis erzielen, fünf Patienten erreichten ein gutes Ergebnis und nur ein Ergebnis wurde mit 64 Punkten als unbefriedigend eingestuft. Dabei konnte die Gruppe im Mittel 86,86 (+/- 10,68) Punkte erzielen.

In der Gruppe B erreichten die Patienten durchschnittlich ein Ergebnis von 74,75 (+/- 20,19) Punkten, welches sich aus einem Wertebereich von minimal 23 und maximal 100 Punkten errechnen ließ.

Fünf Patienten erreichten ein exzellentes Ergebnis im WOMAC-Score, wobei zwei Patienten sogar die maximale Punktzahl von 100 erhielten. Sechs Patienten erreichten ein gutes Ergebnis und lagen in einem Wertebereich von 84 bis 90 Punkten. 
Bei zehn Patienten konnten ein zufriedenstellendes Ergebnis erzielt werden mit Ergebnissen zwischen 71 und 82 Punkten. Sieben Patienten erzielten ein unbefriedigendes Resultat. Ihre Ergebnisse lagen in einem Wertebereich zwischen 23 und 55 Punkten.

Die Patienten der Gruppe C erzielten im Lysholm-Score durchschnittlich 82,72 (+/- 17,33) Punkte. Das minimale Ergebnis lag in der Gruppe bei 23 Punkten und maximal wurde ein Wert von 100 Punkten erreicht. Sieben Patienten konnten dabei ein exzellentes Ergebnis erzielen.

Drei dieser sieben Patienten verzeichneten die maximale Punktzahl von 100. Ein insgesamt gutes Ergebnis konnte bei acht Patienten notiert werden.

Ihr Wertebereich setzte sich aus Ergebnissen zwischen 86 und 94 Punkten zusammen. In einem zufriedenstellenden Bereich wurden sieben Patienten eingestuft.

Ihre Werte variierten zwischen 66 und 83 Punkten. Bei drei Patienten, deren Werte minimal 33 und maximal 63 Punkte betrugen, wurde ein unbefriedigendes Ergebnis notiert.

\begin{tabular}{|c|c|c|c|}
\hline & Gruppe A & Gruppe B & Gruppe C \\
\hline $\begin{array}{c}\text { Exzellent } \\
95-100 \text { Punkte }\end{array}$ & $1(14 \%)$ & $5(18 \%)$ & $7(28 \%)$ \\
\hline $\begin{array}{c}\text { Gut } \\
94-84 \text { Punkte }\end{array}$ & $5(71 \%)$ & $6(21 \%)$ & $8(32 \%)$ \\
\hline $\begin{array}{c}\text { Zufriedenstellend } \\
83-65 \text { Punkte }\end{array}$ & $0(\%)$ & $10(36 \%)$ & $7(28 \%)$ \\
\hline $\begin{array}{c}\text { Unbefriedigend } \\
0-64 \text { Punkte }\end{array}$ & $1(14 \%)$ & $7(25 \%)$ & $3(12 \%)$ \\
\hline
\end{tabular}

Tabelle 17: Überblick über die Verteilung im Lysholm-Score 


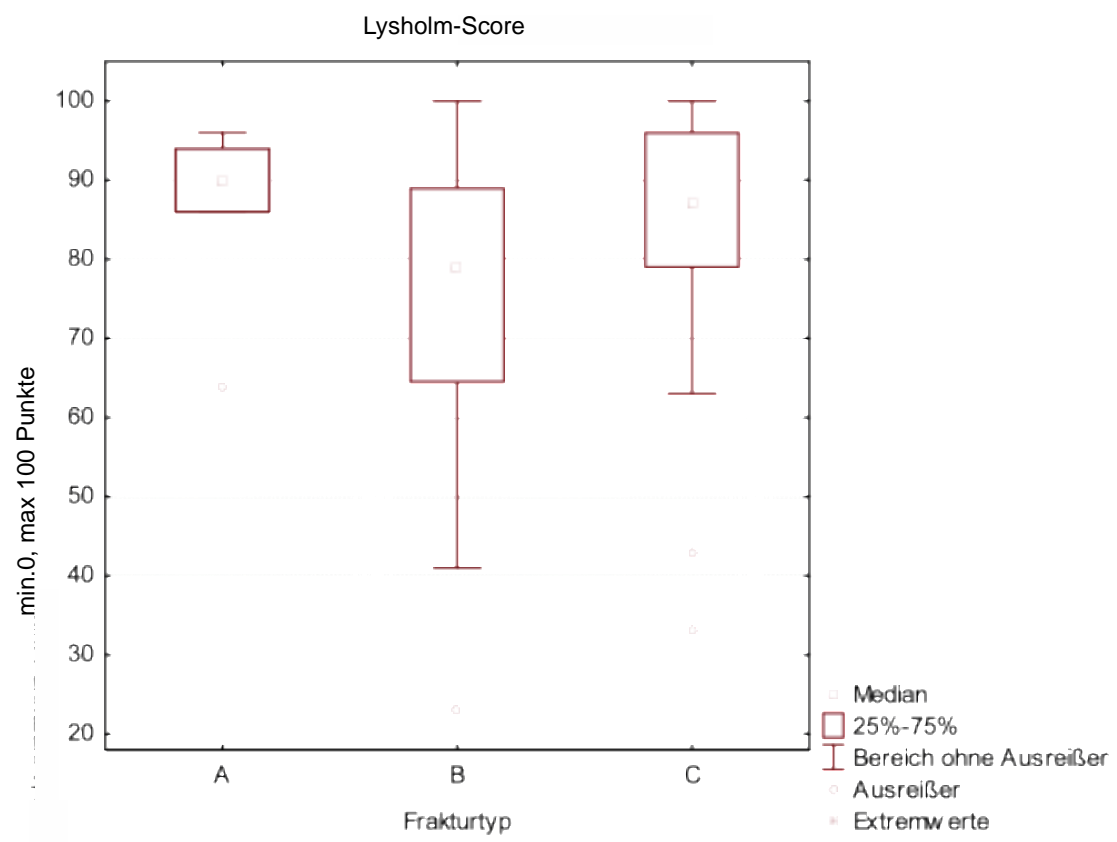

Abbildung 16: Darstellung der Ergebnisse im Lysholm-Score

\begin{tabular}{|c|c|c|c|}
\hline Frakturtyp & A & B & C \\
\hline Mittelwert & 86,86 & 74,75 & 82,72 \\
\hline Standardabweichung & 10,68 & 20,19 & 17,33 \\
\hline
\end{tabular}

Tabelle 18: Überblick über die Ergebnisse im Lysholm-Score

Zwischen den drei Frakturtypen konnte nach Untersuchung der Ergebnisse des LysholmScores zum Zeitpunkt der jeweiligen Nachuntersuchung kein signifikanter Unterschied $(\mathrm{p}=$ $0,114)$ festgestellt werden.

\subsection{Patientenzufriedenheit}

Sechs der sieben Patienten der Gruppe A erreichten ein exzellentes Ergebnis, worunter sich fünf Patienten mit der maximalen Punktzahl von zehn befanden. Nur ein Patient konnte ein unbefriedigendes Ergebnis von einem Punkt erzielen.

Die subjektive Zufriedenheit der Patienten betrug im Mittel 8,57 (+/- 3,36) der maximal erreichbaren zehn Punkte.

In der Gruppe B wies das Ergebnis bei 19 von 28 Patienten ein exzellentes Resultat auf. Zwölf der 19 Patienten konnten ein maximales Ergebnis von 10 Punkten erreichen. Vier Patienten mit einem Wertebereich von sechs und sieben Punkten erzielten ein 
zufriedenstellendes Ergebnis. Bei fünf Patienten wurde ein unbefriedigendes Ergebnis notiert. Es fand sich unter innen ein Wertebereich von minimal zwei bis maximal fünf Punkten. Somit konnte ein durchschnittlicher Wert von 8,32 (+/- 2,19) Punkten für die Patienten der Gruppe B notiert werden.

In der Gruppe C konnten zwölf der 13 Patienten mit einem exzellenten Ergebnis die maximal erreichbare Punktzahl erzielen. Bei sechs Patienten wurde ein gutes Ergebnis von acht Punkten notiert. Drei Patienten erreichten ein zufriedenstellendes Ergebnis von sechs oder sieben Punkten und drei Patienten wurde ein unbefriedigendes Ergebnis aufgeschrieben, deren Werte minimal drei und maximal fünf Punkte betrugen. Im Mittel ergab sich für die Gruppe C bei der subjektiven Patientenzufriedenheit ein Wert von 8,44 (+/- 1,94) Punkten.

\begin{tabular}{|c|c|c|c|}
\hline & Gruppe A & Gruppe B & Gruppe C \\
\hline $\begin{array}{c}\text { Exzellent } \\
\text { - 10 Punkte }\end{array}$ & $6(86 \%)$ & $19(68 \%)$ & $13(52 \%)$ \\
\hline $\begin{array}{c}\text { Gut } \\
\text { 8 Punkte }\end{array}$ & $0(0 \%)$ & $0(0 \%)$ & $6(24 \%)$ \\
\hline $\begin{array}{c}\text { Zufriedenstellend } \\
\text { 7 - 6 Punkte }\end{array}$ & $0(\%)$ & $4(14 \%)$ & $3(12 \%)$ \\
\hline $\begin{array}{c}\text { Unbefriedigend } \\
\text { 0- 5 Punkte }\end{array}$ & $1(14 \%)$ & $5(18 \%)$ & $3(12 \%)$ \\
\hline
\end{tabular}

Tabelle 19: Übersicht über die Angaben bei der Patientenzufriedenheit

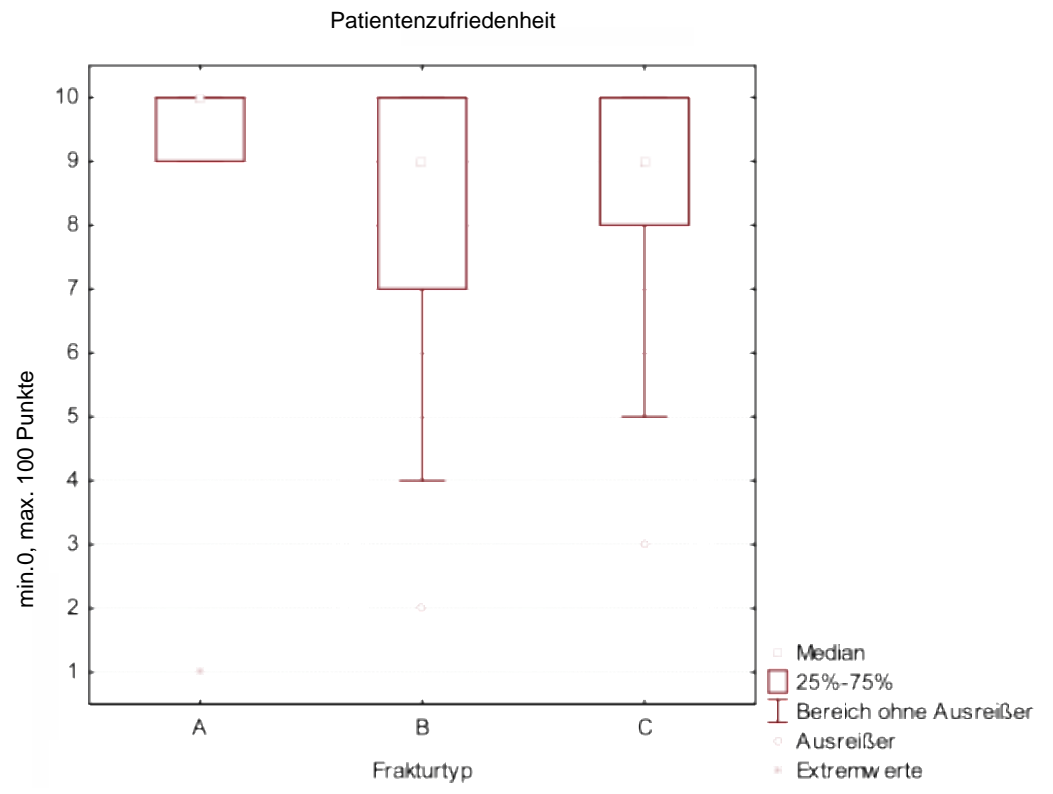

Abbildung 17: Darstellung der Ergebnisse der Patientenzufriedenheit 


\begin{tabular}{|c|c|c|c|}
\hline Frakturtyp & A & B & C \\
\hline Mittelwert & 8,57 & 8,32 & 8,44 \\
\hline Standardabweichung & 3,36 & 2,19 & 1,94 \\
\hline
\end{tabular}

Tabelle 20: Überblick über die Ergebnisse der Patientenzufriedenheit

Die Irrtumswahrscheinlichkeit bei der Patientenzufriedenheit war nicht signifikant mit $p=$ 0,544 .

\subsection{Radiologischer Bewertungsbogen}

Unter den 60 Patienten wiesen 13 (22\%) Teilnehmer eine radiologisch nachweisbare Stufe in den postoperativ durchgeführten Röntgenaufnahmen auf.

Unter den Patienten in unserer Studie mit einer verbliebenen Gelenkstufe (retropatellares Gleitlager) fanden sich sechs Patienten (46\%) mit einer Quer- und sieben Patienten (54\%) mit einer Trümmerfraktur. Drei Patienten wiesen eine Stufe kleiner als ein Millimeter auf, neun Patienten hatten eine Stufe zwischen einem und drei Millimeter und ein Patient wies eine Stufe größer als drei Millimeter auf.

\subsubsection{Visuell numerische Analogskala Schmerz}

In der visuell numerischen Analogskala Schmerz erreichten die Patienten ohne Auffälligkeiten durchschnittlich $1,77(+/-2,14)$ und die Patienten mit einer Stufe 1,54 (+/1,61) Punkte.

Die Unterschiede in der visuell numerischen Analogskala der zwei Patientenkollektive waren nicht signifikant zueinander $(p=0,641)$. 


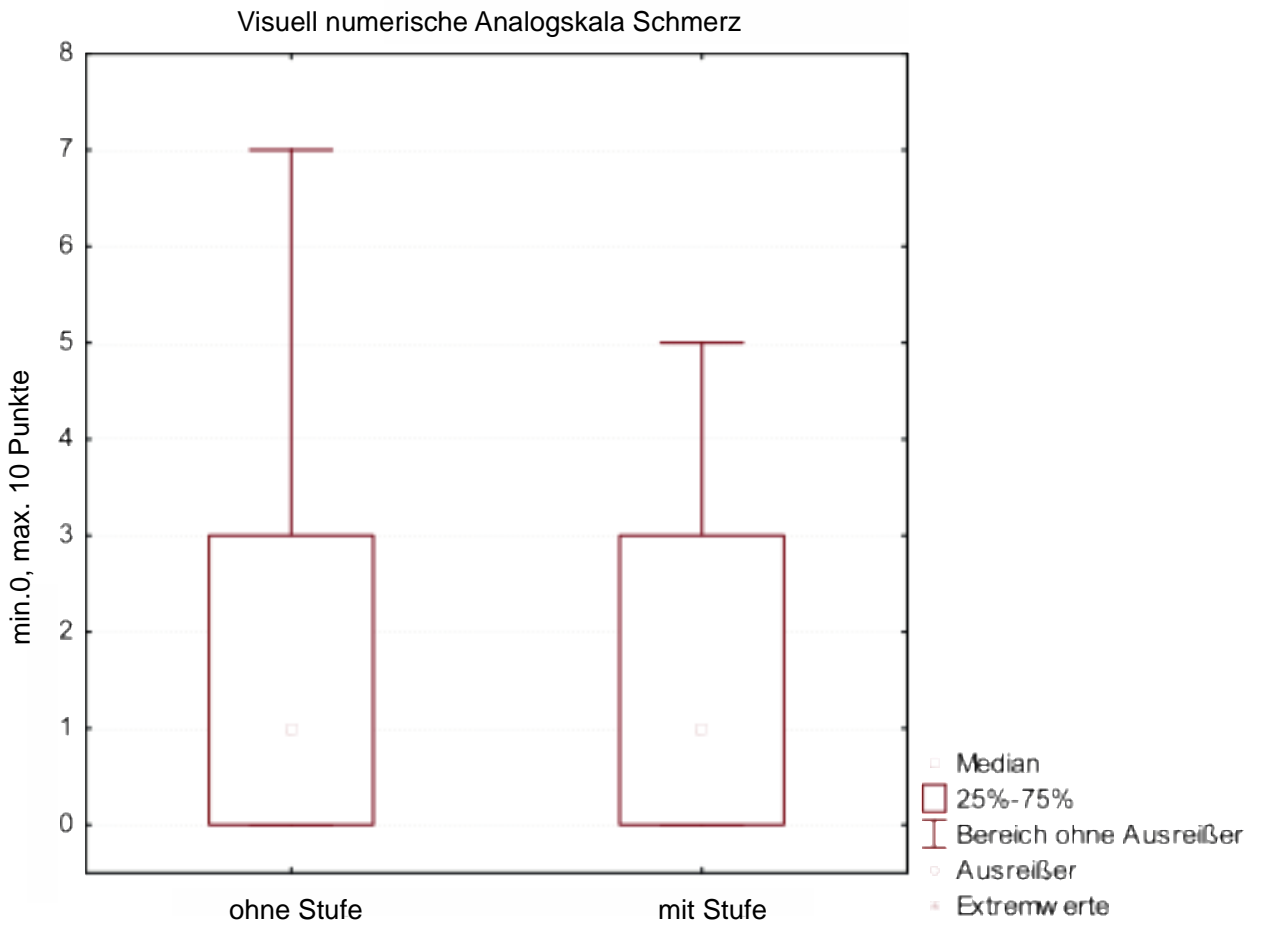

Abbildung 18: Darstellung der Ergebnisse der visuell numerischen Analogskala Schmerz

\begin{tabular}{|l|l|l|}
\hline & Ohne Stufe & Mit Stufe \\
\hline Mittelwert & 1,77 & 1,54 \\
\hline Standardabweichung & 2,14 & 1,61 \\
\hline
\end{tabular}

Tabelle 21: Überblick über die Ergebnisse in der visuell numerischen Analogskala Schmerz

\subsubsection{American Knee Society Score}

In dem AKSS erzielten die Patienten ohne Auffälligkeiten im Mittel 168,53 (+/- 29,56) Punkte. Die Patienten mit einer Stufe erreichten durchschnittlich 161,15 (+/- 34,57) Punkte, weshalb die Ergebnisse im AKSS nicht signifikant zueinander $(p=0,478)$ waren. 


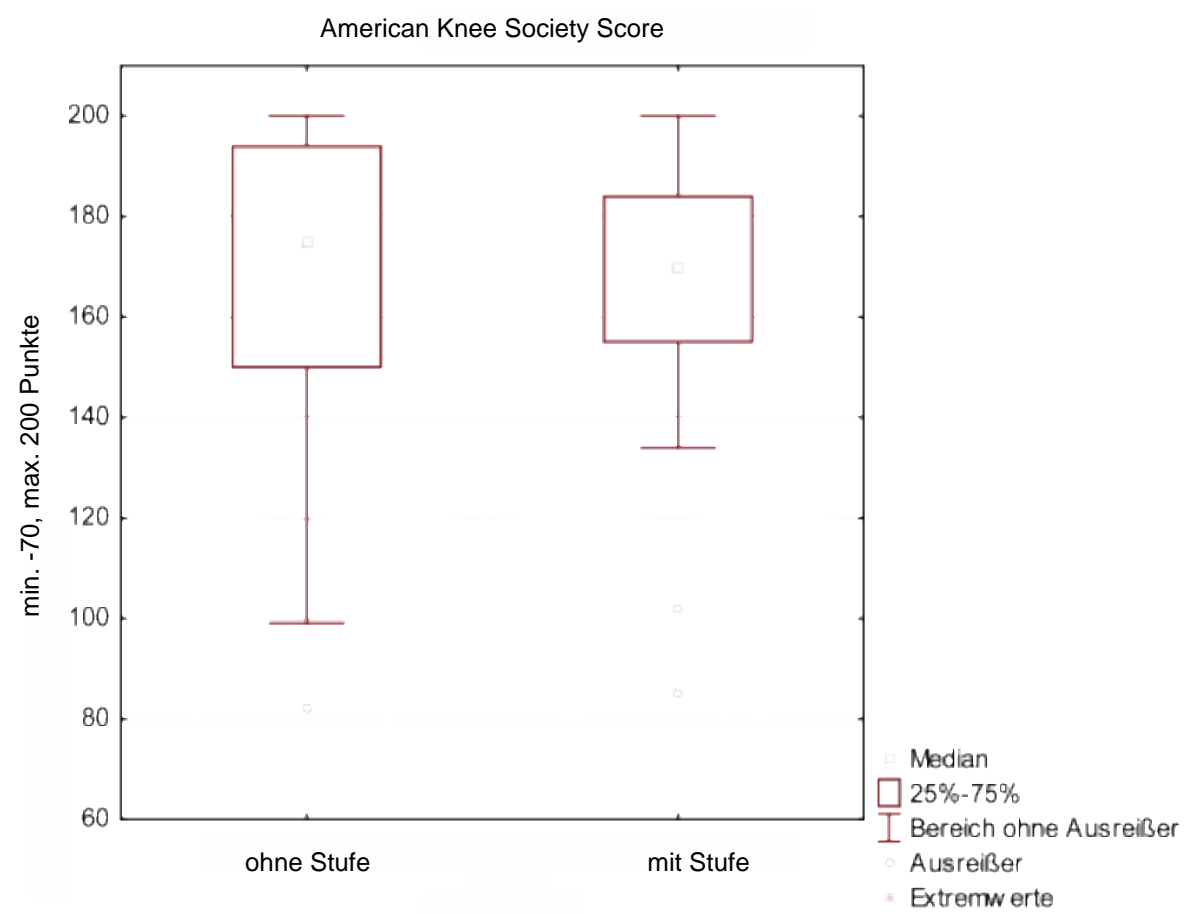

Abbildung 19: Darstellung der Ergebnisse des American Knee Society Scores

\begin{tabular}{|l|l|l|}
\hline & Ohne Stufe & Mit Stufe \\
\hline Mittelwert & 168,53 & 161,15 \\
\hline Standardabweichung & 29,56 & 34,57 \\
\hline
\end{tabular}

Tabelle 22: Überblick über die Ergebnisse im American Knee Society Scores

\subsubsection{Tegner-Aktivitätsscore präoperativ}

Die Patienten ohne eine radiologische Auffälligkeit erzielten im Mittel im TegnerAktivitätsscore präoperativ 5,09 (+/- 1,82) Punkte. 4,85 (+/- 1,63) Punkte wurden durchschnittlich in der Gruppe von Patienten mit radiologischen Auffälligkeiten erreicht.

Die Unterschiede in den präoperativen Angaben der Patientenkollektive waren nicht signifikant zueinander $(p=0,992)$. 


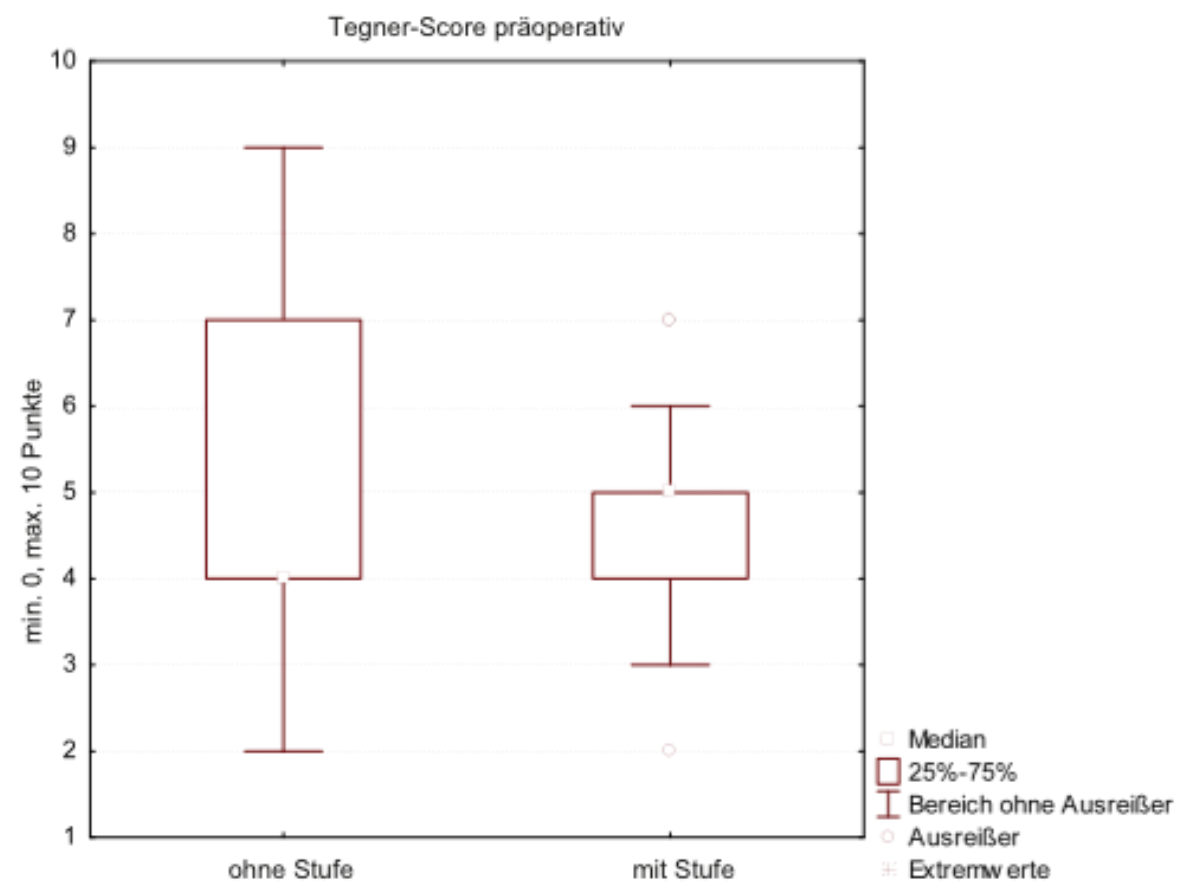

Abbildung 20: Darstellung der Ergebnisse des präoperativen Tegner-Aktivitätsscores

\begin{tabular}{|l|l|l|}
\hline & Ohne Stufe & Mit Stufe \\
\hline Mittelwert & 5,09 & 4,85 \\
\hline Standardabweichung & 1,82 & 1,63 \\
\hline
\end{tabular}

Tabelle 23: Überblick über die Ergebnisse des präoperativen Tegner-Aktivitätsscores

\subsubsection{Tegner-Aktivitätsscore postoperativ}

Postoperativ ereichten die Patienten ohne radiologische Auffälligkeiten durchschnittlich 4,04 $(+/-1,99)$ Punkte und für die Patienten mit radiologischen Auffälligkeiten wurden im Mittel 3,92 (+/- 1,93) Punkte notiert.

Die Unterschiede in den postoperativen Resultaten der Patientenkollektive waren nicht signifikant zueinander $(p=0,712)$. 


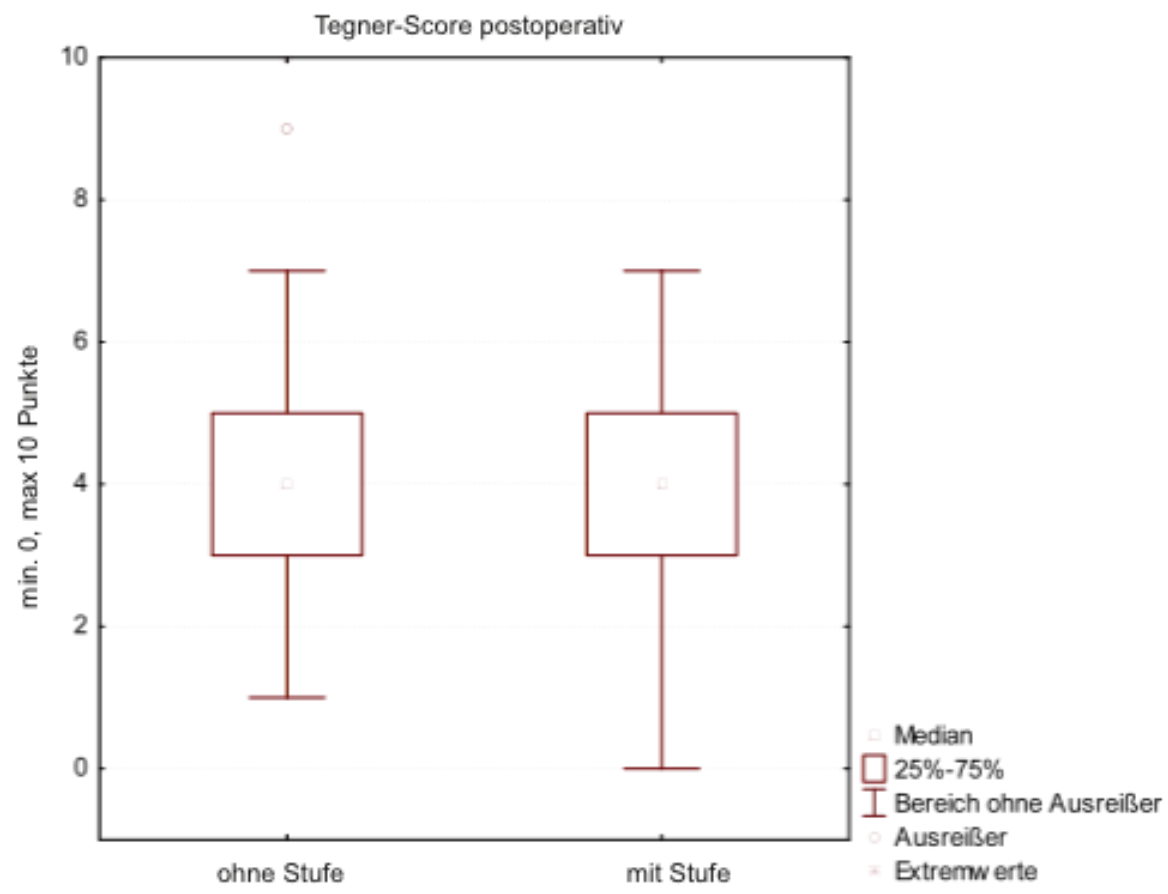

Abbildung 21: Darstellung der Ergebnisse des postoperativen Tegner-Aktivitätsscores

\begin{tabular}{|l|l|l|}
\hline & Ohne Stufe & Mit Stufe \\
\hline Mittelwert & 4,04 & 3,92 \\
\hline Standardabweichung & 1,99 & 1,93 \\
\hline
\end{tabular}

Tabelle 24: Überblick über die Ergebnisse des postoperativen Tegner-Aktivitätsscores

\subsubsection{Differenz Tegner-Aktivitätsscore}

In der postoperativ ermittelten Differenz des Tegner-Aktivitätsscore wurde für die Patienten ohne radiologisches Korrelat eine Differenz von 0,68 (+/- 1,30) Punkten notiert. Bei den Patienten mit radiologischer Auffälligkeit wurde im Mittel eine Differenz von 0,92 (+/- 0,95) Punkten bestimmt.

Die Differenzen im Tegner-Aktivitätsscore der Patientenkollektive waren nicht signifikant zueinander $(p=0,293)$. 


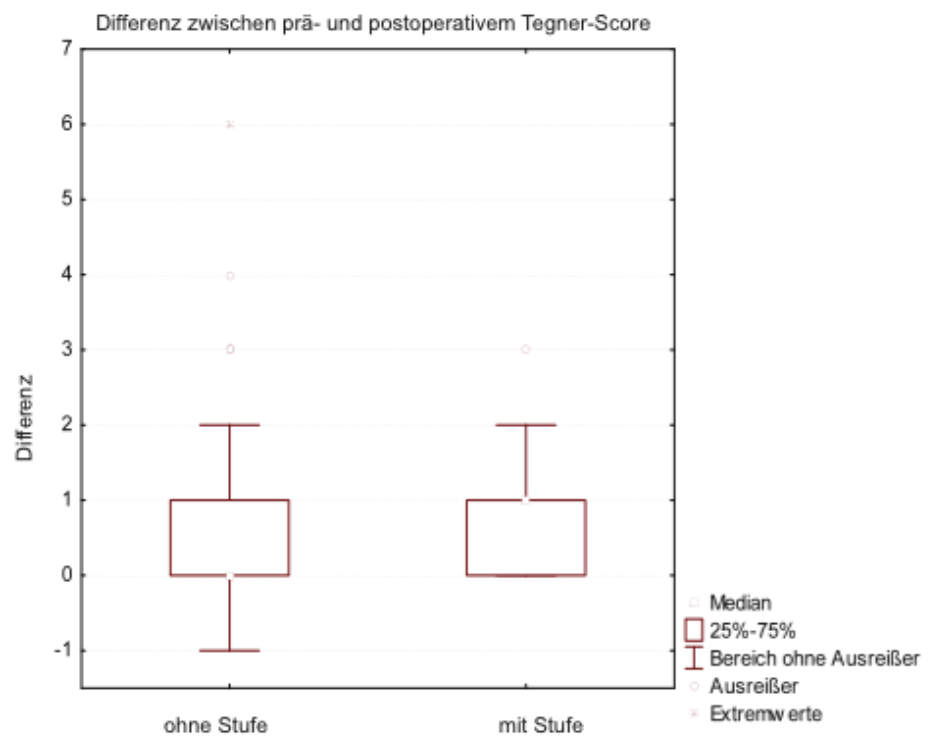

Abbildung 22: Darstellung der Differenz im Tegner-Aktivitätsscore

\begin{tabular}{|l|l|l|}
\hline & Ohne Stufe & Mit Stufe \\
\hline Mittelwert & 0,68 & 0,92 \\
\hline Standardabweichung & 1,30 & 0,95 \\
\hline
\end{tabular}

Tabelle 25: Überblick über die Differenz des Tegner-Aktivitätsscores

\subsubsection{WOMAC-Score}

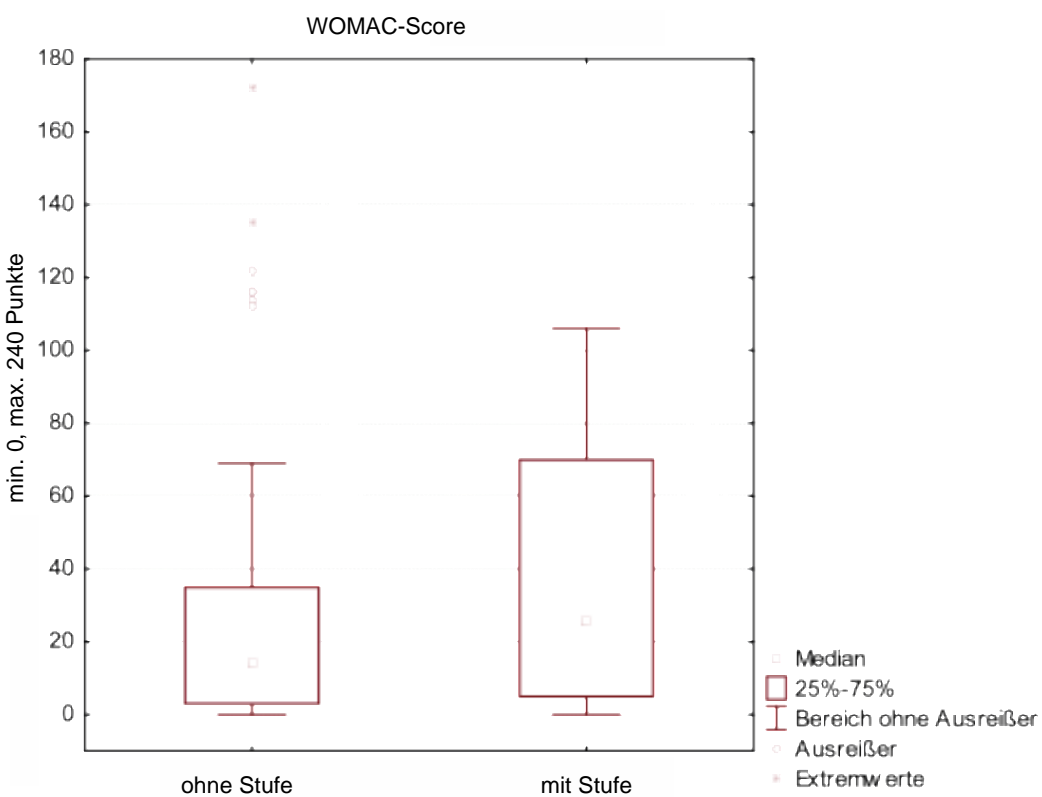

Abbildung 23: Darstellung der Ergebnisse des WOMAC-Scores 


\begin{tabular}{|l|l|l|}
\hline & Ohne Stufe & Mit Stufe \\
\hline Mittelwert & 33,08 & 32,31 \\
\hline Standardabweichung & 42,3 & 39,73 \\
\hline
\end{tabular}

Tabelle 26: Überblick über die Ergebnisse des WOMAC-Scores

Die Patienten ohne eine radiologische Auffälligkeit erzielten im WOMAC-Score im Mittel 33,08 (+/- 42,3) Punkte. Patienten mit einer Stufe erzielten durchschnittlich 32,31 (+/- 39,73) Punkte im WOMAC-Score.

Die Irrtumswahrscheinlichkeit im WOMAC-Score beträgt $p=0,472$.

\subsubsection{WOMAC-Score-Gesamtindex}

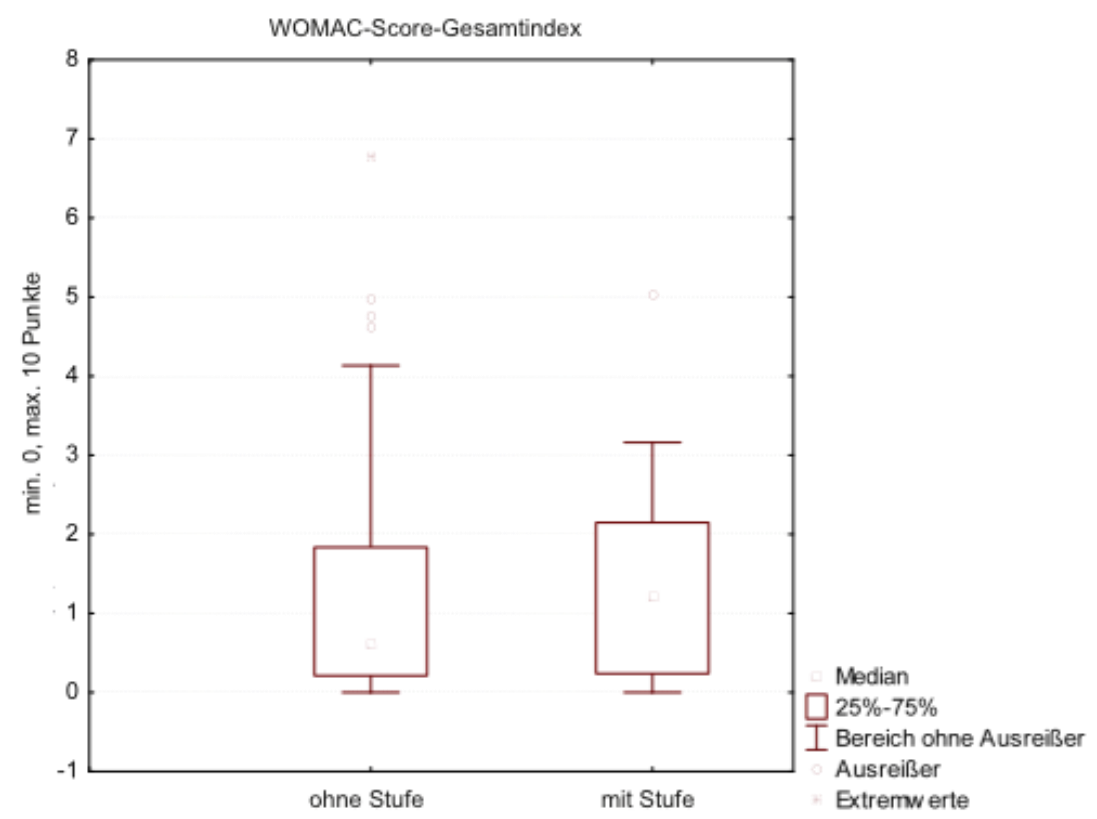

Abbildung 24: Darstellung der Ergebnisse des WOMAC-Score-Gesamtindexes

\begin{tabular}{|l|l|l|}
\hline & Ohne Stufe & Mit Stufe \\
\hline Mittelwert & 1,27 & 1,43 \\
\hline Standardabweichung & 1,64 & 1,49 \\
\hline
\end{tabular}

Tabelle 27: Überblick über die Ergebnisse des WOMAC-Score-Gesamtindexes 
Im WOMAC-Score-Gesamtindex wurde für die Patienten ohne radiologische Auffälligkeiten durchschnittlich 1,27 (+/- 1,64) Punkte notiert, wohingegen für die Patienten mit radiologischem Korrelat im Mittel ein Wert von 1,43 (+/- 1,49) erreicht wurde.

Die Ergebnisse im WOMAC-Score-Gesamtindex waren nicht signifikant zueinander $(\mathrm{p}=$ $0,634)$.

\subsubsection{Lysholm-Score}

In dem Lysholm-Score wurden für die Patienten ohne radiologische Auffälligkeiten ein Wert von 79,74 (+/- 19,61) Punkten notiert, wohingegen für die Patienten mit radiologischem Korrelat im Mittel ein Wert von 78,54 (+/- 14,32) erreicht wurde.

Die Unterschiede im Lysholm-Score der zwei Patientenkollektive waren nicht signifikant zueinander $(p=0,232)$.

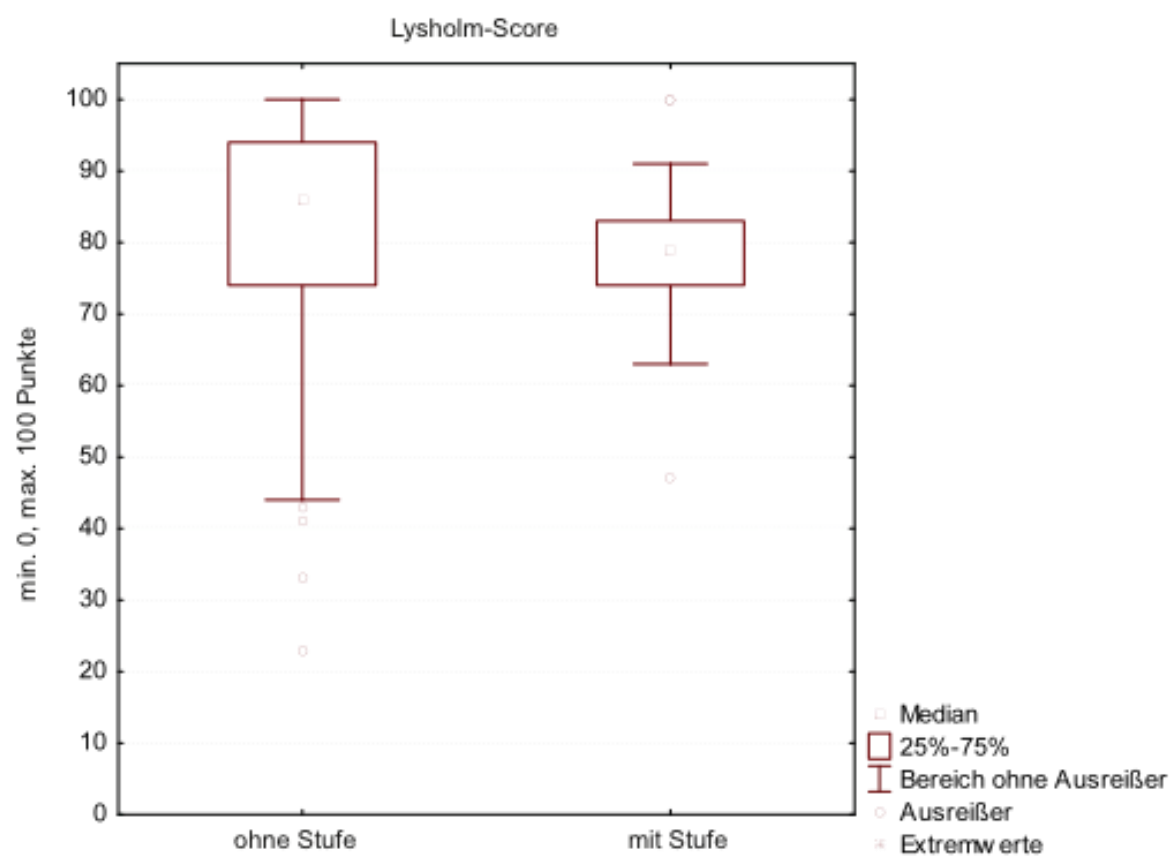

Abbildung 25: Darstellung der Ergebnisse des Lysholm-Scores

\begin{tabular}{|l|l|l|}
\hline & Ohne Stufe & Mit Stufe \\
\hline Mittelwert & 79,74 & 78,54 \\
\hline Standardabweichung & 19,61 & 14,32 \\
\hline
\end{tabular}

Tabelle 28: Überblick über die Ergebnisse des Lysholm-Scores 


\subsubsection{Patientenzufriedenheit}

Für die Patienten ohne radiologische Auffälligkeit wurde im Mittel ein Wert von 8,57 (+/- 2,23) Punkten festgehalten.

In der zweiten Gruppe konnten für die Patienten mit radiologischer Auffälligkeit durchschnittlich 7,77 (+/- 2,09) Punkte notiert werden.

Die Unterschiede in der Patientenzufriedenheit waren nicht signifikant zueinander $(p=0,686)$.

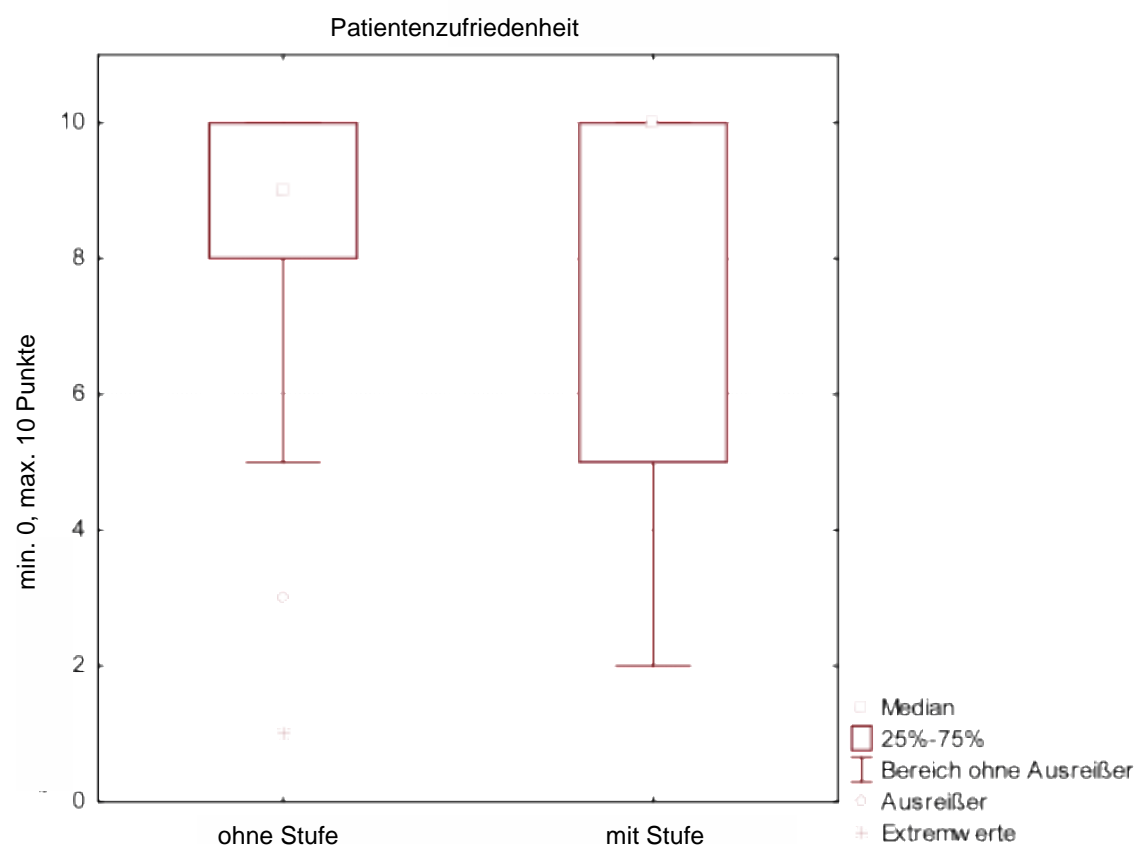

Abbildung 26: Darstellung der Ergebnisse der Patientenzufriedenheit

\begin{tabular}{|l|l|l|}
\hline & Ohne Stufe & Mit Stufe \\
\hline Mittelwert & 8,57 & 7,77 \\
\hline Standardabweichung & 2,23 & 2,09 \\
\hline
\end{tabular}

Tabelle 29: Überblick über die Ergebnisse der Patientenzufriedenheit 


\subsection{Vergleich der Ergebnisse von Patienten mit hyperdensen Arealen gegenüber Patienten ohne Auffälligkeiten}

Unter den 60 analysierten Patienten befanden sich 54 (90\%) ohne Nachweis auf ein hyperdenses Areal (nekrotischer Bereich), wohingegen bei sechs (10\%) eine Auffälligkeit erkannt wurde (siehe Röntgenscore des Ergebnisteils).

Unter den 54 Patienten (Gruppe 1 = keine Nekrose der Patella) waren 38 Männer und 16 Frauen, die im Mittel 45,83 (+/- 18,73; 11-84) Jahre alt waren und einen durchschnittlichen BMI von 24,64 (+/- 4,32; 16,97-40,09) kg/m² aufwiesen.

Die Patienten der Gruppe 1 wurden im Mittel 92,1 (+/- 104,4; 1-504) Stunden nach ihrer Verletzung (Zeitpunkt der Frakturentstehung) operiert und fanden sich durchschnittlich 63,45 (+/- 33,16; 19-136) Monate nach dieser Operation zur Nachuntersuchung in der Universitätsmedizin Göttingen ein.

Die sechs Patienten der Gruppe 2 (= Patienten mit nekrotischen Anteilen der Patella) setzten sich aus fünf Männern und einer Frau zusammen, die insgesamt einen Altersdurchschnitt von 42,33 (+/- 17,7; 19-64) Jahren aufwiesen und BMI im Mittel von 25,27 (+/- 2,30; 22,27 $28,35) \mathrm{kg} / \mathrm{m}^{2}$ besaßen.

Die Operation wurde im Mittel 124 (+/-201; 3-525) Stunden nach der

Frakturentstehung durchgeführt und die Nachuntersuchung im Klinikum fand im Mittel 95 (+/28,71; 46-133) Monate nach der Operation statt.

\subsubsection{Visuell numerische Analogskala Schmerz (VASS)}

In der visuellen numerischen Analogskala für Schmerzen gaben die Patienten ohne Nekrosen im Mittel 1,61 (+/- 1,89) Punkte an, die sich aus Werten von minimal null bis maximal sieben Punkten zusammensetzte.

Die Patienten mit einer Nekrose haben im Durchschnitt einen Wert von 2,66 (+/- 3,08) Punkten erzielt und wiesen minimal null und maximal sieben Punkte auf. 


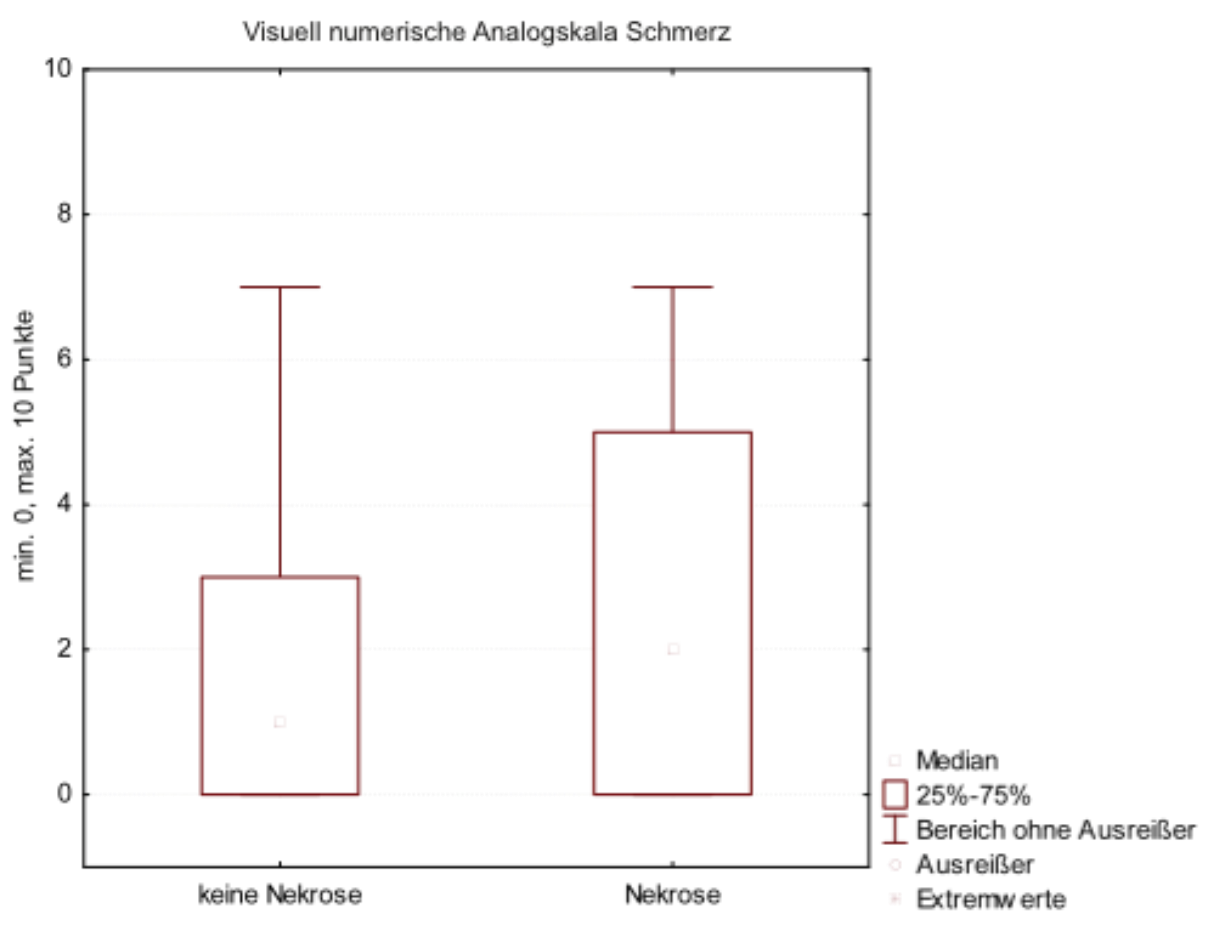

Abbildung 27: Darstellung der Ergebnisse von Patienten ohne Nekrose gegenüber Patienten mit Nekrose für die visuell numerische Analogskala Schmerz

\begin{tabular}{|c|c|c|}
\hline & Keine Nekrose & Nekrose \\
\hline Mittelwert & 1,61 & 2,66 \\
\hline Standardabweichung & 1,89 & 3,08 \\
\hline
\end{tabular}

Tabelle 30: Überblick über die Ergebnisse der visuell numerische Analogskala Schmerz

Zwischen den beiden Patientenkollektiven konnte nach Untersuchung der Ergebnisse der visuell numerischen Analogskala Schmerz zum Zeitpunkt der jeweiligen Nachuntersuchung kein signifikanter Unterschied festgestellt werden $(p=0,587)$.

\subsubsection{American Knee Society Score (AKSS)}

Im American Knee Society Score erzielten die Patienten der Gruppe 1 im Durchschnitt 167,5 (+/- 30,28) Punkte. Dabei wurden Werte von minimal 82 und der maximalen Punktzahl von 200 erreicht.

In der Gruppe 2 erreichten die Patienten im Mittel 161,83 (+/- 35,58) Punkte. Der Minimalwert lag bei 103 und der Maximalwert bei 195 Punkten. 


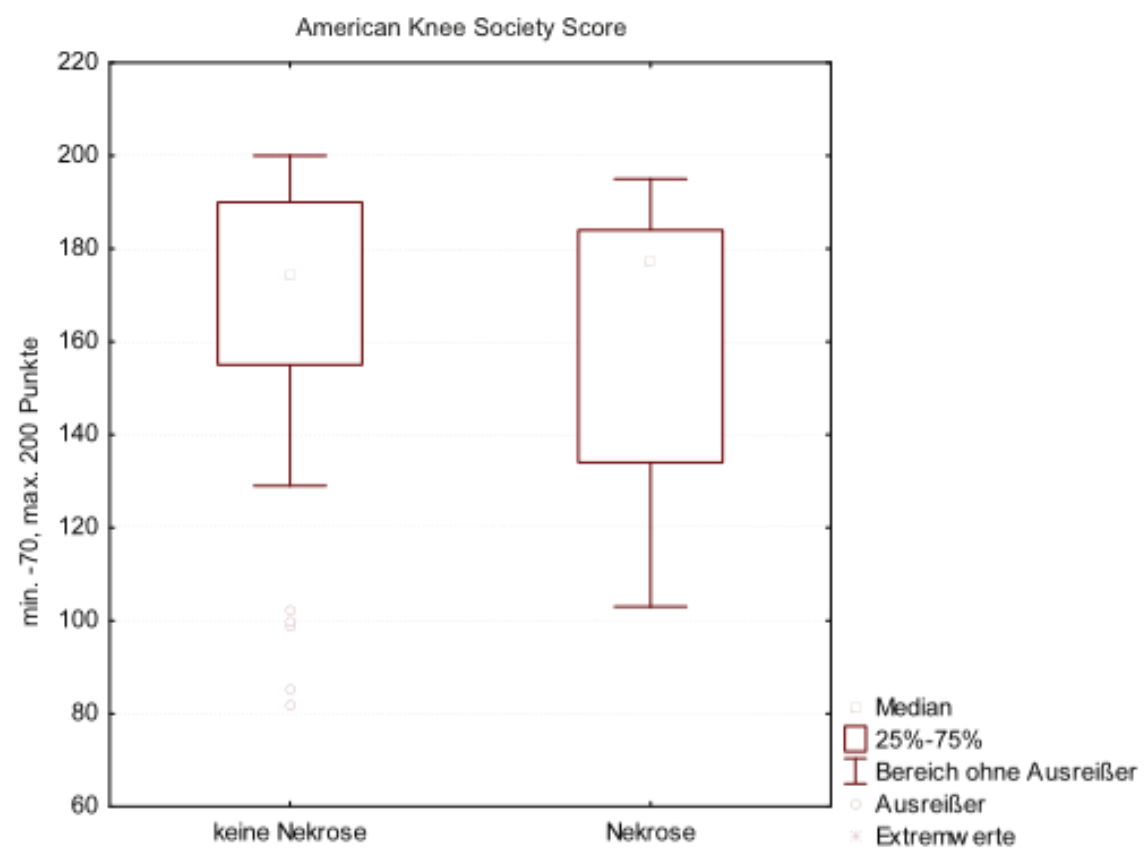

Abbildung 28: Darstellung der Ergebnisse von Patienten ohne Nekrose gegenüber Patienten mit Nekrose für den American Knee Society Score

Zwischen den beiden Patientenkollektiven konnte nach Untersuchung der Ergebnisse des American Knee Society Scores zum Zeitpunkt der Nachuntersuchung somit kein signifikanter Unterschied festgestellt werden $(p=0,805)$.

\begin{tabular}{|c|c|c|}
\hline & Keine Nekrose & Nekrose \\
\hline Mittelwert & 167,5 & 161,83 \\
\hline Standardabweichung & 30,28 & 35,58 \\
\hline
\end{tabular}

Tabelle 31: Überblick über die Ergebnisse des American Knee Society Scores

\subsubsection{Tegner-Aktivitätsscore (präoperativ)}

Vor der Operation erreichten die Patienten ohne radiologisch nachgewiesene Nekrose (Gruppe 1) im Tegner-Aktivitätsscore einen durchschnittlichern Wert von 5,04 (+/- 1,83) Punkten. Ihre Werte variierten von minimal zwei bis maximal neun der zu erreichenden zehn Punkte. 
Das Patientenkollektiv mit radiologisch gesicherten Nekrosen erreichte einen nahezu identischen Mittelwert von 5,00 (+/- 1,09) Punkten. Ihr Wertebereich umfasste Angaben von minimal vier bis maximal sieben Punkten.

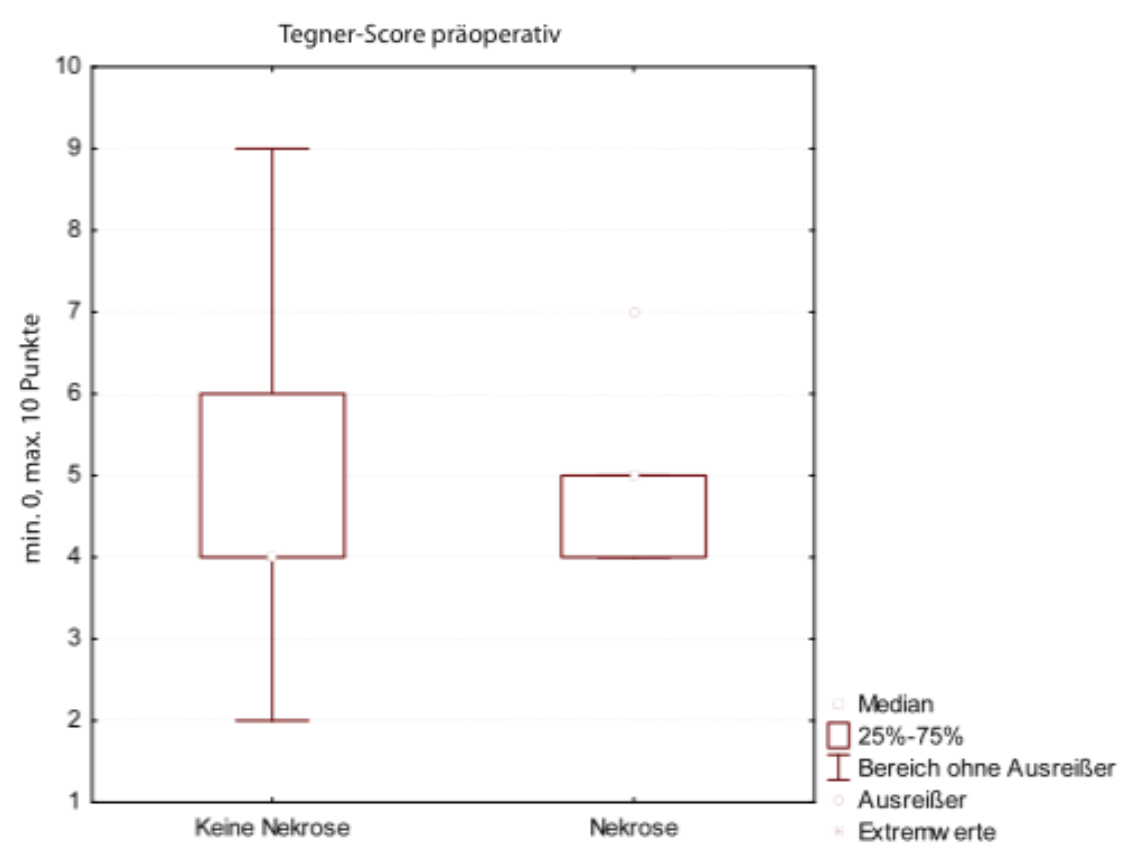

Abbildung 29: Darstellung der Ergebnisse von Patienten ohne Nekrose gegenüber Patienten mit Nekrose für den Tegner-Aktivitätsscore präoperativ

\begin{tabular}{|c|c|c|}
\hline & Keine Nekrose & Nekrose \\
\hline Mittelwert & 5,04 & 5,00 \\
\hline Standardabweichung & 1,83 & 1,09 \\
\hline
\end{tabular}

Tabelle 32: Überblick über die Ergebnisse des Tegner-Aktivitätsscores präoperativ

Die Irrtumswahrscheinlichkeit p des präoperativen Tegner-Aktivitätsscores liegt bei 0,6485.

\subsubsection{Tegner-Aktivitätsscore (postoperativ)}

Zum Zeitpunkt der Nachuntersuchung konnten für die Patienten der Gruppe 1 im Mittel 4,33 $(+/-2,06)$ Punkte festgestellt werden. Dabei wurden minimal null 0 und einmal die maximale Punktzahl von zehn erreicht.

Die Patienten der Gruppe 2 erzielten durchschnittlich einen etwas geringeren Wert von 4,00 $(+/-0,89)$ Punkten. Ihr durchschnittlicher Wert setzte sich aus Angaben von minimal drei bis maximal fünf Punkten zusammen. 


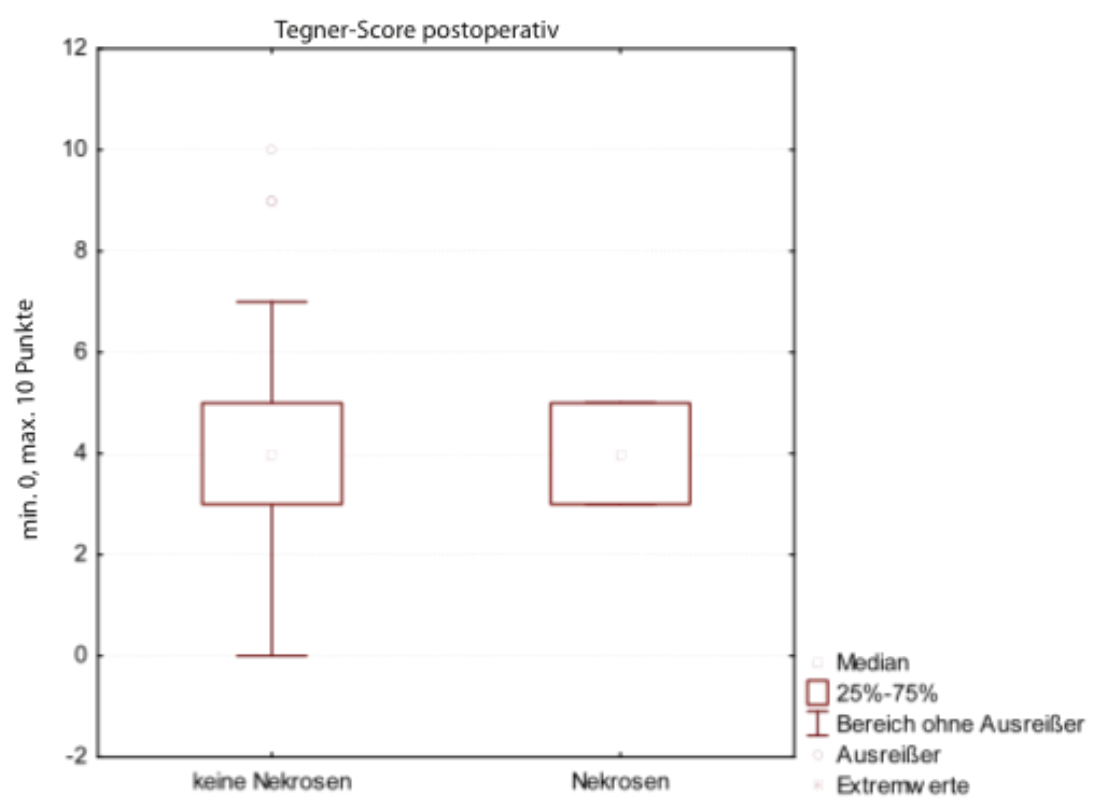

Abbildung 30: Darstellung der Ergebnisse von Patienten ohne Nekrose gegenüber Patienten mit Nekrose für den Tegner-Aktivitätsscore postoperativ

\begin{tabular}{|c|c|c|}
\hline & Keine Nekrose & Nekrose \\
\hline Mittelwert & 4,33 & 4,00 \\
\hline Standardabweichung & 2,06 & 0,89 \\
\hline
\end{tabular}

Tabelle 33: Überblick über die Ergebnisse des Tegner-Aktivitätsscores postoperativ

Die Ergebnisse im postoperativ ermittelten Tegner-Aktivitätsscore waren nicht signifikant zueinander $(p=0,970)$. 


\subsubsection{Differenz im Tegner-Aktivitätsscore (prä- zu postoperativ)}

Die Differenz zwischen den Ergebnissen der präoperativen Angaben und derer zum Zeitpunkt der klinischen Nachuntersuchung waren in der Gruppe 2 etwas größer als in Gruppe 1.

So verminderte sich die Punktzahl in Gruppe 1 im Mittel um 0,7 (+/- 1,24) Punkte und in Gruppe 2 war mit durchschnittlich 1,0 (+/- 1,26) Punkten eine größere Differenz feststellbar.

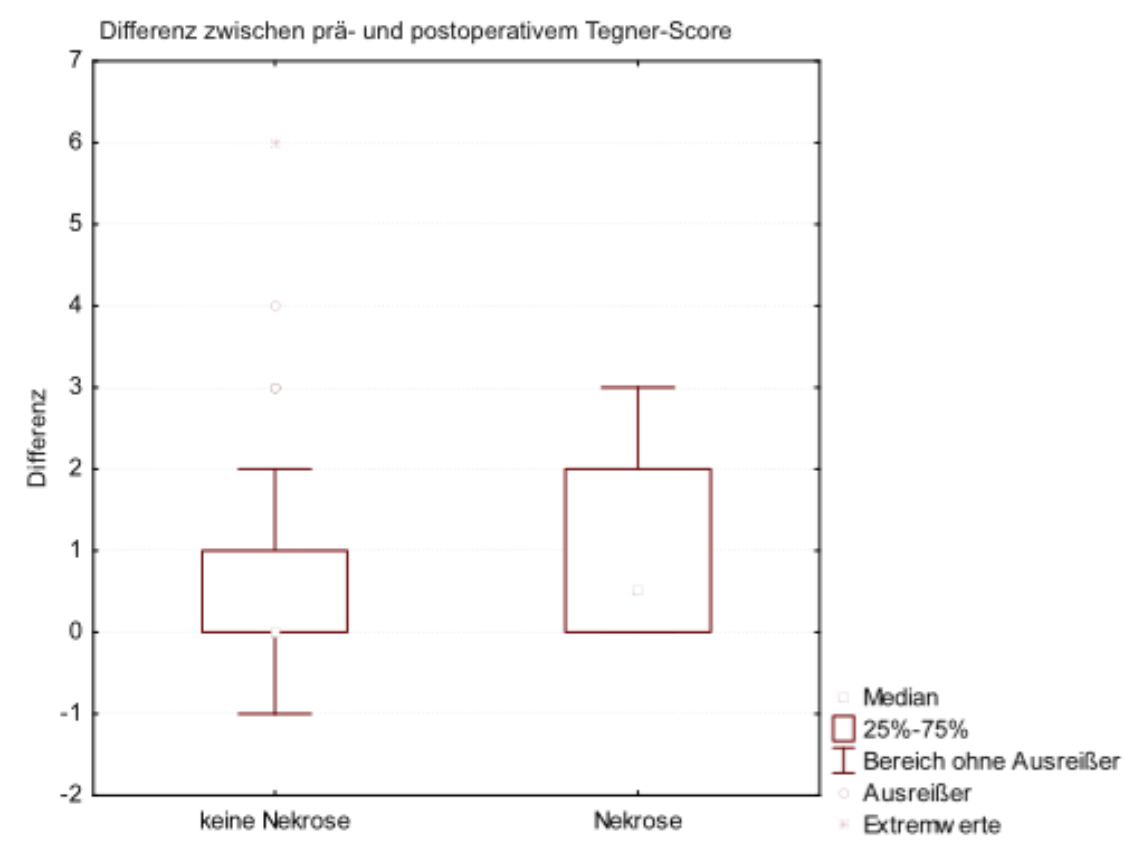

Abbildung 31: Darstellung der Differenz der Ergebnisse von Patienten ohne Nekrose gegenüber Patienten mit Nekrose für den Tegner-Aktivitätsscore postoperativ

\begin{tabular}{|c|c|c|}
\hline & Keine Nekrose & Nekrose \\
\hline Mittelwert & 0,7 & 1,0 \\
\hline Standardabweichung & 1,24 & 1,26 \\
\hline
\end{tabular}

Tabelle 34: Überblick über die Differenz im Tegner-Aktivitätsscore

Signifikante Unterschiede aus den Differenzen des Tegner-Aktivitätsscores für die beiden Patientenkollektive konnten nicht ermittelt werden $(p=0,537)$. 


\subsubsection{WOMAC-Score}

Im WOMAC-Score erzielten die Patienten ohne Nekrose im Mittel einen Wert von 31,11 (+/39,62) Punkten. Ihre Werte umfassten einen Bereich von minimal null und maximal 172 Punkten.

Die Patienten der Gruppe 2 lagen mit ihrem durchschnittlichen Wert bei 49,17 (+/- 57,11) Punkten. Ihre Angaben setzten sich aus Werte von minimal null und maximal 135 Punkten zusammen.

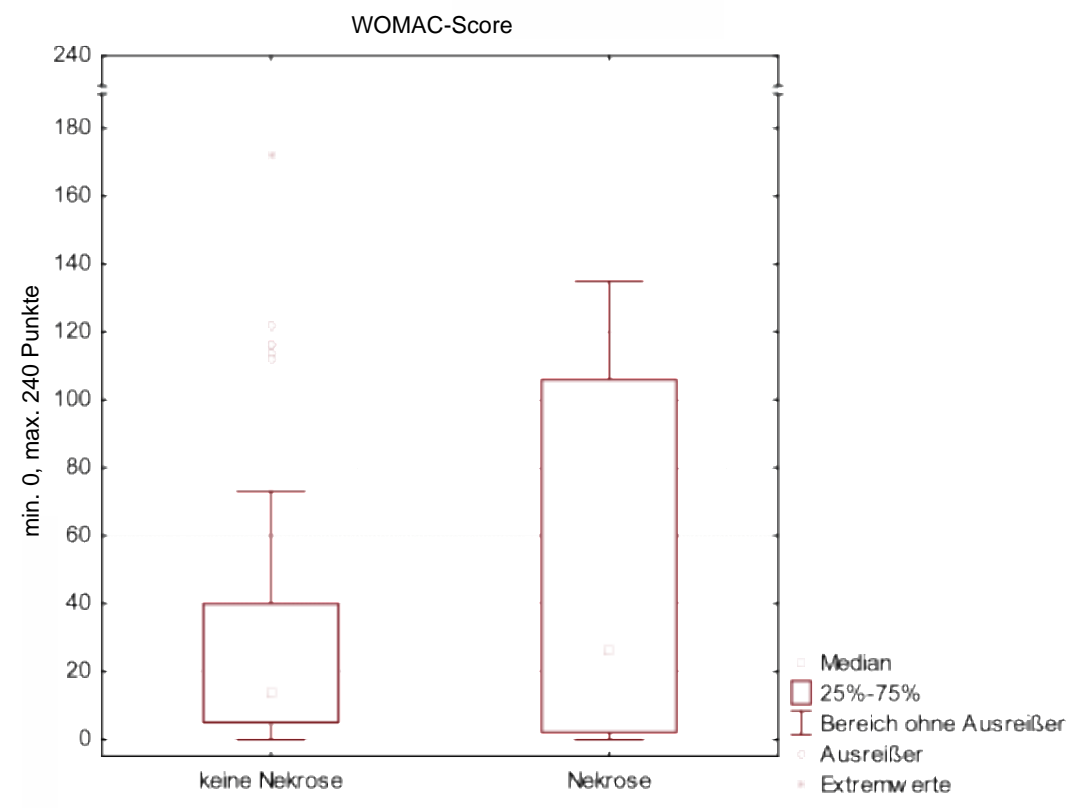

Abbildung 32: Darstellung der Ergebnisse von Patienten ohne Nekrose gegenüber Patienten mit Nekrose für den WOMAC-Score

\begin{tabular}{|c|c|c|}
\hline & Keine Nekrose & Nekrose \\
\hline Mittelwert & 31,11 & 49,17 \\
\hline Standardabweichung & 39,62 & 57,11 \\
\hline
\end{tabular}

Tabelle 35: Überblick über die Ergebnisse des WOMAC-Scores

Im WOMAC-Score konnten zwischen beiden Gruppen kein signifikanter Unterschied festgestellt werden $(p=0,562)$. 


\subsubsection{WOMAC-Score-Gesamtindex}

Im WOMAC-Score-Gesamtindex belief sich der durchschnittliche Wert der Patienten der Gruppe 1 auf 1,22 (+/- 1,52) Punkte. Dies ergab sich aus minimalen Werten von null bis zu 6,78 Punkten in der Gruppe 1.

Bei einem Wert von 2,03 (+/- 2,25) Punkten im Mittel lagen die Resultate der Gruppe 2 etwas höher. Dies resultierte aus Werten zwischen null und 5,02 Punkten der Patienten.

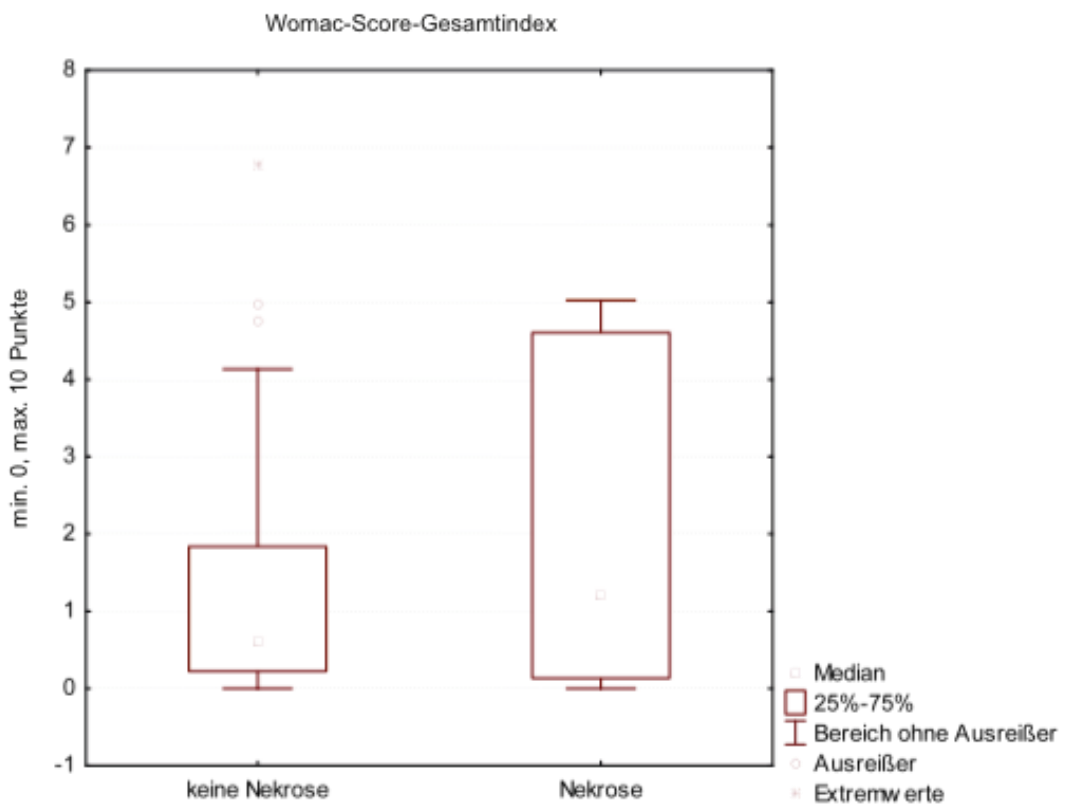

Abbildung 33: Darstellung der Ergebnisse von Patienten ohne Nekrose gegenüber Patienten mit Nekrose für den WOMAC-Score-Gesamtindex

\begin{tabular}{|c|c|c|}
\hline & Keine Nekrose & Nekrose \\
\hline Mittelwert & 1,22 & 2,03 \\
\hline Standardabweichung & 1,52 & 2,25 \\
\hline
\end{tabular}

Tabelle 36: Überblick über die Ergebnisse des WOMAC-Score-Gesamtindexes

Die Ergebnisseim WOMAC-Score-Gesamtindex für die zwei Patientenkollektive waren nicht signifikant $(p=0,622)$. 


\subsubsection{Lysholm-Score}

Im Lysholm-Score konnten für die Patienten ohne Nekrosen (Gruppe 1) ein Wert von durchschnittlich 80,37 (+/- 17,51) Punkten ermittelt werden. Die Ergebnisse der Gruppe 2 ergaben im Mittel einen Wert von 71,5 (+/- 26,41) Punkten.

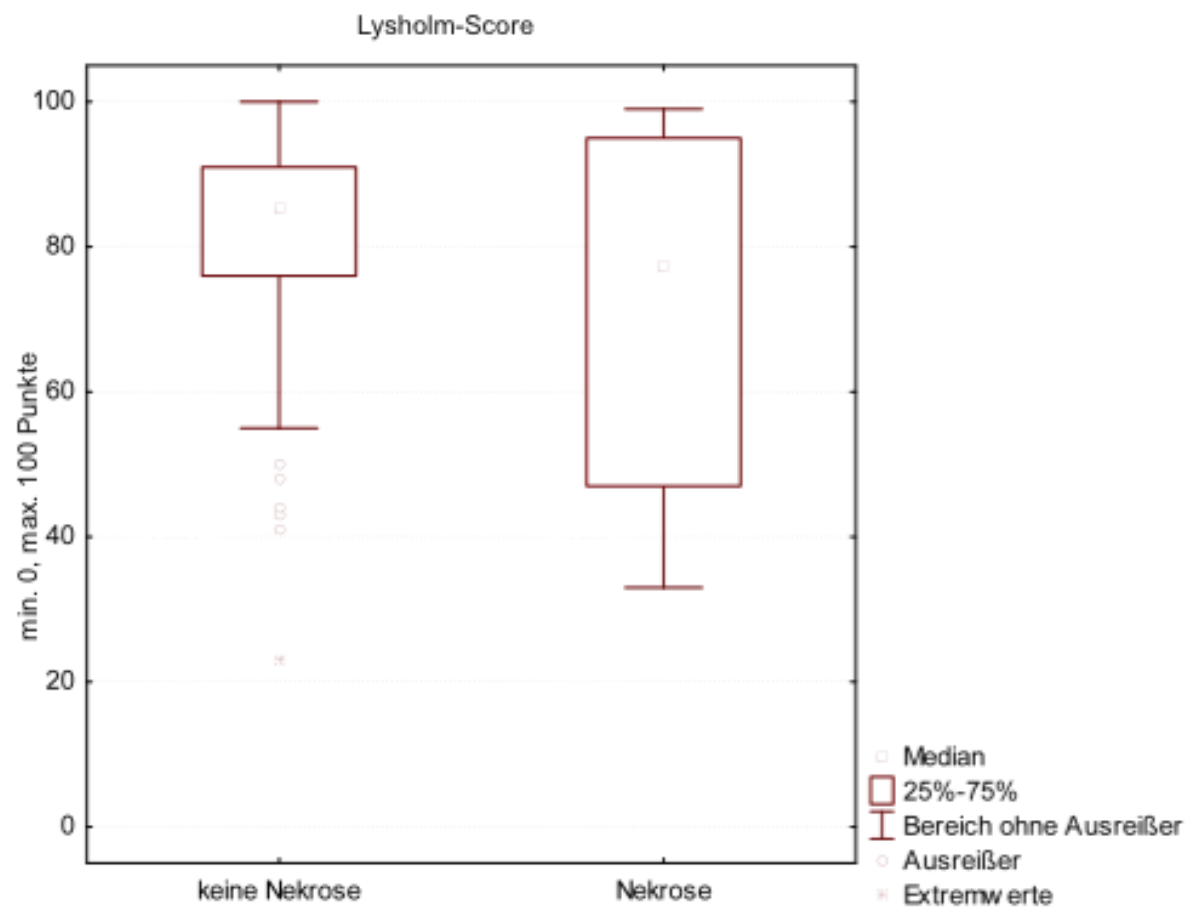

Abbildung 34: Darstellung der Ergebnisse von Patienten ohne Nekrose gegenüber Patienten mit Nekrose für den Lysholm-Score

\begin{tabular}{|c|c|c|}
\hline & Keine Nekrose & Nekrose \\
\hline Mittelwert & 80,37 & 71,5 \\
\hline Standardabweichung & 17,51 & 26,41 \\
\hline
\end{tabular}

Tabelle 37: Überblick über die Ergebnisse des Lysholm-Scores

Signifikante Unterschiede im Lysholm-Score zwischen den beiden Patientenkollektiven konnten nicht festgestellt werden $(p=0,444)$. 


\subsubsection{Patientenzufriedenheit}

Die Patienten der Gruppe 1 gaben im Mittel einen Wert von 8,43 (+/- 2,20) Punkten auf der visuell numerischen Analogskala Schmerz an.

Ein leicht niedrigerer Wert wurde für die Patienten der Gruppe 2 ermittelt. Ihr Wert lag im Durchschnitt bei 8,17 (+/- 2,48) Punkten.

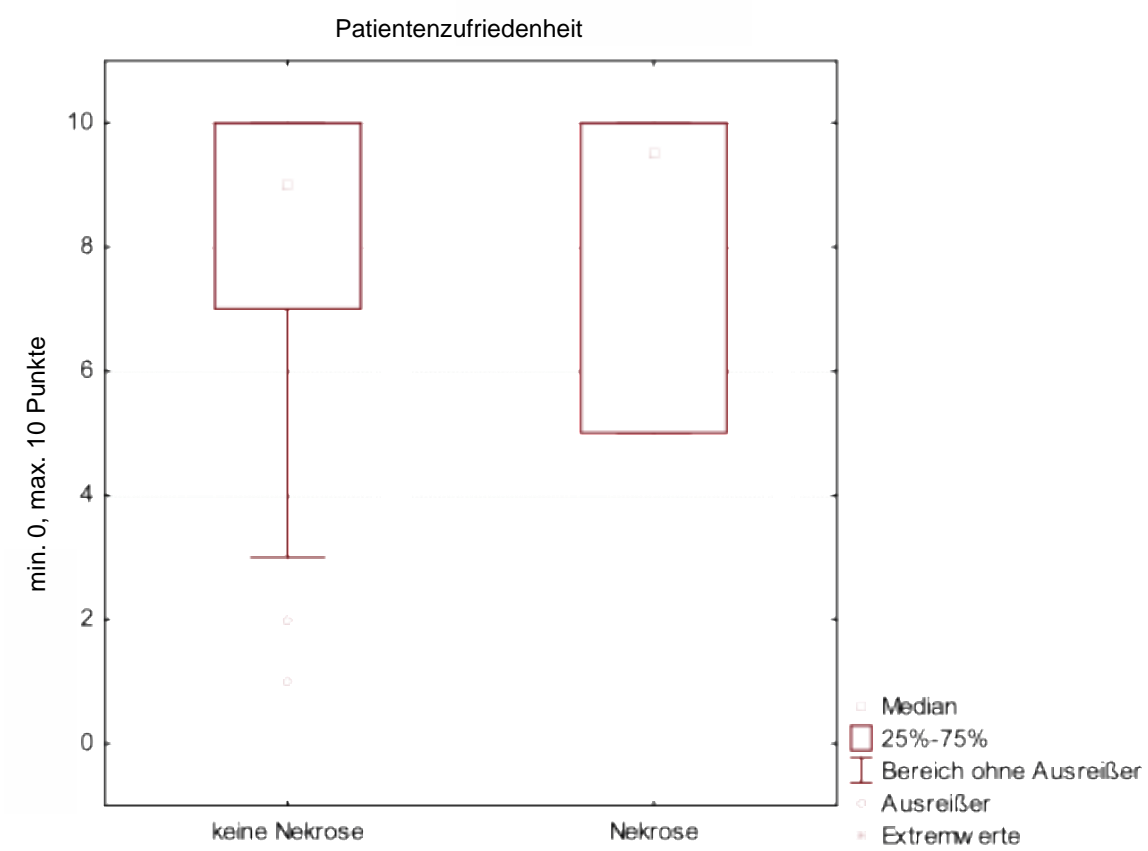

Abbildung 35: Darstellung der Ergebnisse von Patienten ohne Nekrose gegenüber Patienten mit Nekrose für die subjektive Patientenzufriedenheit

\begin{tabular}{|c|c|c|}
\hline & Keine Nekrose & Nekrose \\
\hline Mittelwert & 8,43 & 8,17 \\
\hline Standardabweichung & 2,20 & 2,48 \\
\hline
\end{tabular}

Tabelle 38: Überblick über die Ergebnisse der Patientenzufriedenheit

Die Unterschiede in der Zufriedenheit der zwei Patientenkollektive waren nicht signifikant zueinander $(p=0,911)$. 


\subsection{Histologische Untersuchung der Patella}

In der histologischen Untersuchung wurden jeweils aus dem distalen und proximalen Anteil der Patella Proben entnommen und analysiert.

Hierbei konnten in dem Abschnitt ohne radiologische Auffälligkeiten (distaler Pol) keine pathologischen Veränderungen an den Osteozyten festgestellt werden.
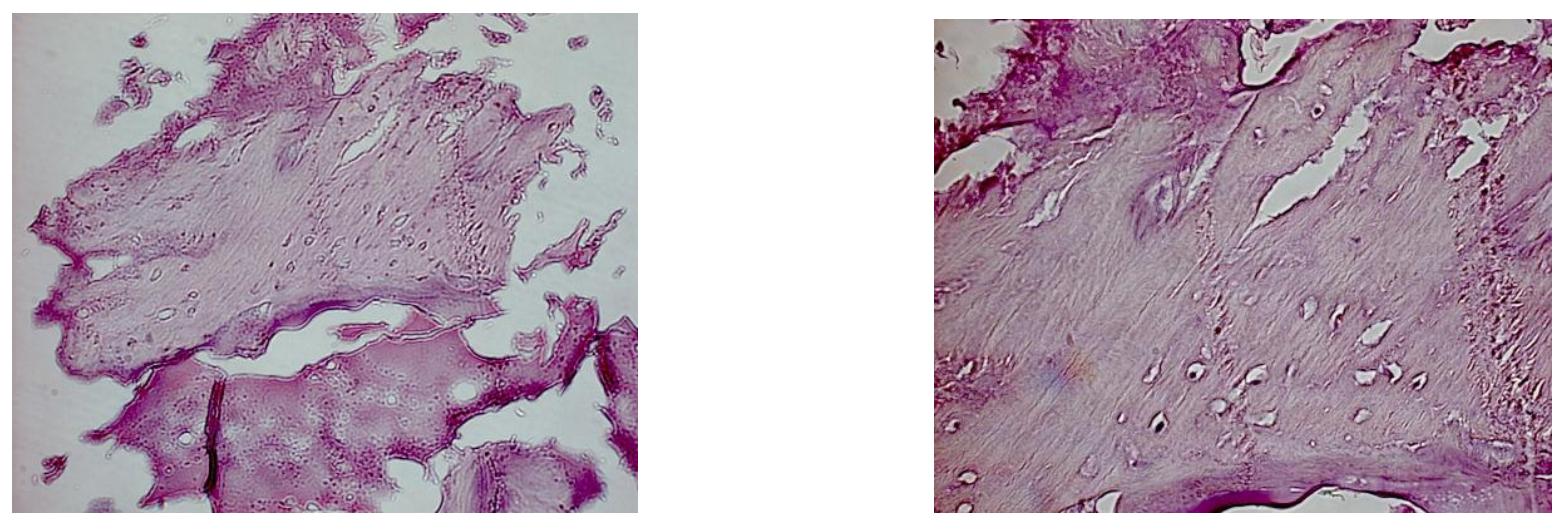

Abb. 36, 37: Histologien aus dem distalen Pol mit vitalen Osteozytenhöhlen eines Patienten (20- und 40fache Vergrößerung)

Dementgegen konnte aufgezeigt werden, dass sich in dem radiologisch nachgewiesenen hyperdensen Areal (proximaler Pol) avitale Osteozytenhöhlen befinden. Dies entspricht nekrotischem Knochen.
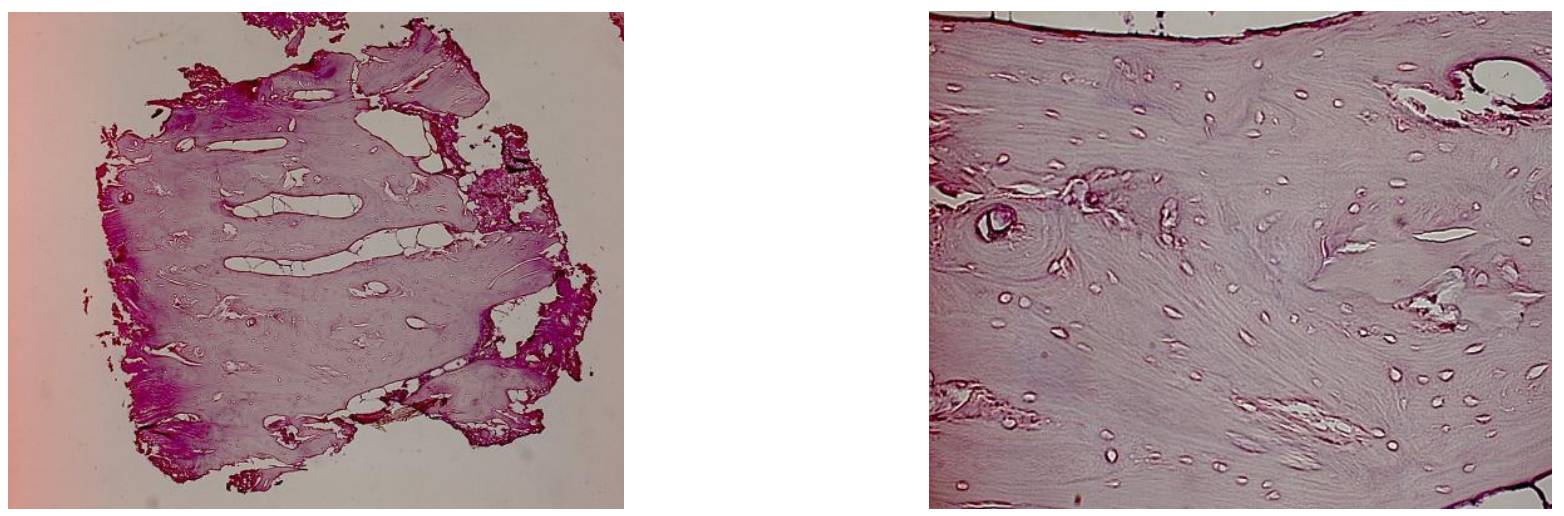

Abb. 38, 39: Histologien aus dem proximalem Pol mit avitalen Osteozytenhöhlen eines Patienten (20- und 40fache Vergrößerung) 


\section{Diskussion}

Die osteosynthetische Versorgung einer Patellafraktur dient der möglichst genauen Adaptation der Fragmente, um optimale Bewegungsvorgänge im Femoropatellargelenk wiederherzustellen und die Risiken für eventuelle postoperative Komplikationen zu minimieren.

Wie das Outcome der Patienten nach einer erlittenen Fraktur der Patella aussieht und ob bestimmte Faktoren einen Einfluss auf das Ergebnis und z.B. die subjektive Zufriedenheit der operativ versorgten Patienten nehmen, wurde in der vorliegenden Arbeit analysiert. Außerdem sollte insbesondere untersucht werden, welchen Einfluss radiologisch erfasste hyperdense Areale auf das klinische Ergebnis der Patienten besitzen.

Es konnten bei den im Rahmen der klinischen Nachuntersuchung durchgeführten Analysen aller Patienten keine signifikanten Unterschiede zwischen Längs-, Quer- oder Trümmerfrakturen festgestellt werden. In allen untersuchten Scores konnte zwischen den drei Frakturtypen keine relevanten Unterschiede festgestellt werden.

Außerdem wurde analysiert, ob zwischen Patienten, die in den postoperativen Röntgenaufnahmen hyperdense Areale in der Patella aufwiesen und Patienten, die keine radiologischen Besonderheiten besaßen, Unterschiede in der klinischen Nachuntersuchung bestehen.

Es konnte zwischen diesen beiden Patientenkollektiven in allen durchgeführten Scores kein signifikanter Unterschied festgestellt werden. Allerdings sei angemerkt, dass die Ergebnisse der Patienten mit einem postoperativ festgestellten hyperdensen Areal tendenziell schlechter ausfielen.

Des Weiteren wurden zwei Patientenkollektive gebildet, die sich durch das Vorhandensein von radiologisch-sichtbaren postoperativen Komplikationen (z.B. eine Stufe) unterschieden haben. Bei diesen beiden Patientenkollektiven konnte kein signifikanter Unterschied zwischen Patienten, die z.B. postoperativ eine Stufe in der retropatellaren Gelenkfläche aufwiesen und Patienten, ohne verbliebene Auffälligkeiten nach operativer Versorgung beobachtet werden.

Bisherige Analysen von Patellafrakturen sind meistens nur bedingt zufriedenstellend in Bezug auf tendenziell geringe Fallzahlen und weitestgehend inkonsistente Patientenkollektive, die sich in z.B. Art der Fraktur oder Verletzungsmechanismus deutlich voneinander unterscheiden (Wendl et al. 2002). 
Prinzipiell ist in durchgeführten Studien zu Patellafrakturen zu beobachten, dass die klinischen Ergebnisse zu einem überwiegenden Teil in guten bis sehr guten Bereichen angesiedelt sind (Levack et al. 1985, Wild et al. 2008).

Allerdings wird auch darauf hingewiesen, dass bei vielen Studien Frühuntersuchungen als Ergebnisse für Analysen dienten. Somit könnten eventuelle Spätfolgen der Frakturen wie z.B. retropatellare Arthrosen unter Umständen nicht berücksichtigt und folglich sollten die mehrheitlich positiven Ergebnisse eher zurückhaltend beurteilt werden.

Labitzke und Rehn (1973) konnten bei postoperativen Nachuntersuchungen feststellen, dass von den mit einer Zuggurtung operativ versorgten Patellafrakturen bei 73 \% der Patienten ein gutes bis sehr gutes Ergebnis erzielt wurde.

Levack et al. (1985) zeigten auf, dass unter den 30 mit einer Osteosynthese versorgten Patienten 19 (64\%) ein sehr gutes bis mindestens zufriedenstellendes Ergebnis erzielten und ca. ein Drittel der Patienten (36\%) ein insgesamt schlechtes Resultat aufwies.

Sie konnten weiterhin feststellen, dass unter den 30 versorgten Patienten, 16 Patienten eine Zuggurtung erhielten und 14 Patienten mit einer reinen Drahtcerclage um die Patella versorgt wurden. Nur zwei der mit einer Zuggurtung versorgten Patienten (14\%) wiesen ein schlechtes Resultat auf, wohingegen insgesamt neun der 16 Patienten (52\%) mit einer Cerclage ein schlechtes Ergebnis erzielten.

Sie schlossen sich daraufhin der ebenso von anderen Studien (Böstman et al. 1983) aufgestellten These an, dass die Versorgung einer Querfraktur der Patella mit einer Zuggurtung das beste Outcome für den Patienten aufweist, da die Patienten früh mit ihrer Nachbehandlung beginnen können und ihre Zeit bis zur Regeneration möglichst gering gehalten werden kann.

Böstman et al. (1983) stellten in ihrer Studie fest, dass 71 von 93 Patienten (76\%) ein exzellentes oder gutes Ergebnis erzielten. Des Weiteren konnten bei Patienten die jünger als 30 Jahren waren tendenziell bessere Werte erzielt werden als bei älteren Patienten und die Ergebnisse bei einfachen Querfrakturen waren ebenso insgesamt besser als diejenigen von Patienten mit Trümmerfrakturen.

Wild et al. (2008) untersuchten die postoperativen Ergebnisse bei 50 Patienten, deren Frakturen mit einer Zuggurtung versorgt wurden. Sie stellten bei $70 \%$ der Patienten ein sehr gutes bis gutes Ergebnis und nur bei $10 \%$ ein schlechtes Ergebnis fest, obwohl sie bei 40 der 50 Patienten (80\%) Minor- (Implantatkomplikationen und Gelenkstufen $\leq 2 \mathrm{~mm}$ ) oder 
Majorkomplikationen (Infektionen und Frakturdehiszenzen bzw. Gelenkstufen $>2 \mathrm{~mm}$ ) in den postoperativen radiologischen Aufnahmen fanden.

Zwischen den radiologischen Aufnahmen und den klinischen Ergebnissen konnte kein statistischer Zusammenhang festgestellt werden.

Jaskulka et al. (1989) untersuchten in ihrer Analyse 48 operativ versorgte Patellafrakturen. 82,2 \% ihrer Patienten erzielten ein sehr gutes bis zufriedenstellendes Ergebnis, die restlichen 18,8 \% wurden als schlecht eingestuft. Die Nachuntersuchung erfolgte zwischen drei und zehn Jahren nach der operativen Versorgung und wurde über ein eigenes Punktesystem ausgewertet.

Die Autoren schlossen sich der Meinung an, dass eine reine Versorgung mittels einer Cerclage nicht angestrebt werden sollte (Levack et al., 1985), da eine übungsstabile Nachbehandlung nicht möglich ist und eine über Wochen zu tragende Gipsschiene gegenüber einer Versorgung mittels einer Zuggurtungsosteosynthese, die frühere Belastungen ermöglicht, deutlich mehr Nachteile bietet und schlechtere Ergebnisse resultieren.

Bei Längs- und isolierter Querfraktur kann eine Versorgung mit Zugschrauben erwogen werden, aber prinzipiell für alle Frakturformen empfohlen Jaskulka et al. (1989) die am meisten verbreitete Versorgung über die Zuggurtungsosteosynthese.

Sie hielten weiterhin fest, dass man bei komplizierten Frakturen nicht definitiv alle Fragmente adaptieren muss, sondern durchaus eine Teilresektion in Betracht zu ziehen sei. So konnten Jaskulka et al. (1989) in ihrer Studie gute Resultate nach Teilresektionen feststellen und eventuelle postoperative Komplikation wie Teilarthrosen würden durch unnötige Adaptierung verhindert.

Tendenziell ist in der von uns durchgeführten Studie zu beobachten, dass die Patienten mit einer Längsfraktur die besten postoperativen Ergebnisse erzielten.

Das Ergebnis war jedoch nicht signifikant gegenüber den anderen beiden Frakturtypen (Quer- und Trümmerfraktur).

So wiesen die Patienten mit einer Längsfraktur im Vergleich zu Patienten mit einer Queroder Trümmerfraktur beispielsweise einen durchschnittlich geringeren Wert in der visuell numerischen Analogskala Schmerz auf und hatten eine geringere Differenz im TegnerAktivitätsscore (geringere Aktivitätseinbuße prä- zu postoperativ) als die anderen beiden Patientenkollektive.

Diese Tendenz kann weiterhin auch bei den anderen verwendeten Scores beobachtet werden. 
Diese Ergebnisse bekräftigen die in der Einleitung genannte These und stehen in Einklang mit den Überlegungen Scapinellis (1967), dass Patienten mit einer Längsfraktur aufgrund der anatomischen Verhältnisse der Patella bessere Heilungschancen besitzen.

Denn gegenüber Quer- oder Trümmerfrakturen entstehen keine minderperfundierten Areale und eine schnellstmögliche Heilung und Adaptierung der Fragmente kann ohne postoperative Heilungsstörungen gelingen.

Weiterhin konnten wir feststellen, dass die Ergebnisse der Patienten mit Querfrakturen in allen Scores schlechter ausfielen als diejenigen der Patienten mit Trümmerfrakturen.

Insgesamt konnte bei allen drei Frakturtypen eine subjektive Patientenzufriedenheit von durchschnittlich mehr als acht der maximal möglichen zehn Punkte notiert (gutes bis exzellentes Ergebnis) werden. Diese Werte zeigen ähnliche Resultate wie in bisher durchgeführten Studien (Labitzke und Rehn 1973, Pankarter et al. 1979, Wild et al. 2010). Tendenziell kann man also davon ausgehen, dass Patienten mit einer Patellafraktur in der Vielzahl der Fälle mit ihrem postoperativen Ergebnis zufrieden sind.

Wie bereits in der Einleitung und in der Studie von Scapinelli (1967) übereinstimmend beschrieben, treten im Rahmen von postoperativen Komplikationen Nekrosen in der Patella primär bei Quer- aber auch bei Trümmerfrakturen auf.

In unserer durchgeführten Studie konnten bei sechs Patienten in den postoperativen radiologischen Aufnahmen hyperdense Bereiche entdeckt werden, weshalb diese anschließend mit den anderen Patienten verglichen wurden.

In den analysierten Scores konnte prinzipiell festgestellt werden, dass die Patienten ohne hyperdense Areale der Patella bessere Resultate gegenüber den Patienten mit hyperdensen Arealen erzielten.

So waren z.B. in der visuell numerischen Analogskala Schmerz die Ergebnisse der Patienten mit hyperdensen Arealen durchschnittlich einen Punkt höher als in der Vergleichsgruppe. Diese Unterschiede spiegelten sich darüber hinaus in den anderen verwendeten Scores wider:

Die Differenz im Tegner-Aktivitätsscore postoperativ betrug für Patienten ohne hyperdense Areale $0,7(+/-1,0)$ Punkte und für Patienten mit hyperdensen Arealen 1,0 (+/- 1,26) Punkte. Zwischen den beiden Patientenkollektiven konnten für die Scores keine signifikanten Unterschiede festgestellt werden, allerdings stellen die Ergebnisse konsequente Resultate dar, die jeweils schlechtere Ergebnisse für die Patienten mit hyperdensen Arealen aufwiesen. 
Die Ergebnisse bekräftigen die Annahme, dass die ossäre Heilung der Patella bei Quer- und Trümmerfrakturen eingeschränkt bzw. verzögert sein kann.

Unter den Patienten mit postoperativ nachgewiesenen hyperdensen Arealen der Patella waren in dieser Studie jeweils drei Patienten mit einer Quer- und Trümmerfraktur. Dementgegen wurde diese postoperative Komplikation nicht bei Patienten mit Längsfrakturen beobachtet, weil die Fragmente einer Längsfraktur postoperativ weiterhin suffizient mit Blut versorgt werden und die distrahierenden Kräfte des Musculus quadriceps femoris bei dieser Frakturform, im Gegensatz zu einer Querfraktur, nicht von relevanter Bedeutung sind (Scapinelli 1967).

Scapinelli (1967) konnte unter 162 untersuchten Patienten mit einer Querfraktur 41 (25,3\%) Patienten entdecken, die osteonekrotische Anteile in der Patella aufwiesen. Es sei darauf hingewiesen, dass es sich bei den meisten dieser Frakturen um dislozierte Querfrakturen mit einem deutlich dehiszenten Frakturspalt handelte, wodurch eine arterielle Perfusion der proximalen Patellaanteile nicht mehr gewährleistet war.

Ein maximaler Unterschied in der Densität der beiden Fragmente konnte ein bis zwei Monate nach der Operation festgestellt werden, welcher anschließend kontinuierlich abnahm. Nach ca. sechs Monaten konnten in der Densität der Frakturfragmente keine Unterschiede mehr feststellt werden.

Sechs bis acht Monate nach der Operation stellte Scapinelli im Rahmen von klinischen Untersuchungen fest, dass die Patienten mit Osteonekrosen gegenüber den anderen Patienten keine relevanten Unterschiede aufwiesen und ähnliche postoperative Ergebnisse erreicht wurden.

Die Autoren empfahlen darüber hinaus, dass bei einer Entscheidung zur partiellen Patellektomie der proximale Anteil einer Querfraktur favorisiert werden könne, da nur in diesem Anteil Osteonekrosen zu erwarten seien. Der distale Anteil sollte, wenn möglich, aufgrund seiner doppelten Blutversorgung und folglich besseren Heilungschance belassen werden.

Diese Ergebnisse können im Einklang mit den in unserer Studie ermittelten Daten gesehen werden. So waren zwischen den beiden Patientenkollektiven postoperativ keine signifikanten Unterschiede auszumachen und nach abgeschlossener Konsolidierung der Frakturfragmente erzielten die Patienten ähnliche Resultate in den analysierten Scores.

Die osteonekrotischen Anteile stellen eine mögliche postoperative Erscheinung dar, die bei Quer- und Trümmerfrakturen auftreten kann, allerdings resultieren auf langfristige Sicht keine definitiven Effekte für den Patienten. 
Bei den Patienten mit einem osteonekrotischen Anteil wurde eine mittlere Zeit zwischen Fraktur und operativer Versorgung von 124 (+/-201;3-525) Stunden festgestellt. Bei den Patienten ohne diese Heilungsstörung lag die Zeit bei 92,1 (+/-104,4;1-504) Stunden. Allerdings tritt diese Variable im Vergleich zu der Frakturform in den Hintergrund, da allein die Frakturform und nicht die Zeit bis zur operativen Versorgung als determinierend für das Auftreten eines hyperdensen Areals erscheint.

Es sollte jedoch darauf verwiesen werden, dass es sich bei der von uns durchgeführten Studie um ein relativ kleines Patientenkollektiv handelt.

Unabhängig davon wurden noch die Patienten mit radiologischen Auffälligkeiten (Stufen) im Vergleich zu den restlichen Patienten untersucht.

Dick et al. (1975) zeigten in ihrer Studie, wie wichtig eine stufenlose Reposition der Frakturfragmente ist. Von 23 Patienten, die postoperativ eine Stufe aufwiesen, waren nur drei Patienten $(=13 \%)$ beschwerdefrei und gleichzeitig besaßen diese Patienten als Spätfolge der Patellafraktur ein erhöhtes Risiko für die Entwicklung einer frühzeitig einsetzenden Arthrose.

Der einseitig auftretende Knorpelschaden (Chondromalazia patellae) kann entweder allein aus der direkten Krafteinwirkung während des Traumas oder durch die Fraktur selber entstehen (Dick et al. 1975, Wirth et al. 2005).

Zwar können diese Beschwerden auch bei absolut stufenfreier Adaptation der Fragmente auftreten, allerdings konnte gezeigt werden, dass Patienten z.B. mit komplizierten Trümmerfrakturen bei stufenloser Adaptation völlig beschwerdefrei sein können (Dick et al. 1975).

Labitzke und Rehn (1973) wiesen darauf hin, dass das Risiko einer möglichen Femoropatellararthrose bei einer Teilresektion oder aufgrund einer verbliebenen Stufe von der erhaltenen Fläche der Patella abhängt. Da die Patella mit der gesamten Rückseite artikuliert sind zufriedenstellende Ergebnisse wahrscheinlicher, wenn man nur maximal ein Drittel der Patella reseziert, was auch von anderen Studien unterstützt wurde (Tscherne und Szyszkowitz 1969).

Ähnliche Resultate zeigten Dietz et al. (2009) auf und stellten die Indikation zu einer Revisionsoperation bei verbliebener postoperativer Stufe von mehr als 2 Millimetern, da eine frühzeitig einsetzende Arthrose im Femoropatellargelenk unausweichlich eintrete. 
Sie formulierten diese These aufgrund der Ergebnisse von Böstman et al. (1981), die im Rahmen einer Studie bei klinischen Nachuntersuchungen keine relevanten Unterschiede bei Patienten feststellen konnten, die eine verbliebene Stufe von maximal zwei Millimetern aufwiesen. Wohingegen bei zwei Patienten, die eine Stufe von mehr als zwei Millimetern aufwiesen, nur nicht zufriedenstellende Ergebnisse beobachtet werden konnten.

Unter den Patienten in unserer Studie mit einer verbliebenen Stufe fanden sich sechs Patienten mit einer Quer- und sieben Patienten mit einer Trümmerfraktur. Drei Patienten wiesen eine Stufe kleiner als einen Millimeter auf, neun Patienten hatten eine Stufe zwischen einem und drei Millimeter und ein Patient wies eine Stufe größer als drei Millimeter auf.

Es konnten insgesamt keine signifikanten Unterschiede zu den anderen Studienteilnehmern festgestellt werden. Allerdings konnte mit einer Differenz im Tegner-Aktivitätsscore von 0,92 $(+/-0,95)$ Punkten der höchste Wert für ein Patientenkollektiv in dieser Studie festgestellt werden (Funktionseinbuße von prä- zu postoperativ).

Auffällig war das nachgewiesene nicht zufriedenstellende Ergebnis bei dem Patienten mit mehr als drei Millimetern Stufe. Der Patient gab in der klinischen Nachuntersuchung für die visuell numerische Analogskala Schmerz mit fünf Punkten den höchsten Wert an und verschlechterte sich im Tegner-Aktivitätsscore von präoperativen sieben auf postoperative vier Punkte, wodurch er mit einer Differenz von drei Punkten deutlich über dem Durchschnitt lag.

Die Ergebnisse dieses Patienten können in Zusammenhang mit den bisher durchgeführten Studien gesehen werden.

Die Problematik erscheint bei größeren Stufen relevanter zu werden und das Risiko für langfristige postoperative Komplikationen wie z.B. posttraumatische Arthrosen steigt (Carpenter et al. 1993, Wild et al. 2008).

Die Ergebnisse unserer Studie müssen zur weiteren Verifizierung im Rahmen von zukünftigen Studien mit größeren Fallzahlen und z.B. randomisierten Patienten untersucht werden. Dabei könnte die Aussagekraft der Studie z.B. durch standardmäßig durchgeführte computertomographische Aufnahmen und histologische Untersuchungen erhöht werden. 


\section{Schlussfolgerungen}

Im Rahmen der klinischen Untersuchung und der dabei durchgeführten Analyse relevanter Scores konnten keine signifikanten Unterschiede zwischen den einzelnen Frakturtypen beobachtet werden.

Bei den Patienten mit postoperativ nachweisbaren osteonekrotischen Arealen konnten tendenziell schlechtere klinische Ergebnisse nachgewiesen werden. Bei der kleinen Fallzahl der Patienten mit dieser Komplikation konnte jedoch keine Signifikanz festgestellt werden.

Die Zeit zwischen Trauma und operativer Versorgung scheint in der Bedeutung auf das mögliche Entstehen einer Osteonekrose leichten Einfluss zu haben. Die Gruppe mit Osteonekrosen wurde tendenziell später operiert als diejenige ohne Nekrosebildung. Eine frühzeitige Versorgung von Patellafrakturen sollte deshalb angestrebt werden.

Es konnte gezeigt werden, dass sich das klinische Ergebnis bei einer Stufe von mehr als drei Millimetern als nicht zufriedenstellend herausstellt und deshalb eine frühzeitige operative Revision in Erwägung gezogen werden sollte.

\section{Zusammenfassung}

Patellafrakturen können auch bei optimaler operativer Versorgung zu erheblichen Problemen für den Patienten führen. Insbesondere Materialdislokationen, Heilungsstörungen und Pseudarthrosen stellen die überwiegenden Komplikationen nach Patellafraktur dar. Ziel dieser klinischen, retrospektiven Studie war es, das klinische und radiologische Ergebnis operativ versorgter Patellafrakturen zu evaluieren.

Es sollte insbesondere untersucht werden, welchen Einfluss radiologisch erfasste hyperdense Areale auf das klinische Resultat haben.

Anhand von standardisierten Nachuntersuchungsbögen (American Knee Society Score, Tegner-Aktivitätsscore, Lysholm-Score, Western Ontario and McMaster Universities-Score, visuell numerische Analogskala Schmerz, subjektive Patientenzufriedenheit) wurden die Patienten klinisch nachuntersucht und deren Anamnese erfasst. Ergänzend wurden die radiologischen Aufnahmen nach einem selbst erstellten Bewertungsschema beurteilt (Frakturtyp, Stufen, Defekte, hyperdense Areale) und ein Vergleich zu den klinischen Ergebnissen der Patienten ohne radiologische Besonderheiten gezogen. Von der Studie 
ausgeschlossen wurden Patienten, die eine beidseitige Fraktur erlitten haben, nicht mittels einer Osteosynthese versorgt (Patellektomie) oder konservativ therapiert wurden.

Insgesamt konnten 60 Patienten in die Studie eingeschlossen werden, deren Patellafrakturen über eine Schraubenosteosynthese, eine Zuggurtungsosteosynthese oder eine Kombination von Osteosynthesematerialien operativ versorgt wurden,

Die Patienten fanden sich im Mittel nach 60,6 (+/- 33,88) Monaten zur klinischen Nachuntersuchung ein.

Es zeigte sich bei den Ergebnissen zwischen den drei Frakturtypen kein signifikanter Unterschied in den verwendeten klinischen Scores. Die Patienten mit einer Längsfraktur erreichten im AKSS eine durchschnittliche Punktzahl von 177 (+/- 15,55), im TegnerAktivitätsscore präoperativ einen Wert von 4,57 (+/- 1,99) und postoperativ von 4,43 (+/2,07). Im WOMAC-Score wurden für die Gruppe im Mittel 18,43 (+/- 23,55) Punkte notiert, wodurch sich ein WOMAC-Score-Gesamtindex von 0,73 (+/- 1,15) ergab. Im Lysholm-Score erreichten die Patienten im Mittel 86,86 (+/- 10,68) Punkte.

Patienten mit einer Querfraktur erzielten im AKSS eine durchschnittliche Punktzahl von 160 (+/- 35,94), im Tegner-Aktivitätsscore präoperativ einen Wert von 4,93 (+/- 1,84) und postoperativ von 4,07 (+/- 2,12). Im WOMAC-Score wurden für die Querfrakturen im Mittel 41,86 (+/- 50,99) Punkte notiert, wodurch ein WOMAC-Score-Gesamtindex von 1,64 (+/1,96 ) errechnet wurde und im Lysholm-Score erzielten die Patienten mit einer Querfraktur durchschnittlich 74,75 (+/- 20,19) Punkte.

Für die Patienten mit einer Trümmerfraktur wurde im AKSS eine durchschnittliche Punktzahl von 171,88 (+/- 25,91) erzielt, im Tegner-Aktivitätsscore präoperativ ein Wert von 5,28 (+/1,65) und postoperativ von 4,52 (+/- 1,81) Punkten ermittelt. Für die Trümmerfrakturen wurden im WOMAC-Score im Mittel 26,96 (+/- 31,02) Punkte notiert, wodurch ein WOMACScore-Gesamtindex von 1,09 (+/- 1,17) erzielt wurde und im Lysholm-Score konnten durchschnittlich $82,72(+/-17,33)$ Punkte erreicht werden.

Von den untersuchten Teilnehmern wiesen 6 von 60 Patienten radiologisch ein hyperdenses Areal in ihrer Patella auf. Zwischen den Ergebnissen der Patienten mit Auffälligkeiten in den postoperativen Aufnahmen und den Patienten ohne diese nachgewiesenen Areale zeigten sich keine signifikanten Unterschiede. Patienten ohne hyperdense Areale erzielten im AKSS eine durchschnittliche Punktzahl von 167,5 (+/- 30,28), im Tegner-Aktivitätsscore präoperativ einen Wert von 5,04 (+/- 1,83) und postoperativ von 4,33 (+/- 2,06). Im WOMAC-Score wurden für die Patienten 31,11 (+/- 39,62) Punkte notiert, wodurch ein WOMAC-Score- 
Gesamtindex von 1,22 (+/- 1, 52) Punkten errechnet wurde. Im Lysholm-Score erzielten die Patienten ohne hyperdense Areale durchschnittlich 80,37 (+/- 17,51) Punkte.

Im Vergleich dazu erzielten die Patienten mit radiologisch hyperdensen Arealen im AKSS eine durchschnittliche Punktzahl von 161,83 (+/- 35,58), im Tegner-Aktivitätsscore präoperativ einen Wert von 5,00 (+/- 1,09) und postoperativ von 4,00 (+/- 0,89) Punkten. Im WOMAC-Score wurden für die Gruppe im Mittel 49,17 (+/- 57,11) Punkte notiert, wodurch sich ein WOMAC-Score-Gesamtindex von 2,03 (+/- 2,25) ergab. Im Lysholm-Score erreichten die Patienten mit hyperdensen Arealen im Mittel 71,5 (+/- 26,41) Punkte.

Anhand von histologischen Untersuchungen konnte bestätigt werden, dass die vorliegenden hyperdensen Areale partielle Osteonekrosen darstellen.

Schlussfolgernd sind radiologisch hyperdense Bereiche nach Patellafraktur als eine postoperative Heilungsstörung anzusehen. Langfristig scheinen diese keinen definitiven Einfluss auf das klinische Resultat der Patienten zu haben. Jedoch war zu beobachten, dass die Ergebnisse der Patienten mit postoperativ hyperdensen Arealen tendenziell schlechter gegenüber denen der Patienten ohne diese Auffälligkeiten ausfielen. 


\section{Literaturverzeichnis}

Arnold, MP, Friedereich NF, Widmer H, Müller W (1998): Patellaersatz bei Knietotalendoprothesen - notwendig? Orthopäde 27, 637-641

Bellamy N, Buchanan WW (1986): A preliminary evaluation of the dimensionality and clinical importance of pain and disability in osteoarthritis of the hip and knee. Clin Rheumatol $\underline{5}, 231$ 241

Benger U, Johnell O, Redlund-Johnell I (1986): Increasing incidence of tibia condyle and patella fractures. Acta Orthop Scand $\underline{57}, 334-336$

Böhler L (1961): Behandlung der Kniescheibenbrüche. Dtsch Med Wochenschr 모, 1209

Böstman O, Kiviluoto O, Nirhamo J (1981): Comminuted displaced fractures of the patella. Injury $\underline{13}, 196-202$

Böstman O, Kiviluoto O, Santavirta S, Nirhamo O, Wilppula E (1983): Fractures of the Patella Treated by Operation. Arch Orthop Trauma Surg $\underline{10}, 78-81$

Bohr H, Larsen EH (1965): On Necrosis of the Femoral Head After Fracture of the Neck of the Femur. J Bone Joint Surg $\underline{47 \text { B }, 330-338}$

Boström A (1972): Fracture of the patella. A study of 422 patellar fractures.

Acta Orthop Scand Suppl $\underline{143}, 1-80$

Brill W, Hopf Th (1987) Biomechanische Untersuchung verschiedener Osteosyntheseverfahren bei Patella-Querfrakturen. Unfallchirurg $\underline{90}, 162-172$

Buckwalter JA, Saltzman C, Brown T (2004): The impact of osteoarthritis: implications for research. Clin Orthop Rel Res $\underline{427 S}, 6-15$

Burks RT, Desio SM, Bachus KN, Tyson L, Springer K (1998) Biomechanical evaluation of lateral patellar dislocations. Am J Knee Surg 11(1), 24-31

Cameron H, Huffer B (1985): Avascular necrosis of the patella following total 
Knee replacement. Acta Orthop Belg $\underline{51}, 805-810$

Carpenter JE, Kasman R, Matthews LS (1993): Fractures of the patella. J Bone Joint Surg Am $\underline{75}, 1550-1561$

Christensen B, Jakob RP (1992): Fractures associated with patellar ligament graft in crutiate ligament surgery. J Bone Joint Surg $\mathrm{Br} \underline{74}, 617-619$

Conlan T, Garth WP, Lemons JE (1993): Evaluation of the medial soft-tissue restraints of the extensor mechanism of the knee. J Bone Joint Surg Am 75, 682-693

Debrunner A :Orthopädie, Orthopädische Chirurgie: Patientenorientierte Diagnostik und Therapie des Bewegungsapparates, 4. Auflage, Verlag Hans Huber Bern 2005

Desio SM, Burks RT, Bachus KN (1998): Soft tissue restraints to lateral patellar translation in the human knee. Am J Sports Med 26, 59-65

Dick W, Henche HR, Morscher E (1975): Der Knorpelschaden nach Patellafraktur.

Arch Orthop Unfall-Chirur $\underline{81}, 65-76$

Dietz SO, Hessmann MH, Gercek E, Rommens PM (2009): Patellafraktur. Oper Orthop Traumatol $\underline{21}, 206-220$

Durst J: Traumatologische Praxis in einem Band: Standards in Diagnostik und Therapie für alle Fachgebiete, Schattauer Verlag Stuttgart 1997, 546

Durst J, Arnold H: Chirurgie CompactLehrbuch, Schattauer Verlag Stuttgart 1994, 483

Elkayam O, Zhukovsky G, Fishel B, Yaron M (1996): Steroid-induced avascular necrosis of the patella in an ulcerative colitis patient. Clin Exp Rheumatol 14(4), $\square$ 447-448

Endler F.: Einführung in die Biomechanik und Biotechnik des Bewegungsapparates. In: Witt AN, Rettig H, Schlegeg KF, Hackenbroch M, Hupfauer W (Hrsg): Orthopädie in Praxis und Klinik. Thieme Verlag Stuttgart 1980, 301

Feller JA, Amia AA, Andrish JT, Arendt EA, Erasmus PJ, Powers CM (2007): Surgical biomechanics of the patellofemoral joint. Arthr Rel Surg 23, 542-553 
Fischer O: Kinematik organischer Gelenke. In: Die Wissenschaft - Sammlung naturwissenschaftlicher und mathematischer Monographien. Heft 18. Vieweg Verlag, Braunschweig 1907

Franssen BBGM, van Diest PJ, Schuurman AH, Kon M (2008): Drilling K-wires, what about the osteocytes? An experimental study in rabbits. Arch Orthop Trauma Surg 128(1), 83-87

Freuler F., Brunner C (1975): Die femoro-patellare Arthrose nach operierten Patellafrakturen. Z Unfallmed Berufskr 68, 76-79

Freuler F, Brunner C, Rüter A (1975): Spätresultate bei operierten Patellafrakturen. Hefte Unfallheilkunde $\underline{120}, 68-75$

Frosch KH, Floerkemeier T, Abicht C, Adam P, Dathe H, Fanghänel J, Stürmer KM, KubeinMeesenburg D, Nägerl H (2009): Eine neuartige Knieendoprothese mit physiologischer Gelenkform. Unfallchirurg $\underline{112}, 168-175$

Fukubayashi T., Kurosawa H. (1980): The contact area and pressure distribution pattern of the knee. A study of normal and osteoarthrotic knee joints Acta Orthop Scand $\underline{51(6)}$ : 871-879

Galla M, Lobenhoffer P (2005): Frakturen der Patella. Chirurg $\underline{76}$, 987-997

Goodfellow J, Hungerford DS, Zindel M (1976a): Patello-femoral joint mechanics and pathology. I. Functional anatomy of the patello-femoral joint. J Bone Joint Surg $\underline{58-B}$, 287290

Goodfellow J, Hungerford DS, Woods C (1976b): Patello-femoral joint mechanics and pathology. II. Chondromalacia patellae. J Bone Joint Surg $\underline{58-B}, 291-299$

Gosal HS, Singh P, Field RE (2001): Clinical experience of patellar fracture fixation using metal wire or non-absorbable polyester - a study of 37 cases. Injury $\underline{32}, 129-135$

Hautamaa PV, Fithian DC, Kaufmann KR, Daniel DM, Pohlmeyer AM (1998): Medial soft tissue restraints in lateral patellar instability and repair. Clin Orthop $\underline{349}, 174-182$

Hehne HJ, Schlageter MS, Hultzsch W, Rau WS (1981) Experimentelle patello-femorale Kontaktflächenmessungen - 1. Mitteilung: Neue Aspekte zur funktionellen Anatomie. Z Orthop $\underline{119}, 167-176$ 
Hehne HJ (1990): Biomechanics of the Patellofemoral Joint and its Clinical Relevance. Clin Orthop and Rel Res $\underline{258}, 73-85$

Hepp WR: Radiologie des Femoro-Patellargelenkes. Enke Stuttgart,1983

Holtby R, Grosso P (1996): Osteonecrosis and Resorption of the Patella After Total Knee Replacement - A Case Report. Clin Orthop $\underline{328}, 155-158$

Hou S, Zhang Y, Wu W (2002): Study on characteristics of fractures from road traffic accidents in 306 cases. Chin J Traumatol $\underline{5}(1), 52-54$

Insall JN, Dorr LD, Scott RD, Scott WN (1989): Rationale of the Knee Society Clinical Rating System. Clin Orthop 248, 13-4.

Jaskulka R, Ittner G, Raffezeder U (1989): Die chirurgische Versorgung dislozierter Patellafrakturen - Therapie und Ergebnisse. Unfallchirurgie 15, 253-260

Johnson EE: Fractures of the knee. In: Rockwood CA, Green DP, Bucholz RW (eds.) Fractures in adults. JB Lippincott Philadelphia 1991 1762-1777

Kapandji IA: Funktionelle Anatomie der Gelenke Schematisierte und kommentierte Zeichnungen zur menschlichen Bewegung. Thieme Verlag Stuttgart 2006

Kaufer H (1971): Mechanical Function of the Patella. J Bone Joint Surg Am $\underline{53}, 1551-1560$

Kellgren JH, Lawrence JS (1957): Radiological assessment of osteo-arthrosis. Ann Rheum Dis $\underline{16}, 494-502$

Labitzke R, Rehn J (1973): Zur Behandlung von Patellafrakturen. Arch Orthop Unfallchir $\underline{77}$, 64-74

Lennox IA, Cobb AG, Knowles J, Bentley G (1994): Knee function after patellectomy. A $12-$ to 48-year follow-up. J Bone Joint Surg Br $\underline{76-\mathrm{B}}$, 485-487

Levack B, Flannagan JP, Hobbs S (1985): Results of surgical treatment of patellar fractures. J Bone Joint Surg Br $\underline{67}, 416-419$ 
Lysholm J, Gillquist J (1982): Evaluation of knee ligament surgery results with special emphasis on use of a scoring scale. Am J Sports Med 10, 150-154

Maquet P (1977): The Biomechanics of the Knee and Surgical Possibilities of Healing Osteoarthritic Knee Joints. Clin Orthop 146, 102-110

Menschik A (1974): Mechanik des Kniegelenks. Z Orthop 112, 481-495

Müller ME, Allgöwer M, Schneider R, Willenegger H: Manual of Internal Fixation. Techniques recommended by the AO-Group Springer Verlag Berlin 1979

Nägerl H, Kubein-Meesenburg D, Cotta H, Fanghänel J (1993): Biomechanische Prinzipien in Diarthrosen und Synarthrosen. Teil III: Mechanik des Tibiofemoralgelenkes und Rolle der Kreuzbänder. Z Orthop Ihre Grenzgeb 131, 385-396

Nomura E, Horiuchi Y, Kihara M (2000): Medial patellofemoral ligament restraint in lateral patellar translation and reconstruction. The Knee $\underline{2}(7), 121-127$

Pankarter F, Betzler M, Eibelshäuser E, Merkle N (1979): Ergebnisse nach operativer Behandlung von Patellafrakturen. Unfallchir $\underline{5}, 19-24$

Petersson IF (1996): Occurrence of osteoarthritis of the peripheral joints in European populations. Ann Rheum Dis $\underline{55}$, 659-661

Pförringer W, Gorschewsky O: Die Patella aus orthopädischer und sportmedizinischer Sicht, Schattauer Verlag Stuttgart 2005

Powers CM (1998): Rehabilitation of patellofemoral joint disorders: a critical review. J Orthop Sports Phys Ther $\underline{28}$, 345-354

Rees D, Thompson SK (1985): Refracture of the patella. Injury $\underline{16}, 559-563$

Reynaud P, Donell S, Dejour D, Neyret PH (1996): Patellar fractures: pseudarthroses and malunions. Curr Opin Orthop $\underline{7}, 87-93$

Roaas A, Andersson GBJ (1982): Normal range of motion of the hip, knee and ankle joints in male subjects, 30 - 40 years of age. Acta Orthop Scand $\underline{53}, 205-208$ 
Rogge D, Oestern HJ, Gosse F (1985): Die Patellafraktur. Therapie und Ergebnisse. Der Orthopäde $\underline{14}$, 266-280

Rüter A, Trentz O, Wagner M: Unfallchirurgie, Kapitel Patella. 3. Auflage, Urban \& Schwarzenberg, München Wien Baltimore 2008

Schlenzka D, Schwesinger G, Plewka F (1979): Zur Entwicklung der Patella. Beitr Orthop Traumatol $\underline{26}, 296$

Scapinelli R (1967): Blood supply of the human patella: It's relation to ischaemic Necrosis after Fracture. J Bone Joint Surg Br $\underline{49-B}, 563-570$

Seedhom BB, Hargreaves DJ (1979): Transmission of the load in a knee joint with special reference to the role of the menisci. Eng Med $\underline{8}, 220-228$

Smirk C, Morris H (2003) The anatomy and reconstruction of the medial patellofemoral ligament. Knee $\underline{3}(10): 221-227$

Speck M, Regazzoni P (1994): Classification of patellar fractures. Z Unfallchir Versicherungsmed $\underline{87}, 27-30$

Sperner G, Wanitschek P (1989): Therapieformen und Behandlungsergebnisse der Patellafraktur. Unfallchirurgie $\underline{15}, 247-252$

Steensen RN, Dopirak RM, McDonald III WG(2004): The anatomy and isometry of the medial patellofemoral ligament: implications for reconstruction. Am J Sports Med $\underline{6}(32)$, 1509-1513

Stucki G, Meier D, Stucki S, Michael BA, Tyndall AG, Dick W, Theiler R (1996): Evaluation of a German version of WOMAC (Western Ontario and McMaster Universities) Arthrosis Index. Z Rheumatol 55(1), 40-49

Stürmer KM : Leitlinien Unfallchirurgie Thieme Verlag Stuttgart - New York 1999

Tegner Y, Lysholm J (1985): Rating Systems in the Evaluation of Knee Ligament Injuries. Clin Orthop $\underline{198}, 43-49$ 
Theodorou DJ, Theodorou SJ, Farooki S, Kakitsubata Y, Resnick D (2001): Osteonecrosis of the patella: imaging features. Clin Imaging 25(1), 60-65

Thomsen M, Mannel H, Spiering S, Dathe H, Kubein-Meesenburg D, Nägerl H (2002): Zur Biomechanik des Tibiofemoralgelenks und deren Umsetzung bei Knieorthesen. Der Orthopäde $\underline{31}, 914-920$

Tscherne H, Szyszkowitz R (1968): Die stabile Osteosynthese bei Kniescheibenbrüchen. Zbl Chir $\underline{93}, 1276$

Vicenzi G, Ruggieri N, Moroni A, Bettelli G, Innao V (1985): Multi-fragmented fractures of the patella: osteosynthesis or partial patellectomy? Chir Organi Mov $\underline{70}, 133-140$

Weinberg AM, Tscherne H: Unfallchirurgie im Kindesalter: 1. Allgemeiner Teil, Kopf, Obere Extremität, Wirbelsäule 2. Untere Extremität, Körperhöhlen, Besonderheiten des kindlichen Skelette, 1. Auflage; Springer-Verlag, Berlin Heidelberg New York 2006

Wendl K, Zinser R, Hochstein P, Grützner PA (2002): Frakturen der Kniescheibe - Eine häufig unterschätzte Verletzung. Trauma Berufkrankh $\underline{4}, 30-37$

Wiberg G (1941): Roentgenographic and anamotic studies on the femoropatellar joint: With special reference to chondromalacia patellae. Acta Orthop Scand 12, 319-340

Wild M, Khayel T, Miersch D, Windolf J, Hakimi M (2008) : Die Zuggurtungsosteosynthese nach Patellafraktur. Unfallchirurg 111, $892-897$

Wild M, Windolf J, Flohé S (2010): Patellafrakturen. Unfallchirurg 113, 401-412

Wirth CJ, Zichner L, Kohn D: Orthopädie und Orthopädische Chirurgie, Thieme Verlag Stuttgart 2005

Wismans J, Veldpaus F, Janssen J, Huson A (1980): A threedimensional mathematical model of the knee-joint. J Biomech $\underline{13}, 677-685$

Zuppinger $\mathrm{H}$ : Die aktive Flexion im unbelasteten Kniegelenk (Züricher med. Habilitationsschrift). Bergmann, Wiesbaden 1904 
8. Anhang: Nachuntersuchungsbogen, Scores

\section{Klinischer Nachuntersuchungsbogen}

Datum:

Patientennummer:

\section{Visuell numerische Analogskala Schmerz (VASS)}

Markieren Sie mit einem Strich, wie stark Sie den Schmerz durchschnittlich in den letzten Tagen in Ihrem operierten Gelenk empfinden.

0 steht für keinen Schmerz und

10 für den maximal vorstellbaren Schmerz 


\section{American Knee Society Score (AKSS)}

A Kniescore rechts: links:

I. Schmerzen:

Kein Schmerz.

leicht oder gelegentlich.

nur beim Treppensteigen.

beim Gehen und Treppensteigen

mäßiger Schmerz

gelegentlich

ständig

starker Schmerz

II. Extensio/Flexion:

1 Punkt pro $5^{\circ}$, max. 25 Punkte.

III. Stabilität:

anterior-posterior

$<5 \mathrm{~mm}$

5-10 $\mathrm{mm}$

$>10 \mathrm{~mm}$

medio-lateral

$<5^{\circ}$.

$6^{\circ}-9^{\circ}$

$10^{\circ}-14^{\circ}$

$>15^{\circ}$.

IV. Abzüge:

Beugekontraktur

$$
<5^{\circ} \text {. }
$$

$5^{\circ}-10^{\circ}$

$11^{\circ}-15^{\circ}$

$16^{\circ}-20^{\circ}$

$>20^{\circ}$

Streckdefizit

kein.

unter $10^{\circ}$

$10^{\circ}-20^{\circ}$

über $20^{\circ}$

Valguseinstellung

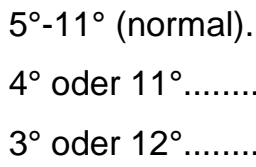




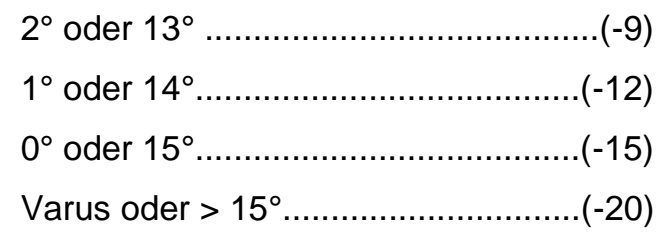

\section{B Funktionsscore}

I. Gehstrecke:

unbegrenzt............................................................ (50)

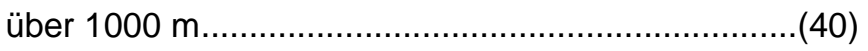

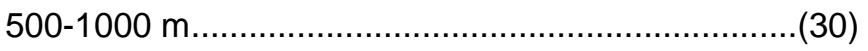

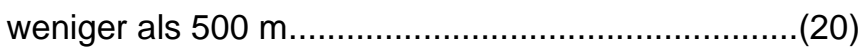

hausgebunden........................................................ (10)

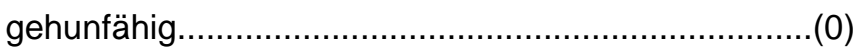

II. Treppensteigen:

normal hoch und herunter.....................................(50)

normal hoch, herunter mit Geländer.........................(40)

hoch und herunter mit Geländer..............................(30)

hoch mit Geländer, herunter unmöglich....................(15)

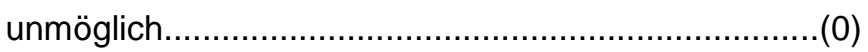

III. Abzüge:

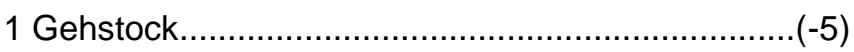

2 Gehstöcke............................................................ $(-10)$

Gehstützen oder Gehwagen...................................(-20) 


\section{Tegner-Aktivitätsscore}

\section{Wettkampf- bzw. Leistungssport}

Fussball, nationales oder internationales Niveau

\section{Wettkampfsport}

Fussball, Niedrige Ligen, Eishockey, Ringen, Gymnastik

\section{Wettkampfsport}

Squash, Badminton, Leichtathletik (Sprungsportarten), Abfahrtski

\section{Wettkampfsport}

Tennis, Leichtathletik (Laufsportarten), Handball, Basketball, Motorrad

\section{Freizeitsport}

Fussball, Eishockey, Squash, Leichathletik (Sprungsportarten),

Orientierungslauf, Crosslauf

\section{Freizeitsport}

Tennis, Badminton, Handball, Basketball, Abfahrtsski, Jogging (mind. 5 mal wöchentlich)

\section{Arbeit}

Schwere Arbeit (z.B. Bauarbeiter)

Wettkampf- und Freizeitsport

Radfahren, Skilanglauf

\section{Freizeitsport}

Jogging auf unebenen Untergrund (mind. 2 mal wöchentlich)

\section{Arbeit}

Mittelschwere Arbeit (z.B. Fernfahrer)

\section{Freizeitsport}

Radfahren, Skilanglauf, Jogging auf ebenem Untergrund (mind. 2 mal wöchentlich) 
3 Arbeit

Leichte Arbeit (z.B. Krankenschwester)

Wettkampf- und Freizeitsport

Schwimmen

Gehen im Wald möglich

2 Arbeit

Leichte Arbeit

Gehen auf unebenem Grund möglich, aber Gehen im Wald unmöglich

1 Arbeit

Sitzende Tätigkeit

Gehen auf ebenem Untergrund möglich

0 Bettlägerigkeit oder Invalidität, Rente wegen Knieproblemen oder Sprunggelenksproblemen 


\section{WOMAC-Score}

Fragebogen zu den Beschwerden im betroffenen Gelenk

Sie werden gebeten, nach diesem Muster die Stärke Ihrer Schmerzen, Ihre Steifigkeit oder Behinderung anzugeben. Bitte vergessen sie nicht, je mehr rechts Sie das „X“ ankreuzen, umso mehr Schmerzen, Steifigkeit oder Behinderung haben Sie.

\section{A Schmerzfragen}

Die folgenden Fragen beziehen sich auf die Stärke Ihrer Schmerzen, die Sie im kranken Gelenk haben. Bitte geben Sie für jede Frage die Stärke der Schmerzen an, die Sie in den letzten 2 Tagen verspürt haben. Bitte kreuzen Sie die zutreffenden Kästchen an.)

\section{Wie starke Schmerzen haben Sie beim}

\section{Gehen auf ebenem Boden}

Keine

Schmerzen

\begin{tabular}{|l|l|l|l|l|l|l|l|l|l|l|}
\hline & & & & & & & & & & \\
\hline
\end{tabular}

extreme Schmerzen

\section{Treppen hinauf- oder hinuntersteigen}

Keine

Schmerzen

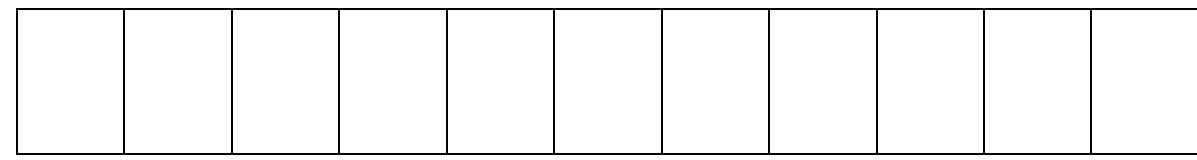

extreme Schmerzen

\section{Nacht im Bett}

Keine

Schmerzen

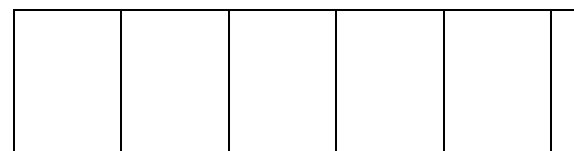

extreme Schmerzen

\section{Sitzen oder Liegen}

Keine

Schmerzen

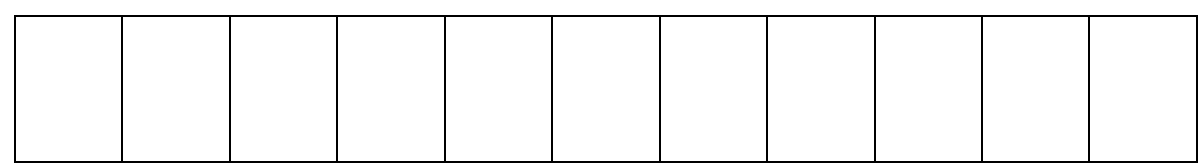

extreme

Schmerzen 
5. Aufrecht stehen

Keine

Schmerzen

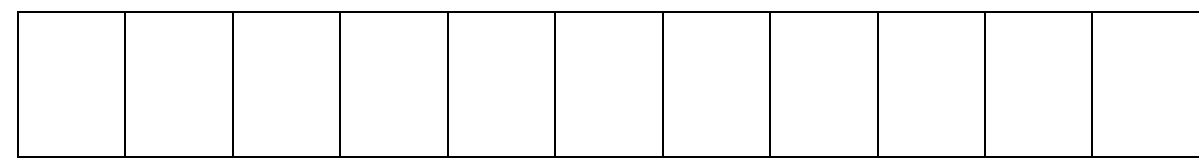

extreme

Schmerzen

\section{B Fragen zur Steifigkeit}

Die folgenden Fragen beziehen sich auf die Steifigkeit (nicht die Schmerzen) Ihres kranken Gelenkes. Steifigkeit ist ein Gefühl von Einschränkung oder Langsamkeit in der Beweglichkeit, wenn Sie Ihre Gelenke bewegen. Bitte geben Sie für jede Frage die Stärke der Steifigkeit an, die Sie in den letzten 2 Tagen verspürt haben. (Bitte kreuzen Sie die zutreffenden Kästchen an).

1. Wie stark ist die Steifigkeit gerade nach dem Erwachen am Morgen? Keine Steifigkeit

\begin{tabular}{|l|l|l|l|l|l|l|l|l|l|l|}
\hline & & & & & & & & & & \\
\hline
\end{tabular}

extreme Steifigkeit

2. Wie stark ist Ihre Steifigkeit nach Sitzen, Liegen oder Ausruhen im späteren Verlauf des Tages?

Keine

Steifigkeit

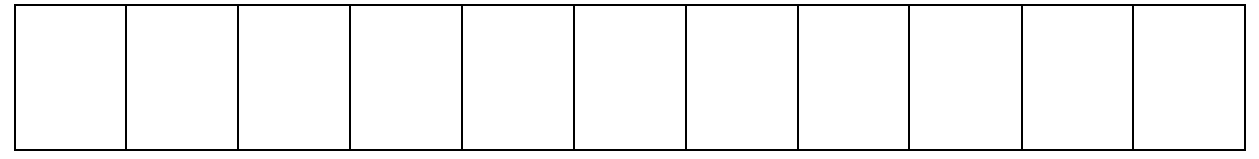

extreme Steifigkeit

\section{Fragen zur körperlichen Tätigkeit}

Die folgenden Fragen beziehen sich auf Ihre körperliche Tätigkeit. Damit ist Ihre Fähigkeit gemeint, sich im Altag zu bewegen und um sich selbst zu kümmern. Bitte geben Sie für jede der folgenden Aktivitäten den Schwierigkeitsgrad an, den Sie in den letzten 2 Tagen wegen Beschwerden in Ihrem kranken Gelenk gespürt haben. (Bitte kreuzen Sie die zutreffenden Kästchen an.)

Wie groß ist ihre Schwierigkeit beim:

\section{Treppen hinuntersteigen}

Keine

Schwierigkeiten

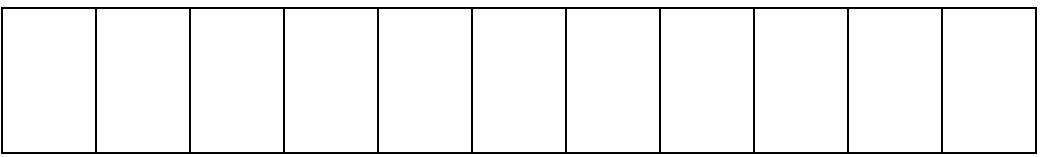

extreme

Schwierigkeiten 
2. Treppen hinaufsteigen

Keine

Schwierigkeiten

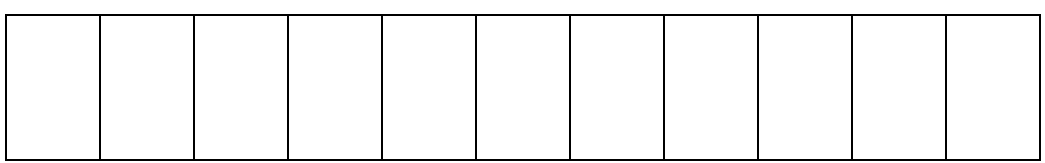

extreme

Schwierigkeiten

\section{Aufstehen vom Sitzen}

Keine

Schwierigkeiten

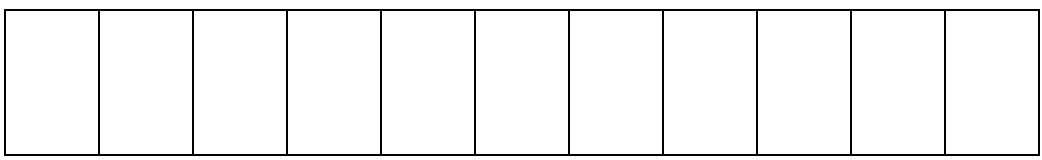

extreme

Schwierigkeiten

\section{Stehen}

Keine

Schwierigkeiten

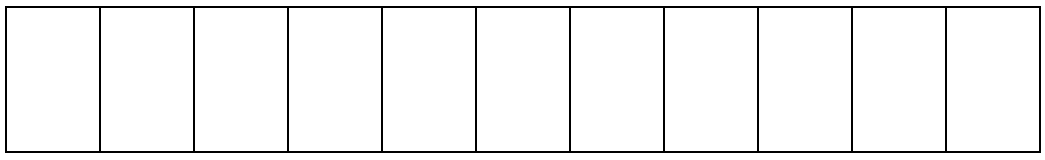

extreme

Schwierigkeiten

\section{Sich zum Boden bücken}

Keine

Schwierigkeiten

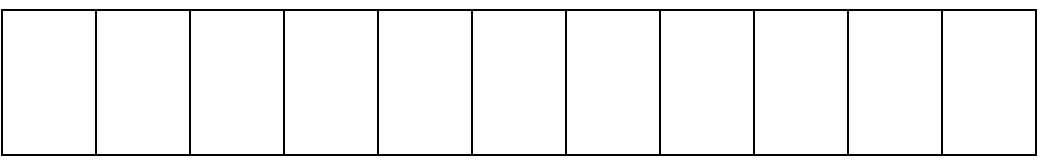

extreme

Schwierigkeiten

6. Gehen auf ebenem Boden

Keine

Schwierigkeiten

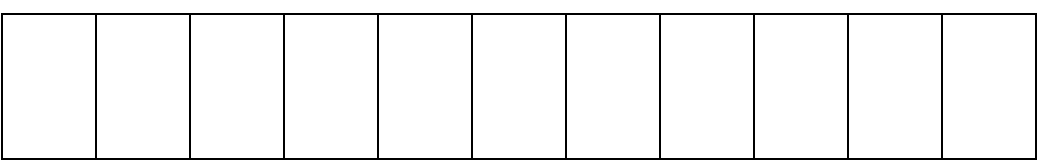

extreme

Schwierigkeiten

7. Einsteigen ins Auto/Aussteigen aus dem Auto

Keine

Schwierigkeiten

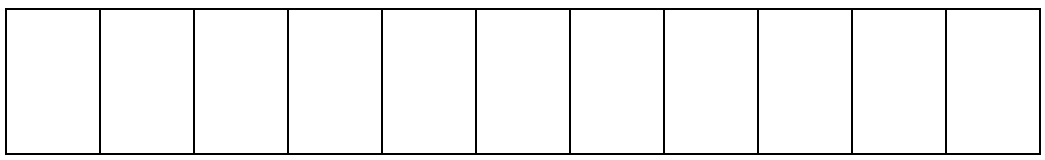

extreme

Schwierigkeiten

\section{Einkaufen gehen}

Keine

Schwierigkeiten

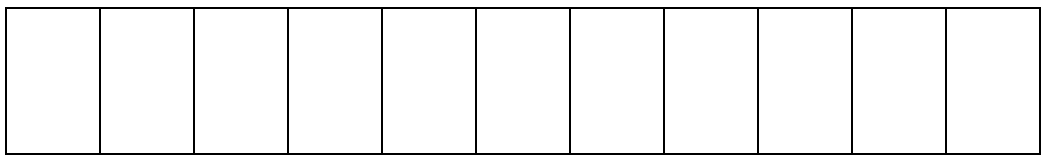

extreme

Schwierigkeiten

9. Socken/Strümpfe anziehen

Keine

Schwierigkeiten

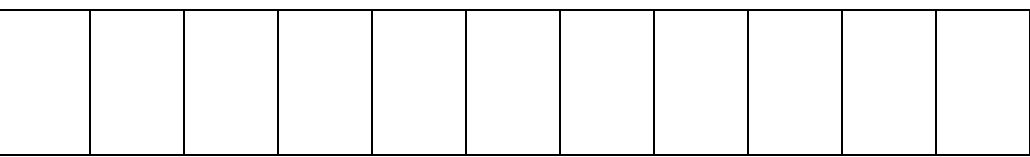

extreme

Schwierigkeiten 
10. Aufstehen vom Bett

Keine

Schwierigkeiten

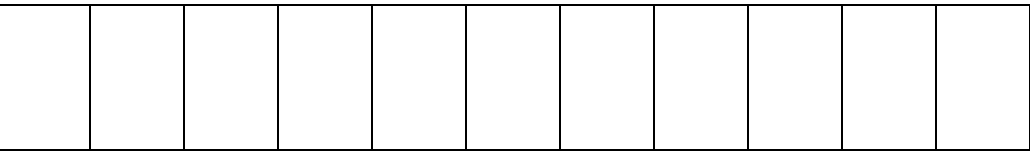

extreme

Schwierigkeiten

\section{Socken/Strümpfe ausziehen}

Keine

Schwierigkeiten

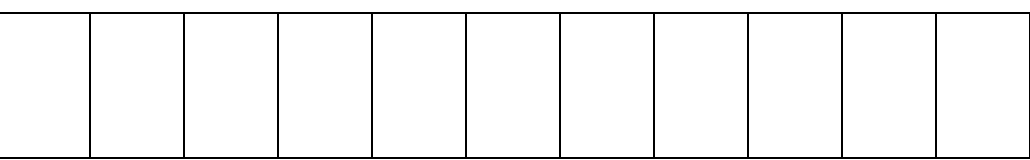

extreme

Schwierigkeiten

\section{Liegen im Bett}

Keine

Schwierigkeiten

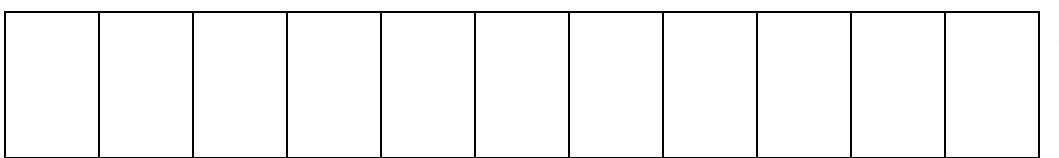

extreme

Schwierigkeiten

\section{Ins Bad/aus dem Bad steigen}

Keine

Schwierigkeiten

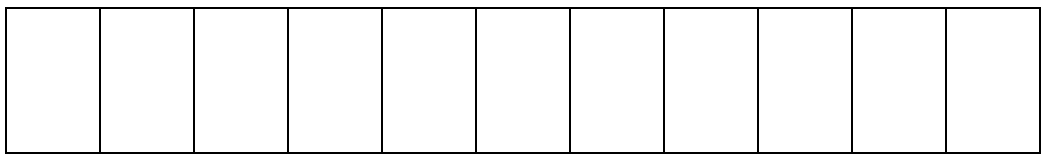

extreme

Schwierigkeiten

\section{Sitzen}

Keine

Schwierigkeiten

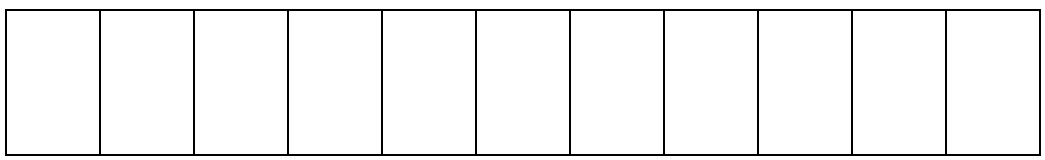

extreme

Schwierigkeiten

\section{Sich auf die Toilette setzen/Aufstehen von der Toilette}

Keine

Schwierigkeiten

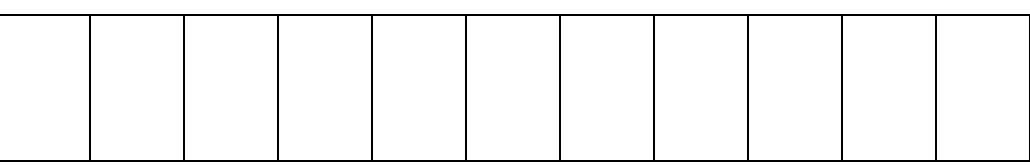

extreme

Schwierigkeiten

\section{Anstrengenden Hausarbeiten}

Keine

Schwierigkeiten

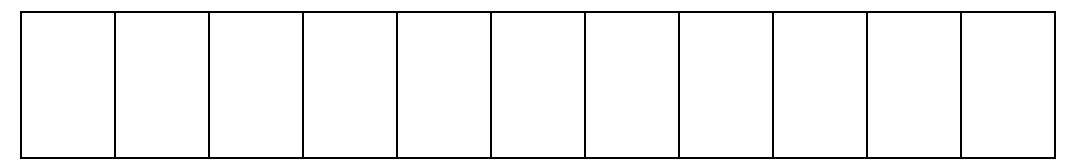

extreme

Schwierigkeiten

\section{Leichte Hausarbeiten}

Keine

Schwierigkeiten

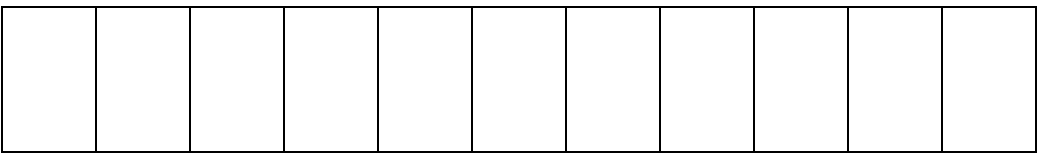

extreme

Schwierigkeiten 


\section{Lysholm-Score:}

\begin{tabular}{|l|l|r|}
\hline Hinken & Nein & $\mathbf{5}$ \\
\hline & Wenig oder zeitweise & $\mathbf{3}$ \\
\hline & Stark oder immer & $\mathbf{1}$ \\
\hline
\end{tabular}

\begin{tabular}{|l|l|l|}
\hline Belastung & Vollbelastung & $\mathbf{5}$ \\
\hline & Gehstützen oder Stock & $\mathbf{3}$ \\
\hline & Belastung nicht möglich & $\mathbf{0}$ \\
\hline
\end{tabular}

\begin{tabular}{|l|l|r|}
\hline Blockierung & Keine Blockierung und kein Gefühl der Einklemmung & $\mathbf{1 5}$ \\
\hline & Gefühl der Einklemmung aber keine Blockierung & $\mathbf{1 0}$ \\
\hline & Gelegentliche Blockierung & $\mathbf{6}$ \\
\hline & Häufige Blockierung & $\mathbf{2}$ \\
\hline & Blockiertes Gelenk bei Untersuchung & $\mathbf{0}$ \\
\hline
\end{tabular}

\begin{tabular}{|l|l|c|}
\hline Instabilität & Niemals „giving way“ Phänomen & $\mathbf{2 5}$ \\
\hline & $\begin{array}{l}\text { "giving way" selten während des Sports oder anderer } \\
\text { schwerer Anstrengung }\end{array}$ & $\mathbf{2 0}$ \\
& $\begin{array}{l}\text { "giving way" häufigwährend des Sports oder anderer } \\
\text { schwerer Anstrengung (oder unmöglich, daran } \\
\text { teilzunehmen) }\end{array}$ & $\mathbf{1 5}$ \\
\hline & $\begin{array}{l}\text { "giving way" gelegentlich während Tätigkeiten des } \\
\text { Alltags }\end{array}$ & $\mathbf{1 0}$ \\
\hline & "giving way" oft während Tätigkeiten des Alltags & $\mathbf{5}$ \\
\hline & "giving way" bei jedem Schritt & $\mathbf{0}$ \\
\hline
\end{tabular}

\begin{tabular}{|l|l|c|}
\hline Schmerzen & Keine & 25 \\
\hline & $\begin{array}{l}\text { unregelmäßig and gering während schwerer } \\
\text { Anstrengung }\end{array}$ & 20 \\
\hline & deutlich/ausgeprägt während schwerer Anstrengung & $\mathbf{1 5}$ \\
\hline & $\begin{array}{l}\text { Deutlich während oder nach dem Gehen von mehr als 2 } \\
\mathrm{km}\end{array}$ & $\mathbf{1 0}$ \\
\hline
\end{tabular}




\begin{tabular}{|l|l|r|}
\hline & $\begin{array}{l}\text { Deutlich während oder nach dem Gehen von weniger } \\
\text { als 2 km }\end{array}$ & $\mathbf{5}$ \\
\hline ständig & $\mathbf{0}$ \\
\hline
\end{tabular}

\begin{tabular}{|l|l|r|}
\hline Schwellung & Keine & $\mathbf{1 0}$ \\
\hline & Bei schwerer Anstrengung & $\mathbf{6}$ \\
\hline & Bei gewöhnlicher Anstrengung & $\mathbf{2}$ \\
\hline & ständig & $\mathbf{0}$ \\
\hline
\end{tabular}

\begin{tabular}{|l|l|r|}
\hline Treppensteigen & Kein Problem & $\mathbf{1 0}$ \\
\hline & Ein wenig beeinträchtigt & $\mathbf{6}$ \\
\hline & Schritt für Schritt & $\mathbf{2}$ \\
\hline & Nicht möglich & $\mathbf{0}$ \\
\hline
\end{tabular}

\begin{tabular}{|l|l|r|}
\hline Hocken & Kein Problem & $\mathbf{5}$ \\
\hline & Wenig beeinträchtigt & $\mathbf{4}$ \\
\hline & Nicht über $90^{\circ}$ & $\mathbf{2}$ \\
\hline & Nicht möglich & $\mathbf{0}$ \\
\hline
\end{tabular}

\section{Patientenzufriedenheit}

Markieren Sie mit einem vertikalen Strich, wie sehr Sie mit dem operativen Ergebnis zufrieden sind

0 steht für „absolut unzufrieden“ und

10 für „vollkommen zufrieden“ mit dem Resultat 